\title{
FLUID-STRUCTURE INTERACTION OF A VARIABLE CAMBER COMPLIANT WING
}

\author{
Thesis \\ Submitted to \\ The School of Engineering of the \\ UNIVERSITY OF DAYTON \\ In Partial Fulfillment of the Requirements for \\ The Degree of \\ Master of Science in Aerospace Engineering \\ By \\ Samuel C. Miller \\ UNIVERSITY OF DAYTON \\ Dayton, Ohio
}

May, 2015 


\section{FLUID-STRUCTURE INTERACTION \\ OF A VARIABLE CAMBER COMPLIANT WING}

Name: Miller, Samuel C.

\section{APPROVED BY:}

Markus P. Rumpfkeil, Ph.D.

Advisor Committee Chairman

Assistant Professor, Department of

Mechanical and Aerospace Engineering
James J. Joo, Ph.D.

Committee Member

Adjunct Professor, Department of

Mechanical and Aerospace Engineering

José A. Camberos, P.E., Ph.D

Committee Member

Adjunct Professor, Department of

Mechanical and Aerospace Engineering

John G. Weber, Ph.D.

Associate Dean

School of Engineering
Eddy Rojas, Ph.D., M.A., P.E.

Dean, School of Engineering 


\section{ABSTRACT \\ FLUID-STRUCTURE INTERACTION \\ OF A VARIABLE CAMBER COMPLIANT WING}

Name: Miller, Samuel C.

University of Dayton

Advisor: Dr. Markus P. Rumpfkeil

This thesis presents results from loosely-coupled fluid-structure interaction (FSI) simulations of a flexible wing which used FUN3D to compute the aerodynamic flow-fields and Abaqus to calculate the structural deformations. NASA Langley also provides a general 3D algorithm to interpolate between dissimilar meshes which is used here to map pressures and displacements between the aerodynamic and structural codes. This method is applied to the AFRL - developed "Variable Camber Compliant Wing" (VCCW), which is an adaptable wing designed to target airfoil shapes between a NACA 2410 and 8410. The VCCW was tested in the Vertical Wind Tunnel facility at WrightPatterson Air Force Base, which provided experimental data in the form of static pressure tap data, digital image correlation, and oil flow visualization. The combined solutions from Abaqus, FUN3D, and mesh interpolation solvers created FSI results that were similar in trend to the experiment, but consistently under-predicted the deformations of the $\mathrm{VCCW}$, due to the differences between the experiment and simulation, including the choice of material models and the assumption of ideal conditions. This thesis has been cleared by the Wright-Patterson AFB Public Affairs Office, case number 88ABW-2015-1502. This provides proof of public release, distribution unlimited. 
For Bethany, Oliver, and Clara 


\section{ACKNOWLEDGMENTS}

First and foremost, I want to thank God for the blessing and provision to pursue a master's degree in Aerospace Engineering. I would also like to thank the staff and leadership of the Aerospace Vehicles Division in the Aerospace Systems Directorate of the Air Force Research Laboratory at Wright-Patterson AFB, Dayton, OH for allowing me to be part of the Variable Camber Compliant Wing (VCCW) project. Specifically, Dr. James Joo, for his leadership of the VCCW project, and Dr. Chris Marks, Ms. Lauren Zientarski, and Dr. Adam Culler, who formed the core of the VCCW team. I would also like to thank Drs. John Benek, Charles Tyler, and Ryan Durscher for their support and assistance with all things CFD and FUN3D-related, and Dan Galbraith for his help with learning and writing Python. I want to thank Jon Geiger, Oliver Leembruggen, Brian Smyers, and the staff at the Vertical Wind Tunnel facility for providing the experimental data. In addition, I want to acknowledge and thank my advisor, Dr. Markus Rumpfkeil, for his support throughout the entirety of this thesis and helping me through the process. And, last but not least, I especially want to thank my wife, Bethany, and our children, Oliver and Clara, for their love and support through all of the long hours I devoted to this project. Without them, and the combined effort of all those involved, this thesis would not have been possible. 


\section{TABLE OF CONTENTS}

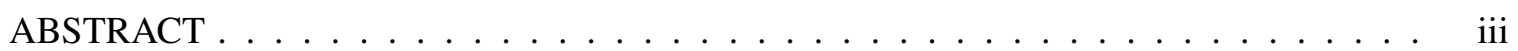

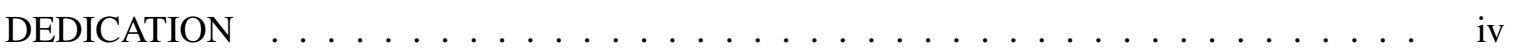

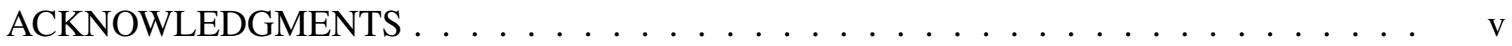

LIST OF FIGURES $\ldots \ldots \ldots \ldots \ldots \ldots \ldots \ldots \ldots \ldots \ldots$ viii

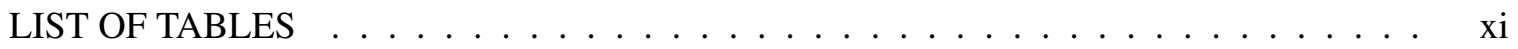

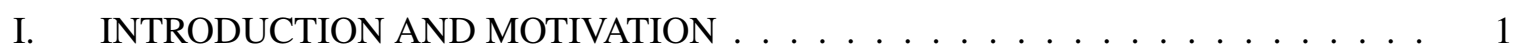

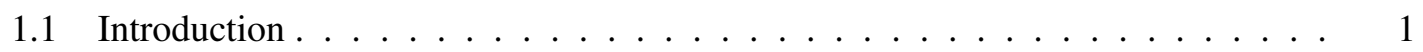

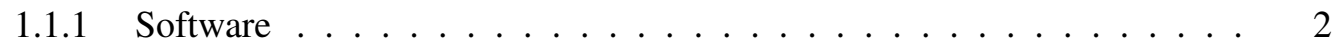

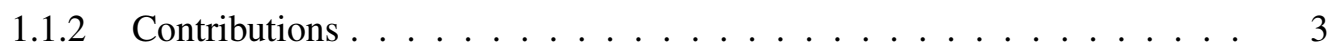

II. FLUID-STRUCTURE INTERACTION $\ldots \ldots \ldots \ldots \ldots$

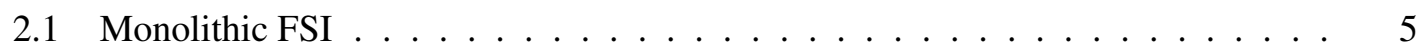

2.2 Partitioned FSI Coupling . . . . . . . . . . . . . . . . . 6

2.2.1 Partitioned Coupling Methods . . . . . . . . . . . . . . . 7

III. VARIABLE CAMBER COMPLIANT WING . . . . . . . . . . . . . . . . 10

3.1 Wind Tunnel Testing . . . . . . . . . . . . . . . . . . . . . . . . . . . . . . .

3.2 Digital Image Correlation . . . . . . . . . . . . . . . . . . . . 13

3.3 Optical Scans . . . . . . . . . . . . . . . . . . . . . 15

3.4 Oil Flow Visualization . . . . . . . . . . . . . . . . . . . . 17

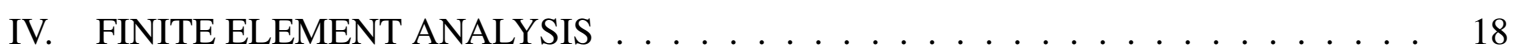

4.1 Material Modeling . . . . . . . . . . . . . . . . . . . 20

4.2 Compliant Mechanism Actuation _ . . . . . . . . . . . . . . 22

4.3 NACA Profiles - FSI versus Experiment . . . . . . . . . . . . . . 23 
4.4 FEA Runtime Statistics . . . . . . . . . . . . . . . . . . . . . . . . . . . 24

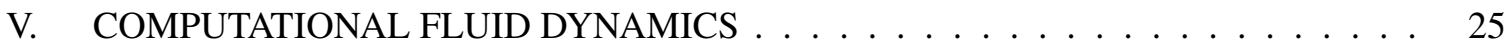

5.1 FUN3D - External Aeroelastic Mode . . . . . . . . . . . . . . . . . . . 27

5.1.1 Support Structure Interference Effects . . . . . . . . . . . . . . . . . . 29

5.2 Scanned versus Ideal Geometry $\ldots \ldots \ldots \ldots$

VI. MESH INTERPOLATION $\ldots \ldots \ldots \ldots \ldots \ldots \ldots$

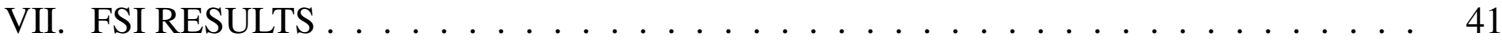

7.1 DIC Comparison . . . . . . . . . . . . . . . . . . . . . 44

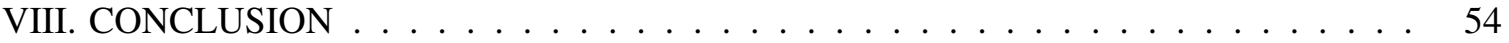

8.1 Future Work . . . . . . . . . . . . . . . . . . 56

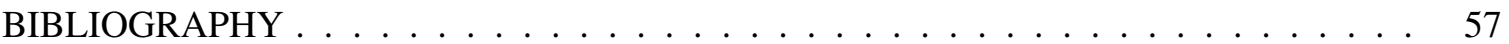
APPENDICES:

A. Additional CFD Results . . . . . . . . . . . . . . . . . . . . . . . . . 59

B. Additional DIC Results . . . . . . . . . . . . . . . . . . . . . . . . . . 67 


\section{LIST OF FIGURES}

2.1 Loosely-Coupled, Partitioned Solution Algorithm . . . . . . . . . . . . . . . . . 9

$3.16 \mathrm{ft}$ VCCW model installed in the WPAFB Vertical Wind Tunnel . . . . . . . . . 12

3.2 Raw DIC data surfaces for 4 cambers at $0^{\circ}$ angle of attack $\ldots \ldots \ldots \ldots$

3.3 Sample measured profiles specifying maximum camber and local twist . . . . . . 16

3.4 Local twist effects on camber and coefficient of lift f . . . . . . . . . . . 16

3.5 Oil Flow for NACA 2410 and 8410 at $8^{\circ} \ldots \ldots \ldots \ldots \ldots$

4.1 Abaqus FEA mesh configuration showing displacements colored by magnitude . . 19

4.2 Leading Edge Material Estimate - Behavior Comparison _ . . . . . . . . . . . 21

4.3 Material Test Data Specimens f . . . . . . . . . . . . . . . . 22

4.4 NACA 2410 Profile Comparisons _ . . . . . . . . . . . . . . . . . . . . 23

4.5 NACA 4410 Profile Comparisons _ . . . . . . . . . . . . . . . . . . . . . . . 24

5.1 CFD mesh configuration (slice at 50 percent span) $\ldots \ldots \ldots$

5.2 CFD Mesh Resolution Study: Initial FSI configuration (NACA 2410) $\ldots \ldots \ldots$

5.3 CFD convergence history for the NACA 4410 (top) and 2410 (bottom) cambers at

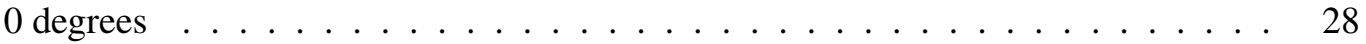

5.4 Lift and drag comparison for support structure interference . . . . . . . . . . . 29

5.5 Support Structure Interference $\ldots \ldots \ldots \ldots \ldots$. . . . . . . . . . . . 31 
5.6 NACA 3410 Profile Comparison $\ldots \ldots \ldots \ldots \ldots$

5.7 Differences in the mesh topology between scan and ideal geometries . . . . . . . 33

5.8 Lift and Drag Comparisons - Scanned vs Ideal . . . . . . . . . . . . . . . . . 34

5.9 Scanned and Ideal Total Lift vs Experimental Sectional Lift . . . . . . . . . . . . . 34

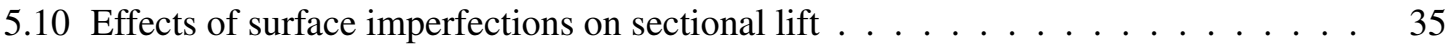

5.11 Iso-surfaces of Reversed Flow and Stagnation $\ldots \ldots \ldots$

5.12 Surface Pressure Distribution . . . . . . . . . . . . . . . . 36

5.13 Leading edge shape variation between ideal and scanned geometry at the same spanwise location. Colored by Mach number . . . . . . . . . . . . . . . . . . . 37

6.1 Leading Edge Interpolation Error . . . . . . . . . . . . . . . . . . . . . . 39

7.1 FSI convergence history for the NACA 2410 and NACA $4410 \ldots \ldots$. . . . . 43

7.2 Deformation and Camber Progression . . . . . . . . . . . . . . . . . . 44

7.3 FSI Trailing Edge Deflection Progression $\ldots \ldots \ldots \ldots \ldots$

7.4 FSI vs. DIC Results - Displacement Slices . . . . . . . . . . . . . . . . . . . . 47

$7.5 \mathrm{CFD} /$ FEA configuration effects $\ldots \ldots \ldots \ldots \ldots \ldots \ldots$

7.6 NACA 2410 Target Camber, Alpha $=0^{\circ} \ldots \ldots \ldots \ldots \ldots$

7.7 NACA 4410 Target Camber, Alpha $=0^{\circ} \ldots \ldots \ldots \ldots \ldots$

7.8 NACA 4410 Target Camber, Alpha $=-4^{\circ} \ldots \ldots \ldots \ldots \ldots$

A1.1 CFD Mesh Resolution Study: Initial FSI configuration (NACA 2410) $\ldots \ldots \ldots$

A1.2 $-6^{\circ}$ Support Structure Interference $\ldots \ldots \ldots \ldots$. . . . . . . . . 61

A1.3 $-4^{\circ}$ Support Structure Interference $\ldots \ldots \ldots \ldots$. . . . . . . . . . . 62

A1.4 $-2^{\circ}$ Support Structure Interference $\ldots \ldots \ldots \ldots$ 
A1.5 $0^{\circ}$ Support Structure Interference . . . . . . . . . . . . . . . . . . . . . . . 64

A1.6 $2^{\circ}$ Support Structure Interference $\ldots \ldots \ldots \ldots$. . . . . . . . . . 65

A1.7 $4^{\circ}$ Support Structure Interference . . . . . . . . . . . . . . . . . . . . 66

B2.1 Camber Progression for $-4^{\circ} \ldots \ldots \ldots \ldots \ldots \ldots \ldots$

B2.2 NACA 4410, Alpha $=0$, Span-wise slice at spar $\ldots \ldots \ldots \ldots$

B2.3 NACA 2410 Target Camber: $-4^{\circ} \ldots \ldots \ldots \ldots \ldots \ldots$

B2.4 NACA 2410 Target Camber: $6^{\circ} \ldots \ldots \ldots \ldots \ldots \ldots \ldots$

B2.5 NACA 4410 Target Camber: $6^{\circ} \ldots \ldots \ldots \ldots \ldots \ldots$ 


\section{LIST OF TABLES}

2.1 Conventional Serial Staggered Scheme . . . . . . . . . . . . . . . . . 8

3.1 Experimental Test Configuration $\ldots \ldots \ldots \ldots \ldots \ldots \ldots$

7.1 FSI Analysis Matrix . . . . . . . . . . . . . . . . . . . . . . 41 


\section{CHAPTER I}

\section{INTRODUCTION AND MOTIVATION}

\subsection{Introduction}

Fluid-structure interaction (FSI) can be defined as the phenomena that occurs at the interface between a structure and the surrounding fluid. Examples can be found in biological flows such as blood flow in arteries or airflow in lungs, aeroelastic effects in aircraft, and architectural responses to wind forces. The study of FSI is of particular importance for aerospace applications where aerodynamic forces cause structural motion, ranging from aeroelastic flutter to significant and sustained deformation. Oscillatory effects such as flutter can cause serious structural fatigue and a lack of adequate stability and control power. In the past, aeroelastics have been an afterthought when applied to aircraft design, but as the aerospace industry moves towards an increased use of composites, this will greatly increase the need to study the interaction between flexible structures and their aerodynamics during the design process. Incorporating FSI analysis into the design process will facilitate this development and eventually help to improve design process efficiency and optimize the design itself.

In order to study the challenges and advantages that flexible aircraft designs can present, the Air Force Research Laboratory (AFRL) has developed a novel adaptive Variable Camber Compliant Wing (VCCW) that can actively change camber by using a compliant mechanism [1]. The goal of the design is to create a distributed camber control capable of reconfiguration through continuous 
deformations to optimize its shape to suit its current altitude, airspeed, and lift-to-drag (L/D) ratio requirements. Detrimental aerodynamic conditions such as separated flow and parasitic drag, caused by gaps and holes from traditional control surfaces, are avoided by the seamless construction of the VCCW. Due to the significant coupling between the structural and aerodynamic domains unique to its design, the VCCW provided the perfect test case for a fluid-structure interaction analysis.

The simulation of fluid-structure interaction applied to the VCCW was accomplished through a partitioned loosely-coupled approach. This approach coupled finite element analysis (FEA) and computational fluid dynamics (CFD), two separate computational physics software suites. The FEA software solved for structural deformations due to actuation and applied pressure loads and the CFD software solved for the pressure loads as response to the aerodynamic flow over the wing. This project presents the methodology used to couple separate software codes for an FSI analysis, how it was specifically applied to the Variable Camber Compliant Wing, and the results. Results from this thesis were also presented at AIAA Scitech 2015 in Kissimmee, FL [2]. Lessons learned for future FSI simulations are also presented.

\subsubsection{Software}

Coupling the two computational solvers was fairly straightforward from a computer science perspective, but the crucial step for a partitioned, loosely-coupled method is the interpolation of the separate solutions. CFD and FEA meshes typically have significantly different resolutions that require a careful mapping between them.

FUN3D [3], which is developed and maintained by the NASA Langley Research Center, was the CFD solver used. It is an unstructured, Reynolds-Averaged Navier-Stokes (RANS), finite volume CFD solver. FUN3D can support steady or time-dependent simulations, with moving, adaptable, and overset grids, design optimization, and adjoint-based error estimation. The external aeroelastic 
module built into FUN3D allowed boundary surfaces to be imported and exported into the volume mesh. At the conclusion of each CFD run, the boundary surface was exported in a format specifically designed to be sent to the interpolation code used to map between FEA and CFD.

Abaqus, a commercially available finite element analysis software suite, was used to create and analyze the structural response of the compliant wing under deformation due to actuation and aerodynamic loading. Abaqus includes Python modules which allowed detailed access to the structural model and results. Extensive scripting was used to parse the output, create displacement and model definition files, and pass the data to the mesh interpolation code.

Samareh $[4,5]$ provided a general 3D algorithm to facilitate transfers between different meshes in various formats. This software, Discrete Data Transfer Between Dissimilar Meshes (DDTBDM), handles the transfer of displacement outputs given by Abaqus onto the aeroelastic surface output given by FUN3D. This mapped CFD mesh was then sent back to FUN3D as a deformed surface.

Python was used extensively to manage communication between the three separate codes. It facilitated automatic monitoring of solutions, file management and format conversions, as well as local and remote server job submission. An object-oriented approach was taken to allow future applications to generic fluid-structure interaction problems beyond the application presented here.

\subsubsection{Contributions}

The work presented in this thesis is the result of a combined, widespread effort across the Air Vehicles Division in the Aerospace Systems Directorate of the Air Force Research Laboratory located at Wright-Patterson Air Force Base, Dayton, OH. The VCCW project was created and managed by Dr. James Joo, who pioneered the design of the prototype. Dr. Chris Marks, Dr. Adam Culler, and Lauren Zientarski, were instrumental in the design, FEA model creation, testing, and fabrication of the $6 \mathrm{ft}$ and $1 \mathrm{ft}$ VCCW prototypes. Jon Geiger, Oliver Leembruggen, Brian Smyers, Capt. Tim 
Cleaver, and the staff at the experimental testing branch, were responsible for the majority of the data generated by wind tunnel testing, digital image correlation, and surface oil flow visualization. The prototype and all of the experimental data is the result of their work, upon which the numerical FSI analysis presented in this thesis is built. 


\section{CHAPTER II}

\section{FLUID-STRUCTURE INTERACTION}

There are two different fundamental ways to conduct fluid-structure interaction analysis: Partitioned or monolithic. This distinction comes from the way that the fluid and structure equations are solved. Monolithic techniques solve the fluid and structure equations simultaneously whereas the partitioned approach solves each equation separately. Both coupling techniques have advantages and disadvantages depending on the particular problem and software availability.

\subsection{Monolithic FSI}

Monolithic schemes combine both the fluid and structure systems of equations which are then solved simultaneously. While this has the advantage of imposing the proper equilibrium at the FSI interface, it involves solving a massive set of nonlinear algebraic equations that are dissimilar in behavior. Fluid dynamics equations are typically cast in an Eulerian reference system, whereas structural equations are in the Lagrangian reference system. This dissimilarity causes the structural equations to become an order of magnitude stiffer compared to the fluid equations, which makes it very difficult to solve computationally [6]. Monolithic approaches are simpler to analyze from a mathematical point of view, but they do not take advantage of the major differences in the equations and the resulting solver architectures in the way that partitioned methods can. Attempts to solve these numerical problems include solution strategies for large-scale flow simulations [7], parallel 
mesh update strategies [8], and preconditioning techniques with rapid iterative solution methods [9]. Monolithic methods typically perform better than the partitioned approaches in cases where FSI is significant, e.g., biological flow. Because of the cost and complexity associated with large scale problems, monolithic methods are often limited to smaller academic problems or those in which the FSI is the dominant physical effect. Partitioned approaches are typically used for large-scale engineering FSI problems. This is not only because of the problems associated with the complexity of monolithic approaches, but also due to software availability. If no existing monolithic solver is available for use, which was the case for this thesis, one would need to be built from the ground up, whereas partitioned methods can take advantage of more readily available existing software.

\subsection{Partitioned FSI Coupling}

Partitioned methods reduce the complexity of the FSI problem by splitting up the systems of equations to their own respective solver. Under this approach, software modularity is a significant advantage as it allows the use of specialized solution methods that are more efficient for specific problems. Unlike monolithic methods, source code access is not necessarily required for either solver. Widespread availability of commercial software can then speed up any development time required by the engineer to implement an FSI analysis. For this project, two separate and distinct solvers were used, both of which were specifically tuned for their respective structure and fluid solution methods, so the only development required was to build the coupling interface between the two. Compared to monolithic methods, when applied to large, complex problems, separate, highly specialized solvers are often better suited to solve the individual problems due to the inherent nature of the mathematics involved [10]. A partitioned approach was chosen for the VCCW project due to the use of commercial FEA software and the rapid development time associated with partitioned methods. 


\subsubsection{Partitioned Coupling Methods}

Partitioned solution algorithms can be further classified as loosely or tightly-coupled, referring to the level of enforcement of time-accurate equilibrium at the fluid-structure interface. Tightlycoupled solution algorithms improve time-accuracy by placing additional subiterations within the fluid and structural solver between the information exchange time steps. Loosely-coupled algorithms simply exchange information at each FSI step without any specific time-accurate subiterations taking place.

Partitioned methods and loosely-coupled methods in particular, are often criticized for not being time-dependent or numerically stable. These deficiencies are typically credited to the method of information exchange rather than the fluid and structural solvers, which have been validated separately. A partitioned, loosely-coupled solution can be made more accurate by reducing the time steps to an appropriate value rather than taking a sub-iterative tightly-coupled approach. Farhat shows that a loosely-coupling method can be second-order accurate and successfully applied to aeroelastic problems in compressible flow when tied to an efficient staggered solution algorithm [11]. Tightlycoupled algorithms can be used to obtain time-accuracy, but at the cost of increased runtimes and code complexity. In this project, the loosely-coupled approach was chosen both for its simplicity and rapid development time as well as to determine its success when applied to the particular type of structure that the VCCW employed.

The aerodynamic flow solution, in the form of the vector $y$ in Eq. (2.1), denotes the pressure distribution on the aeroelastic body within the fluid domain. The initial aerodynamic flow-field solution is obtained on the non-deformed geometry. Once the flow-field is established and converged, the aeroelastic body deformation is determined by the separate structural solver. The CFD mesh is then adapted to match the shape of the new deformed body as defined by the structural code. The 
flow solution is then restarted and updated based on the new geometry. Once the updated solution is obtained, the new pressure distribution along the surface is exported to the structural solver.

$$
y^{n+1}=F\left(x^{n}\right)
$$

The structural deformation solution, given as the vector $x$ in Eq. (2.2), denotes the displacements of each node of the aeroelastic body, based on the forces on the fluid-structure interface. The surface pressures defined by the flow solution are imported as a mapped, distributed load on the structure. Once the new deformations are obtained, they are exported to the flow solver for an updated surface pressure distribution.

$$
x^{n+1}=S\left(y^{n+1}\right)
$$

This loosely-coupled algorithm is commonly referred to as a conventional serial staggered (CSS) scheme $[10,12]$ and is detailed in Table 2.1 and Figure 2.1. This approach is only firstorder time accurate, but has been found to be suitable for aeroelastic simulations [11, 10, 13, 14, 15] where $n$ serves simply as an iteration or time-step counter. Note that this work only addresses the steady-state aerodynamic and structural responses.

Table 2.1: Conventional Serial Staggered Scheme

\begin{tabular}{|l|c|}
\hline $\begin{array}{l}\text { 1. Solve flow equations } \\
\text { 2. Solve structural equations } \\
\text { 3. Increment to next step }\end{array}$ & $y^{n+1}=F\left(x^{n}\right)$ \\
\hline
\end{tabular}




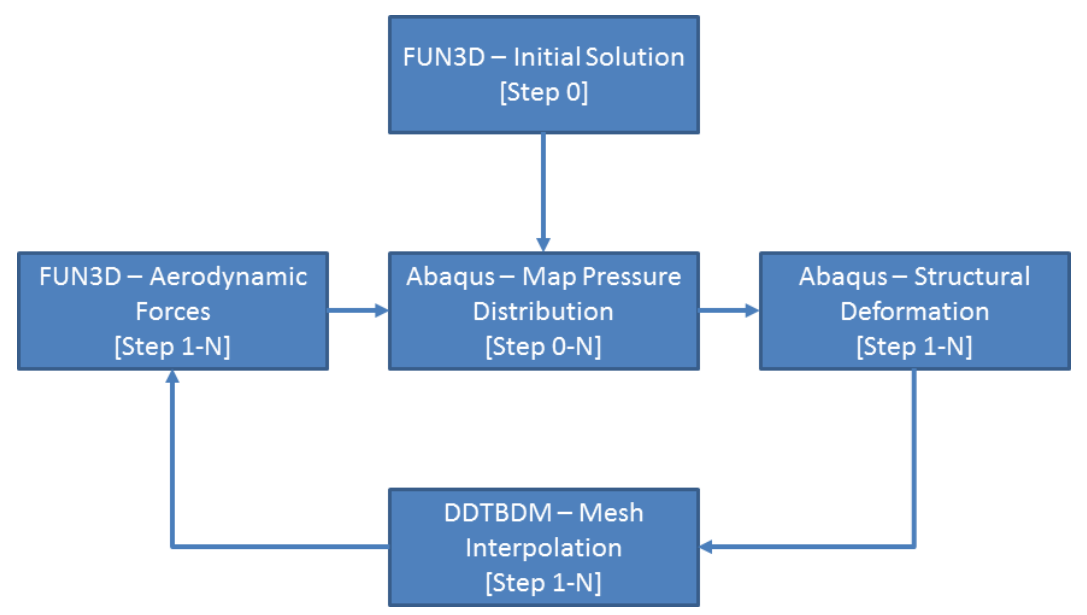

Figure 2.1: Loosely-Coupled, Partitioned Solution Algorithm 


\section{CHAPTER III}

\section{VARIABLE CAMBER COMPLIANT WING}

Two versions of the VCCW were built and tested; a $1 \mathrm{ft}(0.3 \mathrm{~m})$ bench-top model used to test the compliant mechanism, construction methods, and camber profile accuracy, and a $6 \mathrm{ft}$ model for testing under full aerodynamic loads in a wind tunnel. The $6 \mathrm{ft}$ prototype was a straight, uniform, rectangular wing with a $6 \mathrm{ft}(1.8 \mathrm{~m})$ span and $2 \mathrm{ft}(0.61 \mathrm{~m})$ chord and was the focus for this thesis. The objective of the VCCW's design was to achieve a change in maximum camber with standard NACA four digit series profiles as the target (NACA 2410 through 8410). For consistency's sake, the cambers in this report are referred to by their NACA target, rather than by the amount of actuation or actual experimental shape. A single force actuation was designed to continuously change the camber through the embedded compliant mechanism. During actuation, the leading and trailing edges traveled vertically with respect to the constant camber location at the spar. Due to its camber changes and flexible nature, the VCCW provided a unique opportunity and was an ideal candidate for a fluid-structure interaction analysis.

\subsection{Wind Tunnel Testing}

The $6 \mathrm{ft}$, full-scale prototype was fabricated with the intent of testing under full aerodynamic load in a wind tunnel. The main objective of the wind tunnel test was to demonstrate and measure the response of the VCCW under aerodynamic load through skin deflections. The Vertical Wind 
Tunnel (VWT) facility at Wright-Patterson Air Force Base was chosen because of the available flow regime and physical accessibility to the model. The VWT has a $12 \mathrm{ft}$ (3.66 m) diameter, openjet test section that offers flow speeds up to $150 \mathrm{ft} / \mathrm{s}(46 \mathrm{~m} / \mathrm{s})$. The open test section allowed for the installation of optical measurement equipment, light sources, and other equipment that was used to perform surface oil-flow visualization and digital image correlation. Testing a flexible, continuously morphing wing, and applying relatively unprecedented measuring techniques, was a considerable challenge and created a unique environment to conduct valuable research. Analyzing the VCCW required the use of multiple measurement techniques in order to get a greater understanding of the aerodynamic and structural physics.

Table 3.1: Experimental Test Configuration

\begin{tabular}{|c|c|}
\hline Freestream Velocity & $50 \mathrm{knots}(25.7 \mathrm{~m} / \mathrm{s})$ \\
Reynolds number & $1.05 \cdot 10^{6}$ \\
Mach number & 0.076 \\
Chord & $2 \mathrm{ft}(0.61 \mathrm{~m})$ \\
Span & $6 \mathrm{ft}(1.8 \mathrm{~m})$ \\
Wetted Area & $12 \mathrm{ft}^{2}\left(1.12 \mathrm{~m}^{2}\right)$ \\
Angle of Attack & $-4^{\circ}$ to $8^{\circ}$ \\
Target NACA Cambers & $2410,4410,6410,8410$ \\
\hline
\end{tabular}

Since the wing was smaller than the test section, a custom fixture had to be designed to simultaneously support the model and measure forces and moments. An in-depth discussion of the wind tunnel and the testing procedures is detailed by Marks et. al. [16], the primary engineers involved in testing the VCCW. Force and moment data, and the resulting lift and drag, was acquired by force couples embedded in the support structure. Fixture alignment issues created considerable complexity that required further analysis to be fully understood. This meant that the data was not available 
for use in the FSI analysis. Local section lift coefficients were calculated by surface static pressure taps located near the mid-span of the wing. The test matrix is summarized in Table 3.1. Note the depiction of the wind tunnel test configuration in Figure 3.1(a) and a sample of the smoke flow visualization completed during the test in Figure 3.1(b). The net shown in Figure 3.1(b) was not used during the main test runs.

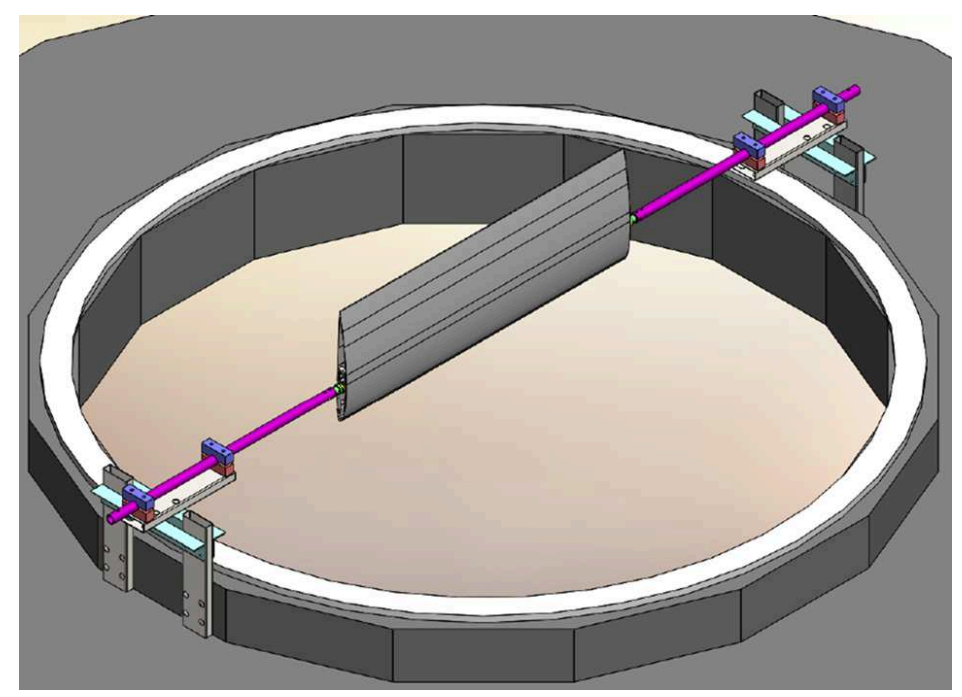

(a) Tunnel Configuration

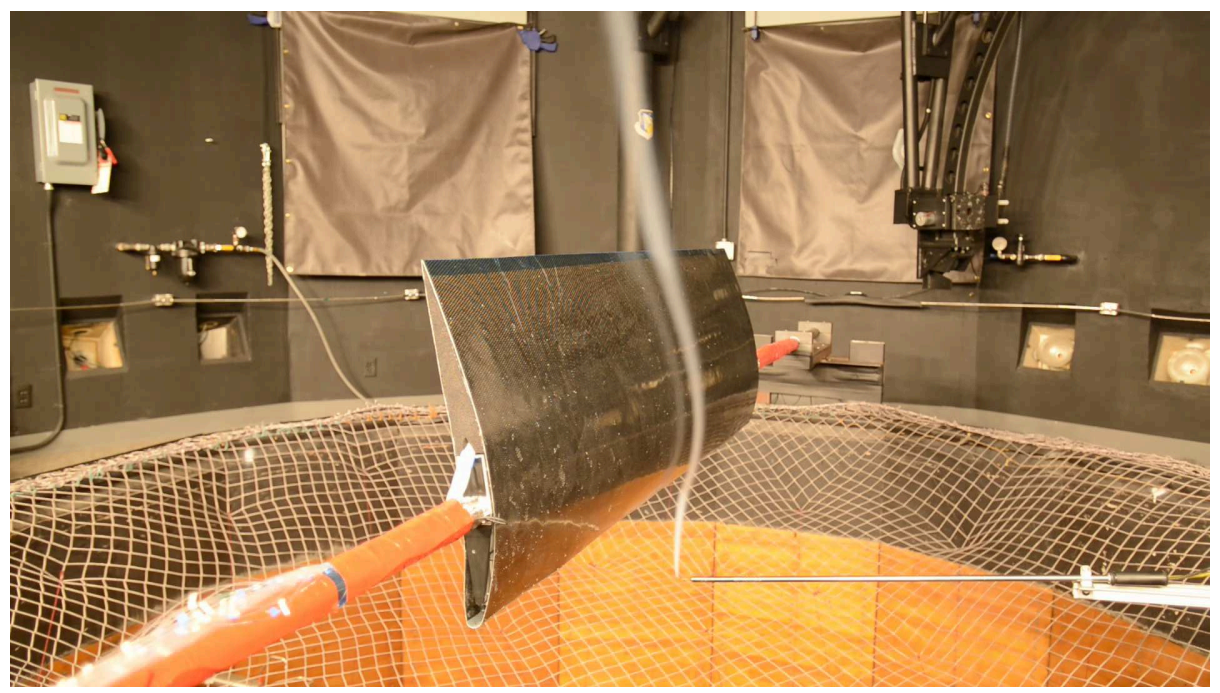

(b) Flow Visualization

Figure 3.1: $6 \mathrm{ft}$ VCCW model installed in the WPAFB Vertical Wind Tunnel 


\subsection{Digital Image Correlation}

Digital image correlation (DIC) data was taken during a portion of the wind tunnel test. The DIC system took a series of pictures of the top surface of the wing, with and without the tunnel running, which provided total displacements due to aerodynamic loading in full 3D space. Unfortunately, due to the physical restraints of the test section size, images were only taken of the top surface, leaving the leading edge and lower surface unobserved. For future testing of this type of structure, it is recommended that the leading edge be the focus of the imaging, if at all possible, due to the specific construction methods used on the VCCW and its effects on the total wing aerodynamics.

The digital image correlation setup consisted of two high resolution cameras (4008 x 2672 pixels) mounted within the test section of the tunnel. While still in the test section, they were out of the flow due to the open-jet nature of the tunnel. The cameras took two simultaneous images which were then combined for a single composite view of the top surface of the wing. The physical restrictions of the wind tunnel created a challenge when it came to the DIC setup. The optimal separation distance for this type of photography is $25^{\circ}$ but only $22^{\circ}$ was possible, so the camera placement was not ideal. Normally the out-of-plane displacement accuracy is $20 \mu \mathrm{m}$ but only $40 \mu \mathrm{m}$ was obtained in the experiments. In-plane accuracy was achieved to be within $10 \mu m$ [16].

The image outputs from the cameras were processed and aligned to create 3D surfaces which provided the closest one-to-one comparison of experimental to FSI analysis results. The raw surface DIC data can be seen in Figure 3.2 for each camber configuration at $0^{\circ}$ angle of attack. Since there was no easy datum to choose for the origin on the flexible wing, each surface was overlaid in order to find any common region between cambers. The best location turned out to be the spar which can be easily seen in Figure 3.2. The colors in the figure correspond to the different camber configurations. This location was chosen to align the experimental (DIC) and simulated (FSI) data sets. Due to the fact that the width of the DIC data did not extend to the full width of the wing, the center span was 
chosen to align the results. Alignment issues posed a problem when comparing experimental and computational results. Local twist variations along the span, and out-of-plane rotations with regard to the top surface of the wing, made it difficult to directly compare surface displacements.

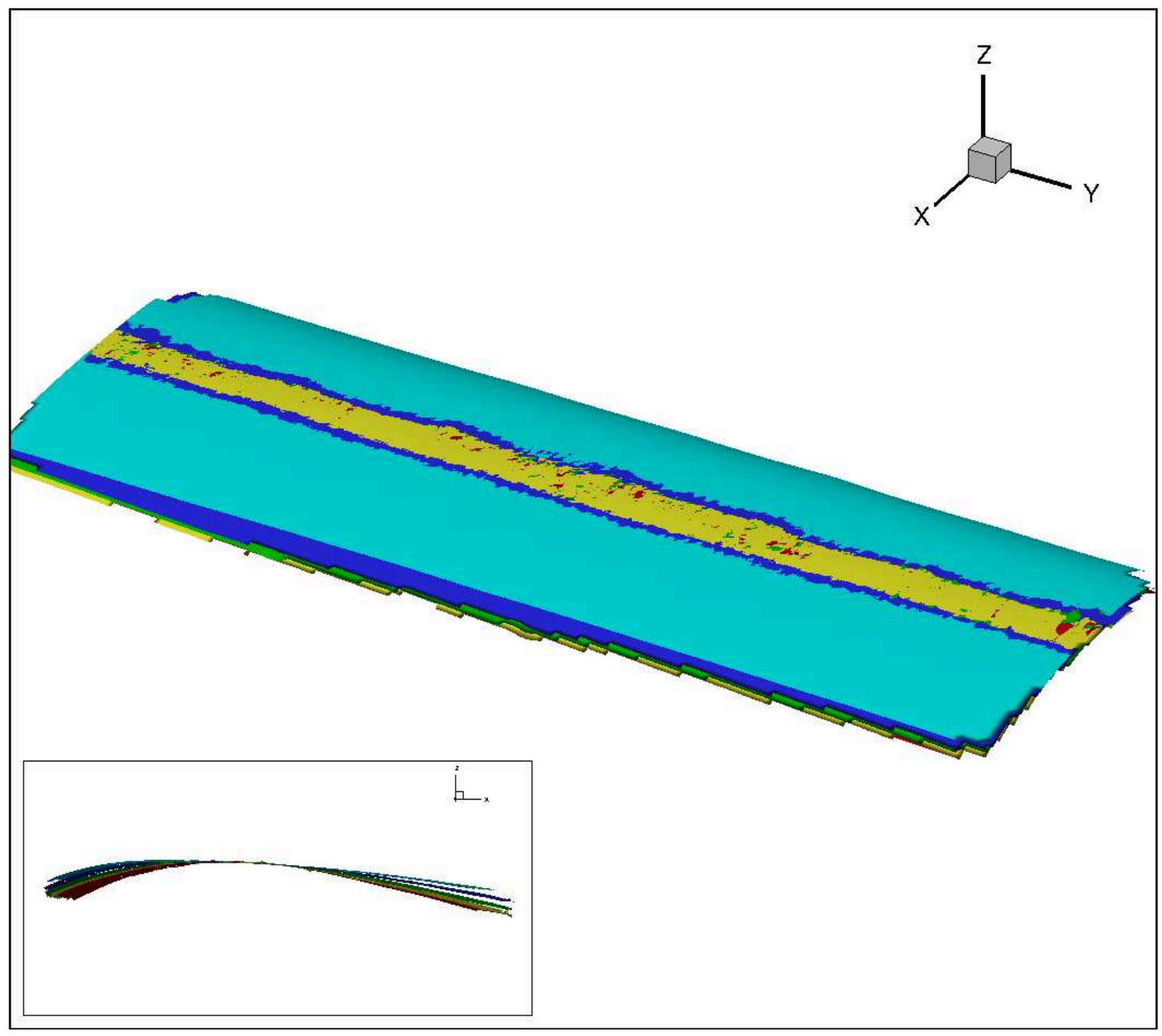

Figure 3.2: Raw DIC data surfaces for 4 cambers at $0^{\circ}$ angle of attack 


\subsection{Optical Scans}

One of the primary difficulties associated with the VCCW project was knowing the specific shape of the wing. Normally, testing on aerodynamic test articles is done with rigid, often highly precise, machined structures and materials. Typically, the exact shape is known before the test and any significant flexing is designed to be highly unlikely. With the VCCW, this was not the case, since it flexes even under its own weight. While certain NACA profiles were set as the design targets, actual shapes varied. A 3D optical measurement system was used to take scans of the prototype, albeit outside of the tunnel, to provide details about the shape of the wing, which included sectional wing profiles and a 3D mesh of the entire wing. By completing optical scans of the VCCW in its different configurations, these profiles were used to calibrate the actuation system which controlled the compliant mechanism. The intent was to know the inputs required to obtain each target camber. The 3D mesh created by the optical scanning software was also used in a CFD study, detailed in Section 5.2, to observe the effect of the differences between the wing geometry used in the wind tunnel and the idealized geometry used in the FSI analysis. Note in Figure 3.3 the high variability of the local twist and maximum camber location along the span. Figure 3.4 summarizes the impact that varying local angle of attack due to the twist has on the coefficient of lift [1]. This variability in twist and maximum camber manifested itself in the local angle of attack experienced by the wing. Since the VCCW flexes under its own weight, the repeatability of the surface shape between the laser scan and tunnel configuration is questionable. Only separate pieces of information were available at each point, so the data from the scans and DIC surfaces were then combined to estimate the actual shape

of the model. Throughout this report, the angle of attack from the experimental data is taken relative to the main spar, while the FSI results are based on the orientation of the chord line. 


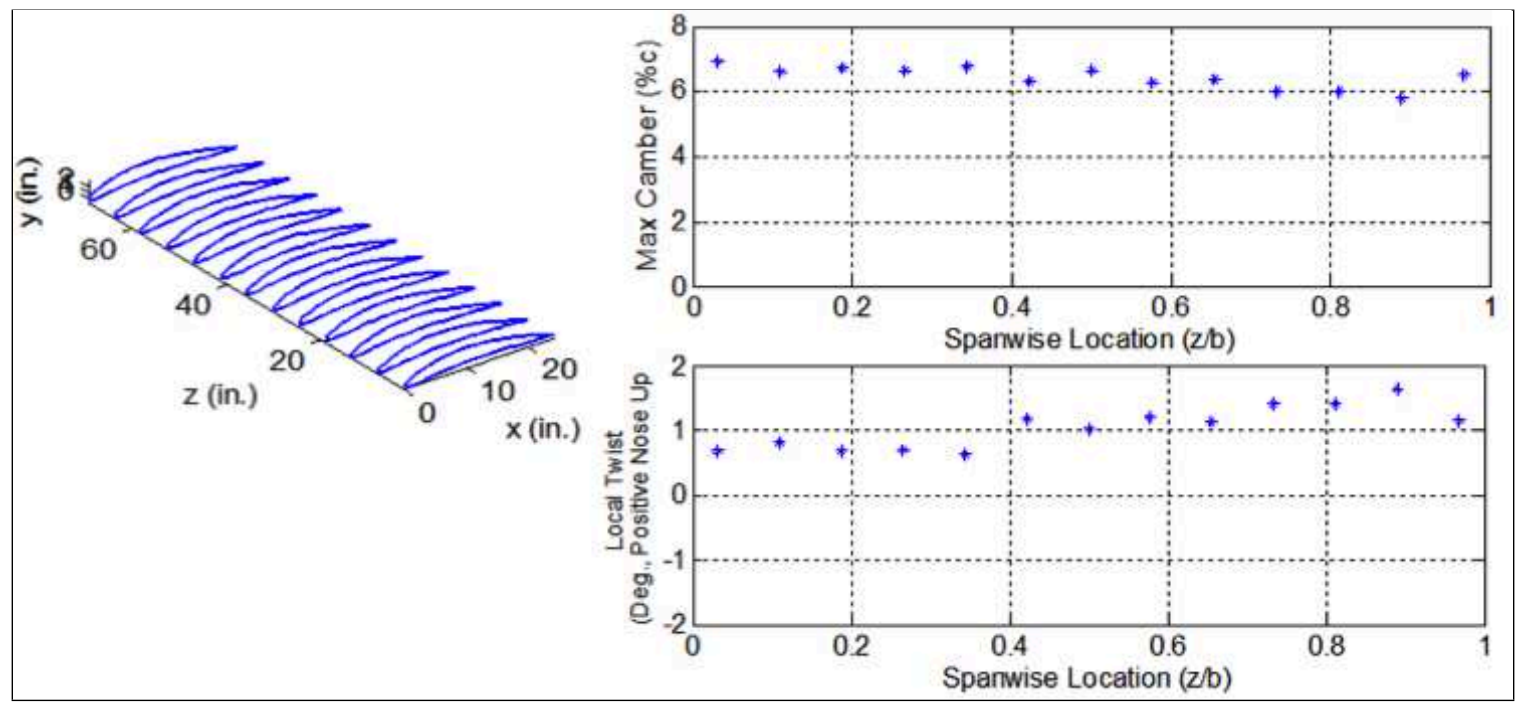

Figure 3.3: Sample measured profiles specifying maximum camber and local twist [1]

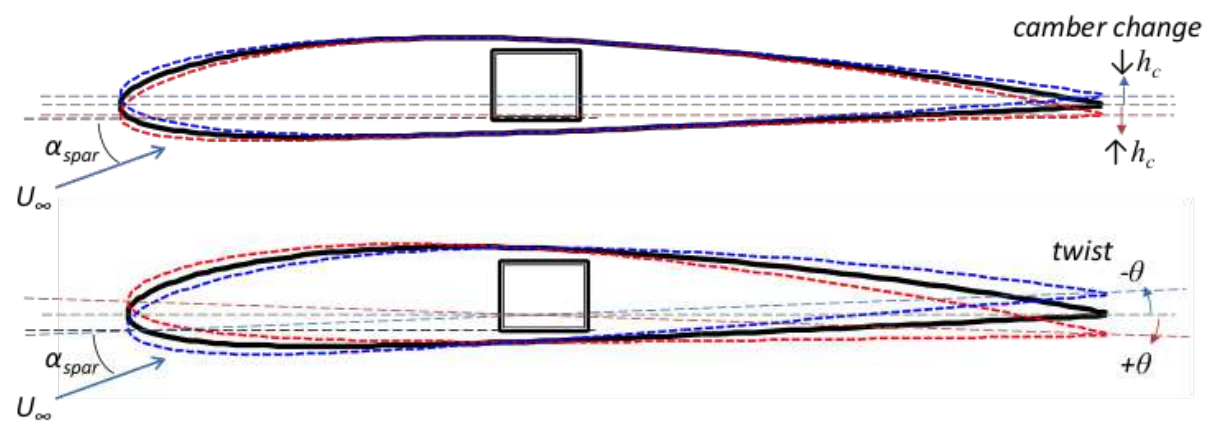

(a) Camber variability

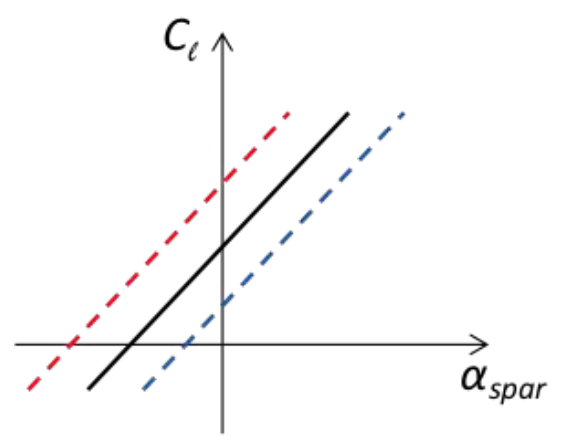

(b) $\mathrm{Cl}$ variability

Figure 3.4: Local twist effects on camber and coefficient of lift [1] 


\subsection{Oil Flow Visualization}

During a portion of the wind tunnel testing, the upper surface of the wing was coated with a mixture of oil and dye to visualize surface oil flow. The shear stress created by the airflow over the wing produced a time-independent oil flow pattern. Figure 3.5 is a sample of the results obtained by the wind tunnel staff for the NACA 2410 and 8410 target camber configurations at an angle of $8^{\circ}$. While $8^{\circ}$ was not part of the FSI analysis segment due to the higher amount of transient aerodynamics, the figure shows similar results to what will be presented in a later section focusing on the differences in CFD results between the ideal FSI configuration and the scanned wing that was tested. The oil flow showed a qualitative representation of the considerable span-wise flow variation, specifically relating to transition, separation, and tip effects. The behavior shown here was similarly reproduced in Section 5.2 where CFD was performed on the geometry provided by the optical scans.

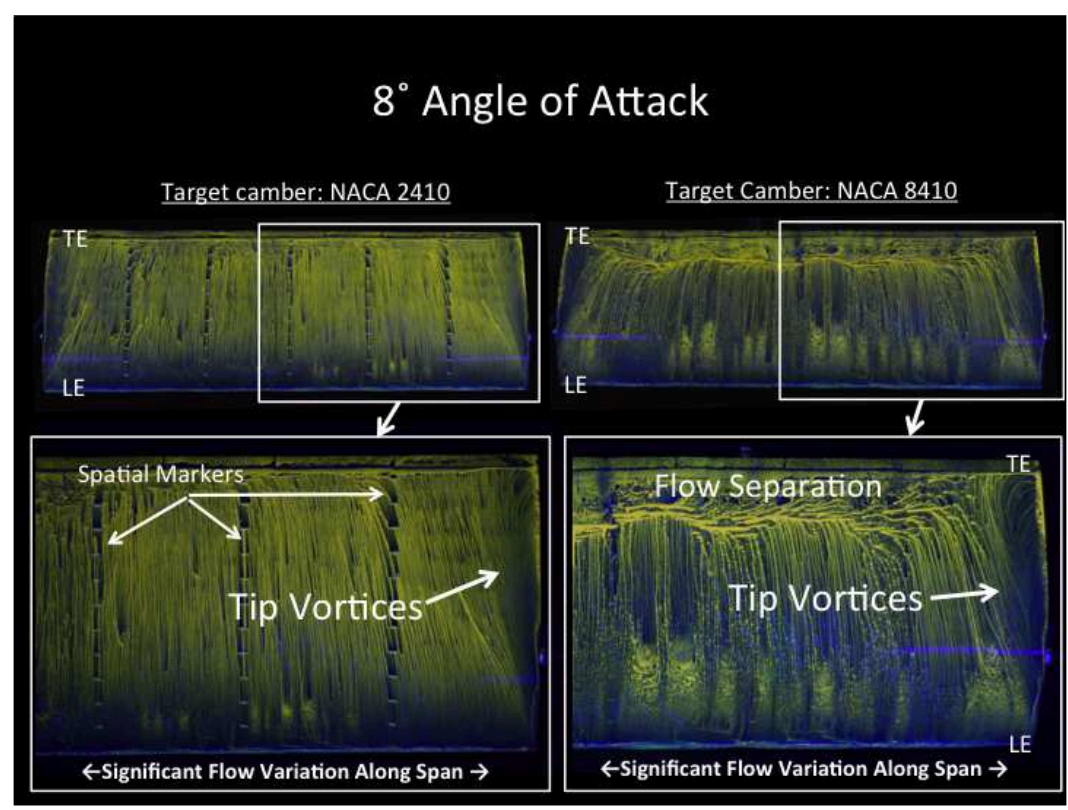

Figure 3.5: Oil Flow for NACA 2410 and 8410 at $8^{\circ}[1]$ 


\section{CHAPTER IV}

\section{FINITE ELEMENT ANALYSIS}

The FEA model used for this project was built during the stress analysis and design phase of the VCCW project and was then provided for use in the FSI analysis. Only minor modifications were needed in order to facilitate the communication of data between solvers. These included running Python scripts to extract the outer-mold-line of the wing, which was the only pertinent information needed by the CFD solver, and importing the pressure loads from the CFD solution once it had sufficiently converged. The mesh interpolation code did not accept the native Abaqus formatting, so a Python script was used to write the ASCII versions in NASTRAN format which described the model and nodal displacements, which the mesh interpolator (DDTBDM) could support. This script scanned the model for the nodes, cells, and connectivity information on the outer mold line and the respective displacements and rotations at each node. Because the external aeroelastic module was used in FUN3D, the wing boundary surface, with the respective pressures at each node, was exported at the end of each FSI iteration. These files were then parsed with Python and placed in a format suitable for Abaqus to create a mapped pressure surface on the outer-mold-line of the wing. Abaqus handled the interpolation and mapping of the pressure loads automatically.

The physical prototype was constructed of aluminum, composites, and 3D additive materials. The specific design of the model is covered in more detail by Joo [1] and Marks [16]; its complex 
internal structure is beyond the scope of this thesis. The FEA models for the $1 \mathrm{ft}$ and $6 \mathrm{ft}$ versions were used to test the methods of actuation and to simulate the final camber profiles during the design phase. Each model used a uniform 2D pressure distribution, calculated in XFOIL, to simulate the maximum aerodynamic loading and corresponding stress and strain. While adequate to simulate maximum loading, the $2 \mathrm{D}$ pressure distribution did not represent the tested conditions, and neglected 3D effects like wing tip vortices, which reduce the overall lift generated by the wing. Computationally, both versions of the model were constrained by fixing each spar end while the remainder of the model was allowed to flex, matching the physical constraints. Multiple versions were created of the $6 \mathrm{ft}$ model which included the wing both with and without the support structure. Most of the analysis presented in this thesis uses the results from the $6 \mathrm{ft}$ FEA model without support structure. Future work could increase the fidelity of the analysis by including the support structure, which would show additional bending along the span. Gravity could also be included to account for the model bending under its own weight due to the vertical arrangement of the tunnel.

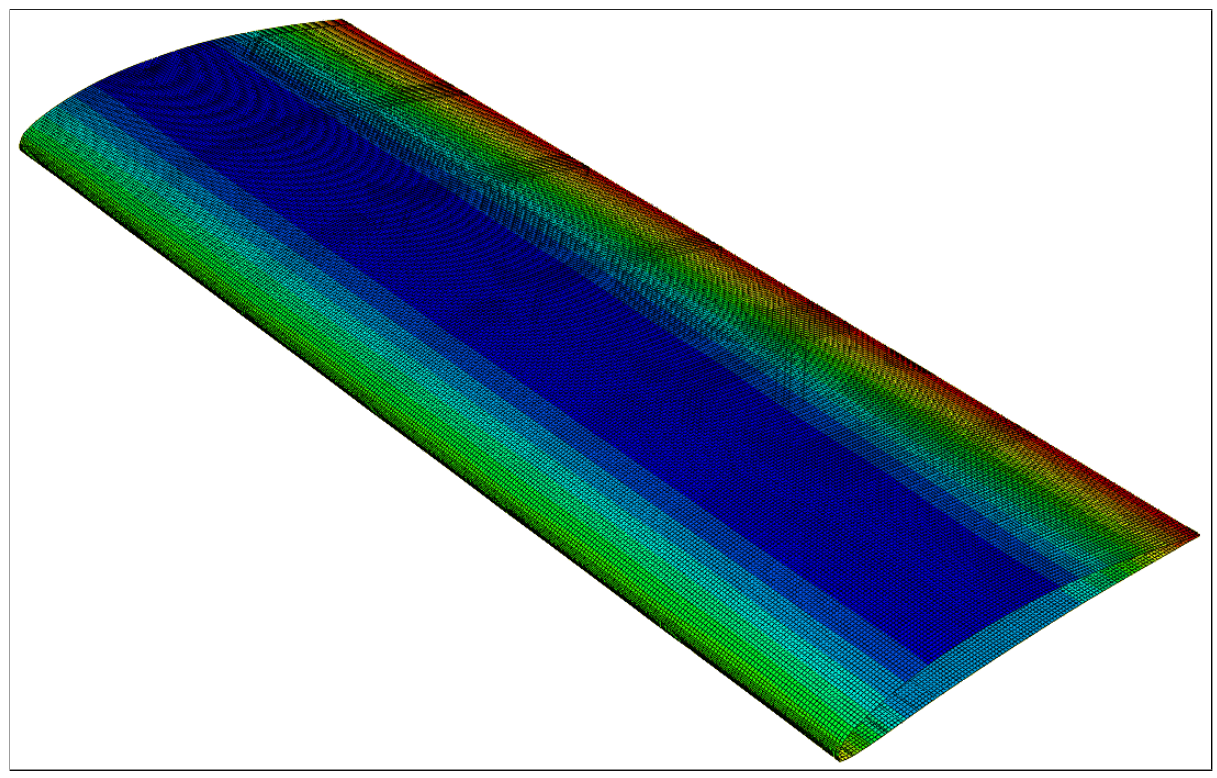

Figure 4.1: Abaqus FEA mesh configuration showing displacements colored by magnitude 


\subsection{Material Modeling}

Neither of the FEA models included the leading edge material of the wing because the physical prototype consisted of a highly nonlinear compliant composite material that was difficult to model. The flexible composite material, used to allow the high range of motion during camber changes, proved to be difficult to model within Abaqus and was the limiting factor in the FSI analysis presented. Part of the difficulty in coupling CFD and FEA together, was that the CFD surface model needed to be watertight, but the FEA model did not. The mesh interpolation code was able to handle small gaps or disparities in geometry, but could not account for the entire leading edge. The leading edge also contained the highest amount of curvature and non-linear deformation due to the material type; the difficulties this presented in modeling had considerable impact on the deformations and strongly influenced the results of the entire project.

Numerous attempts were made at modeling a material that offered very slight resistance to movement, akin to a thin sheet of rubber. Experience with handling the physical model pointed towards similar behavior at the leading edge; however there was only limited success with these estimated models and the results never proved to be reliable. Multiple material types were tried, but all had difficulty converging to a solution in Abaqus, and when they did, the leading edge shape buckled considerably, as shown in Figure 4.2. The optical scans of the prototype did show that there was considerable non-uniformity and some buckling in the leading edge, but not in the same manner as the FEA models. Material test data was taken on a specimen of the skin, but the results were highly variable, as shown in Figure 4.3, and only served to get a baseline estimate for the material model within Abaqus. Numerous test data fitting techniques were tried but none proved successful for all camber configurations. The runs which included leading edge material types took considerably longer to converge to an answer within Abaqus, and oftentimes did not converge at all for the high camber configurations. The simulations without leading edge material included ran 
successfully and could simulate each of the profiles. Future projects that attempt to simulate similar testing and structures will need to focus heavily on material properties since it has such a significant impact on the final result.

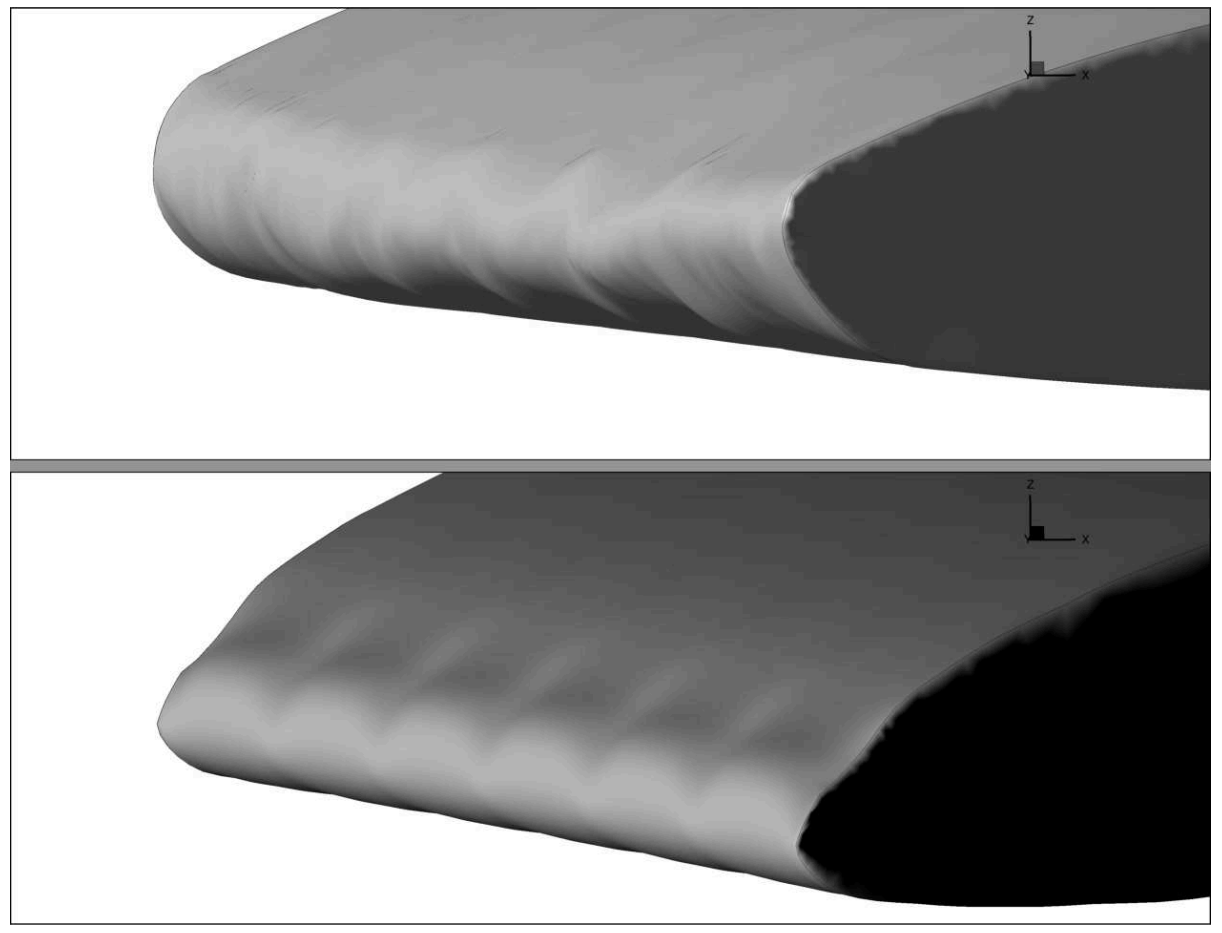

Figure 4.2: Leading Edge Material Estimate - Behavior Comparison between computations (top) and experiments (bottom) 


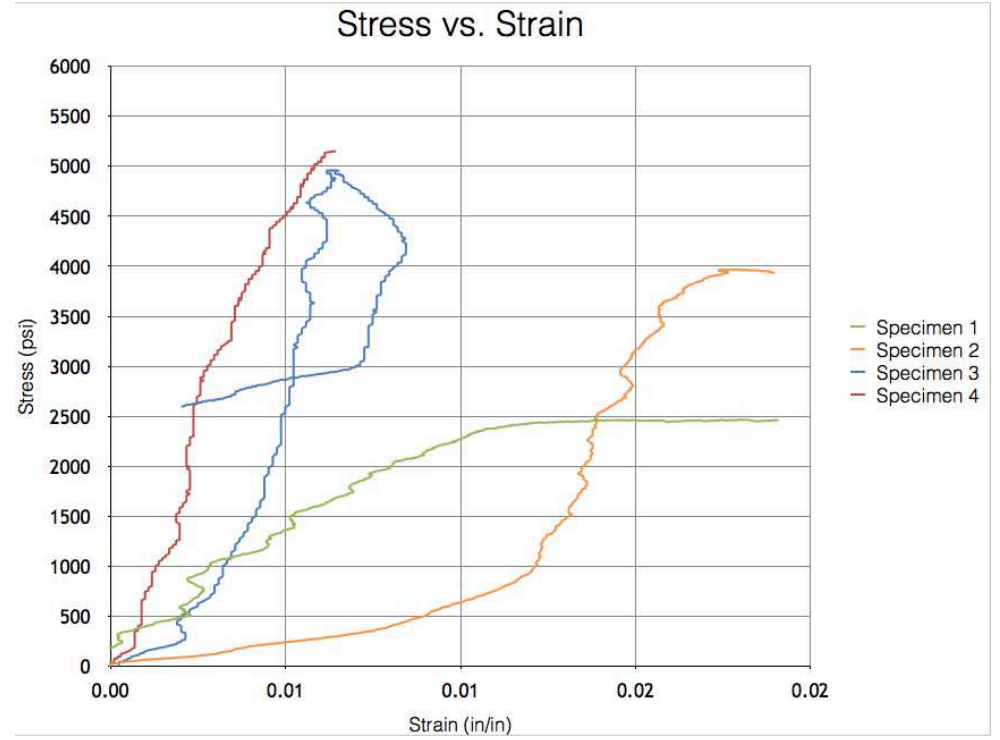

Figure 4.3: Material Test Data Specimens

\subsection{Compliant Mechanism Actuation}

The FEA model contained the internal structure of the VCCW, which was comprised of a compliant mechanism, ribs, spar, and skin. The model was then actuated, similar to the physical prototype, to the desired camber configuration. Profile shapes from the optical scans were used as references to match the level of actuation in the FEA model to profiles tested in the wind tunnel. Within Abaqus this process was achieved in two steps: The first step applied the actuation force, and the second step enabled the aerodynamic load in the form of a pressure field on the surface of the model. The actuation force of the compliant mechanism was constrained and held constant so that there was enough force to hold the camber position throughout the simulation. The actuation of the compliant mechanism in the prototype had similar behavior, but there was an amount of slippage in the actuation of the leading edge. This ultimately allowed movement of the leading edge, even after the actuation was completed, which created an uncertainty within the experiment concerning the profile shape of the wing. There was no straightforward way of modeling this in the FEA software, 
so the decision was made to simply constrain the actuation. This then created a source of error due to the fact that any significant amount of aerodynamic pressure on the wing could induce movement and create discrepancies in the data.

\subsection{NACA Profiles - FSI versus Experiment}

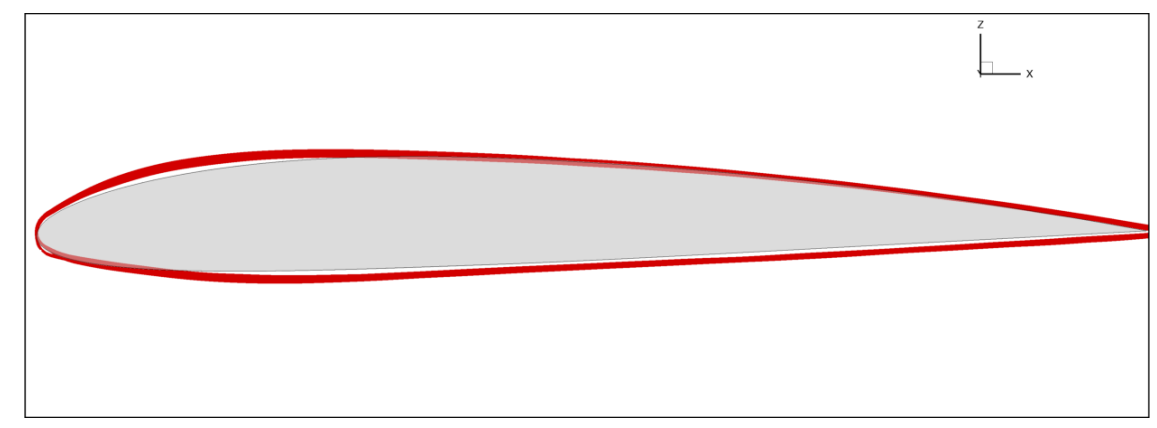

Figure 4.4: NACA 2410 Profile Comparisons

While the FEA-actuated profiles were similar, there were a few key differences. Figures 4.4 and 4.5 show the different profiles comparing the FEA results to the scans. The results of FEA actuation are shown in gray and the profiles taken from the scans are shown in red. For both cambers, the experimental wing is thicker in the first half of the chord. The leading edge of the FEA-actuated NACA 4410 has topology issues due to some of the mesh interpolation errors discussed later. It was not possible to load the scanned geometry into Abaqus to use as a starting position, so an ideal NACA 2410 was used. This is another one of the sources of error seen in the FSI analysis, since both the FEA and CFD simulations had to assume ideal conditions, whereas the experimental data did not directly match ideal NACA profiles. 


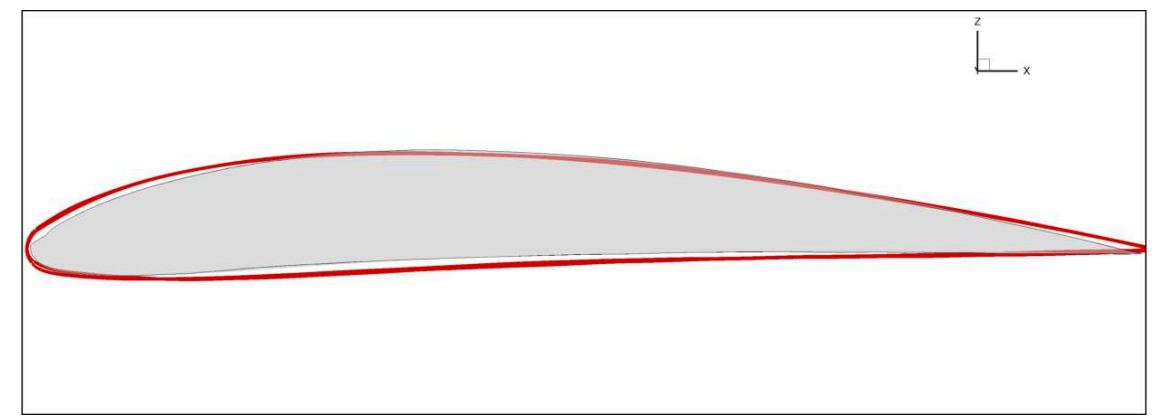

Figure 4.5: NACA 4410 Profile Comparisons

\subsection{FEA Runtime Statistics}

Run-times for the FEA models were significantly less than the CFD simulations, as expected. The FEA model had 112,000 nodes and 110,000 quadrilaterals and tetrahedral elements. The initial FEA solution took roughly 20-30 minutes on six Intel Xeon cores and restarted solutions took about 5-10 minutes each, depending on the angle of attack and camber. Higher camber cases took longer to run due to the increased level of deformation within the FEA model. Material models also significantly impacted the runtimes. 


\section{CHAPTER V}

\section{COMPUTATIONAL FLUID DYNAMICS}

The primary CFD model used in the FSI analysis was a simple, rectangular, $6 \mathrm{ft}$ wing in freespace with no tunnel or support structure interference, and the flow was assumed to be steady-state. Any time-dependent effects were averaged out due to the nature of RANS CFD. The baseline mesh used was a 3D unstructured tetrahedral mesh that contained 2.77 million nodes. A view of the wing boundary surface mesh as well as a slice down the center of the volume is shown in Figure 5.1. A mesh resolution study was performed on the lift and drag coefficients using the flow conditions of the wind tunnel test, with both medium and fine meshes containing 11.9 and 49 million nodes respectively. The cases were all run second-order accurate in space with the Spalart-Allmaras [17] turbulence model and an average y-plus value of less than one. CFD run-times for the baseline mesh were 1 hour on 240 Intel Xeon X560 cores for the initial solution and approximately 30 minutes on 120 Intel Xeon X560 cores for each restart. The initial solutions on the medium and fine mesh took 4.5 and 20 hours, respectively, using the same hardware configuration. The grid convergence study was conducted at angles of attack of $-4,0$, and 6 degrees, at an airspeed of 50 knots and a Reynolds number of $1.05 \cdot 10^{6}$. The results from this study can be seen in Figures 5.2(a), 5.2(b), and Appendix A. As can be inferred from these figures the relatively coarse baseline mesh is gridconverged and due to its much lower computational cost will be employed for the remainder of this thesis. For the FSI analysis, the same NACA 2410 initial mesh and solution was used for every case 
at each respective angle of attack. Any deformations and camber changes were due to the solution obtained by the FEA solver.

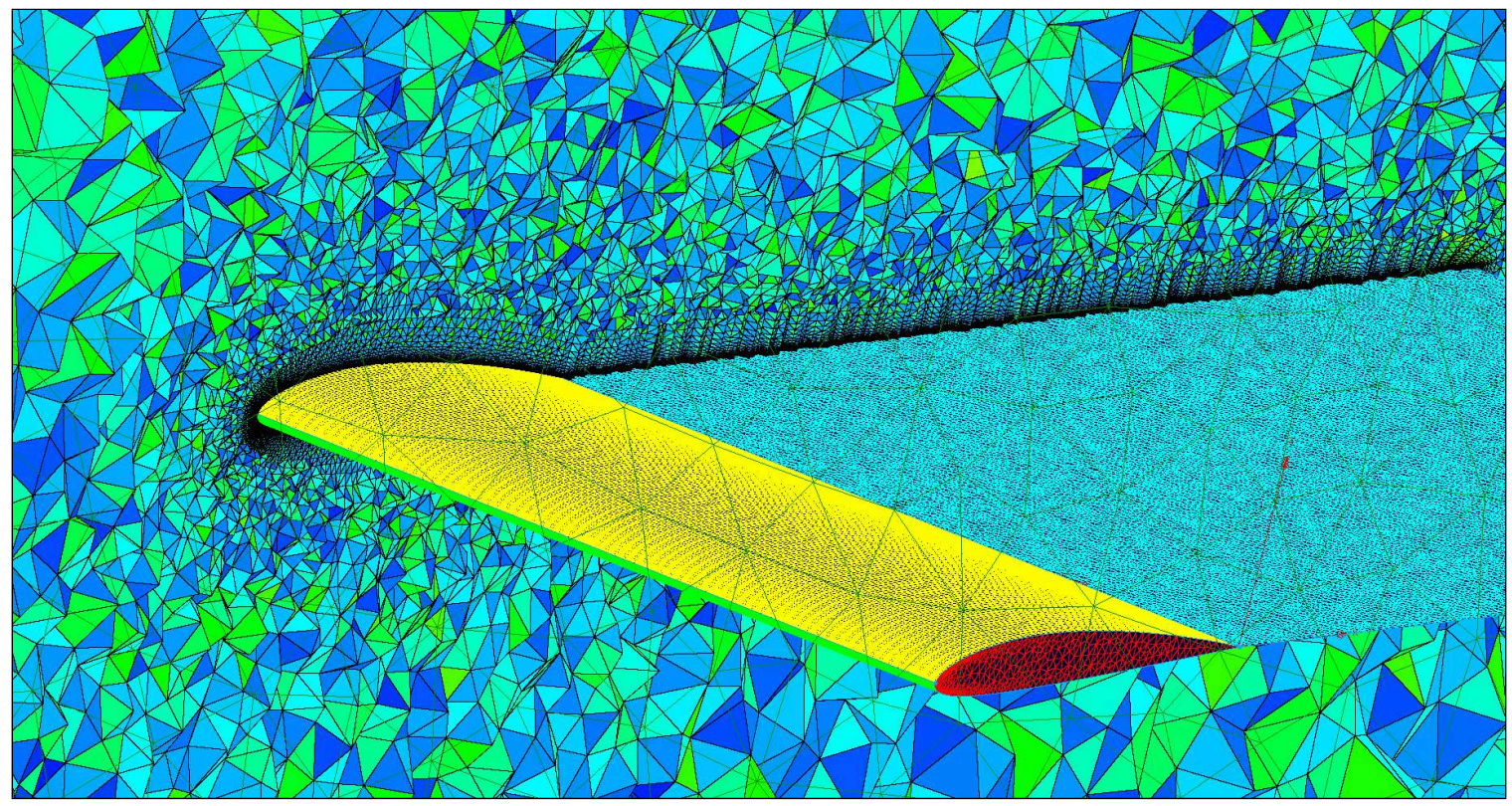

Figure 5.1: CFD mesh configuration (slice at 50 percent span)

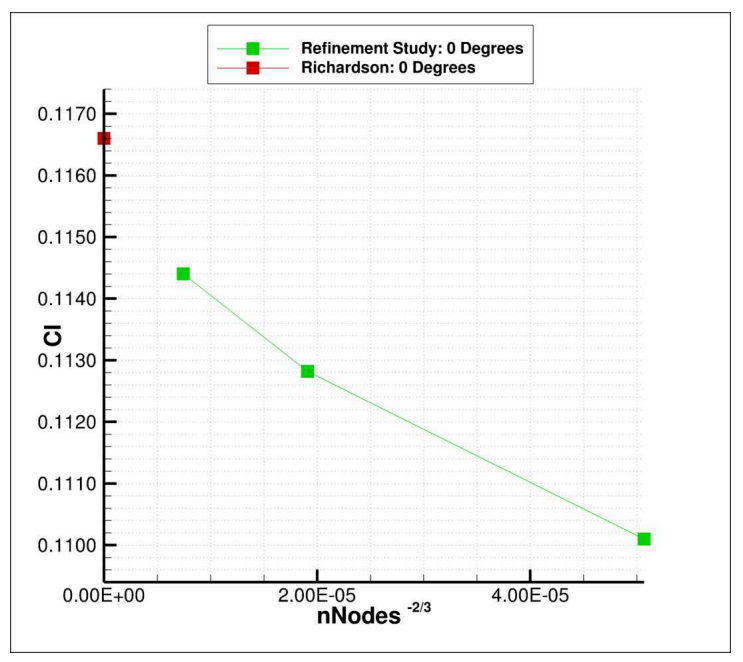

(a) Coeff. of Lift: 0 degrees

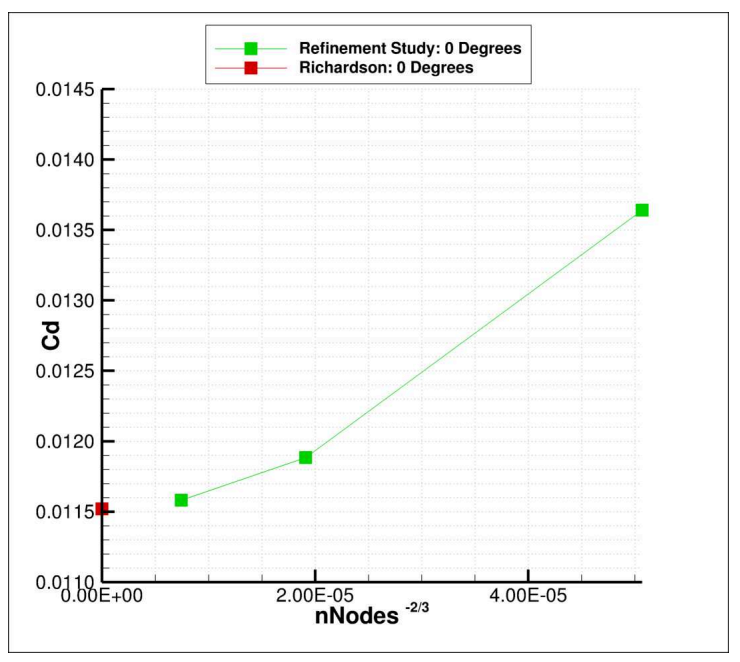

(b) Coeff. of Drag: 0 degrees

Figure 5.2: CFD Mesh Resolution Study: Initial FSI configuration (NACA 2410) 
Neither the FEA model nor the physical wing had caps at the end, leaving it open to air flow, but the CFD solver requires a watertight mesh. The wind tunnel model had foam, shown in Figure 3.1(b), inserted into the end sections to close off the majority of the sides. The sharp edges and topology of the wing caps changed considerably between a NACA 2410 and 8410, which made the caps difficult to properly mesh. This ultimately drove the decision to use an unstructured tetrahedrally dominated mesh. FUN3D also contains additional mesh deformation utilities specifically designed to untangle tetrahedrals during the deformation stage. Creating the right mesh to work for this problem became more of an art than science. Once a mesh was found to deform well in all cases, the configuration was frozen and used throughout the test matrix.

\subsection{FUN3D - External Aeroelastic Mode}

In order to get the partitioned FSI method to work, Abaqus and FUN3D had to be able to transfer pertinent data to each other. To enable this in FUN3D, the external aeroelastic routine was utilized which exports specified boundary surfaces at desired iteration increments. These exported boundary surfaces contained the force data needed to interpolate to a separate structural solver. Once the new shape was found by the FEA model, the newly deformed surface shape was inserted in place of the prior boundary surface as long as the same mesh structure was observed (same number of nodes and cells). FUN3D then deformed the volume mesh around the new surface mesh. Each FSI simulation started with the rigid model in a steady CFD simulation with the established flow-field. Later steps restarted the solution and inserted the new surfaces that were deformed by the FEA solver. Figure 5.3 shows the differences in the convergence history between cases with smaller (NACA 2410) and large (NACA 4410) camber changes. After the first two or three FSI iterations the lift and drag coefficients stop changing significantly in all cases. 


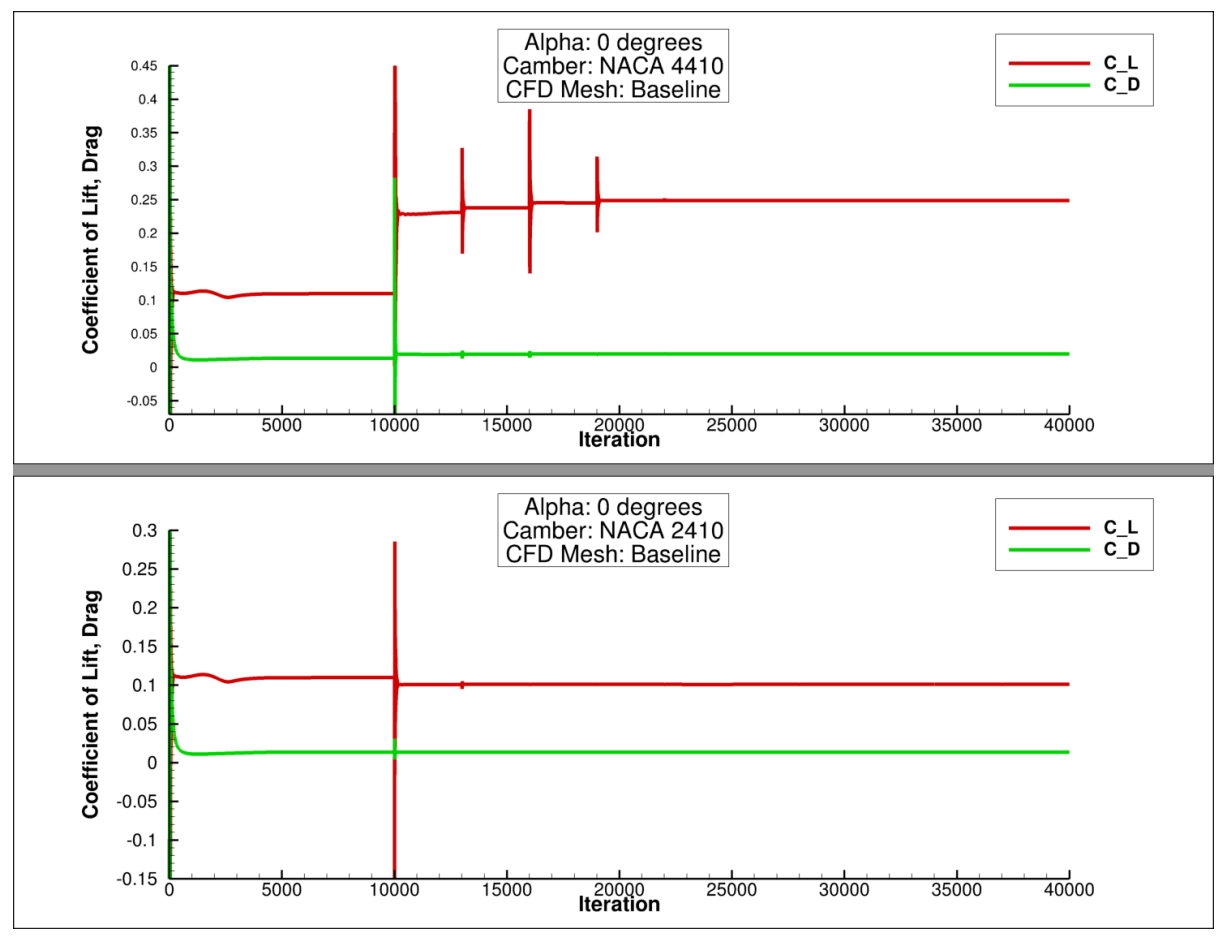

(a) Lift/Drag History

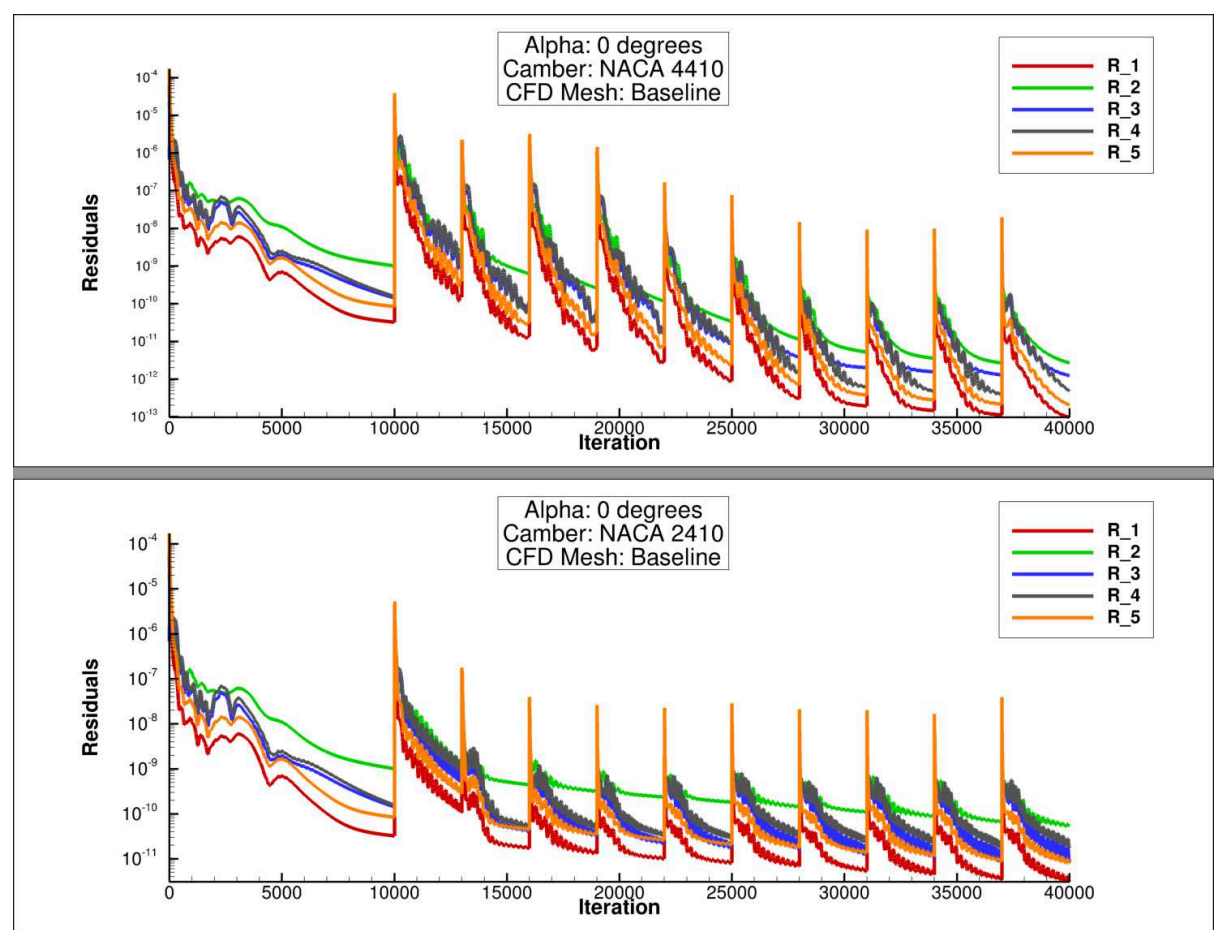

(b) CFD Residual History

Figure 5.3: CFD convergence history for the NACA 4410 (top) and 2410 (bottom) cambers at 0 degrees 


\subsubsection{Support Structure Interference Effects}

While only the wing itself was used in the FSI analysis, a small case study was accomplished to investigate the effects of the support structure. Steady-state rigid CFD simulations were completed on the wing in free-space with the supporting structure, which was modeled as a simple cylinder. These results were compared to the freestanding wing used in the FSI cases. The supporting cylinder was a source of high frequency vortex shedding that was effectively smeared out due to the steady-state CFD simulation. The intent was to observe the effects that the structure had on the surface pressure distribution of the wing. Runs were completed for the $-6^{\circ}$ through $6^{\circ}$ cases in increments of $2^{\circ}$. The lift and drag results, shown in Figure 5.4, gave a very similar lift over the entire sweep, with only slight differences. The high amount of vortex shedding present was responsible for the small deviations in the lift. If a time-dependent simulation was run for the sweep, the lift would likely experience an oscillation about the mean value given by the steady-state solutions. As expected, the drag was higher for the cases which included the support structure.

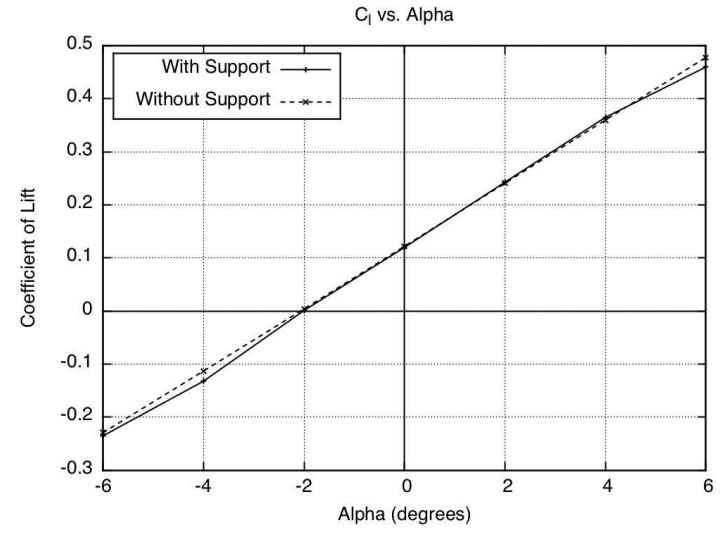

(a) Lift vs Alpha

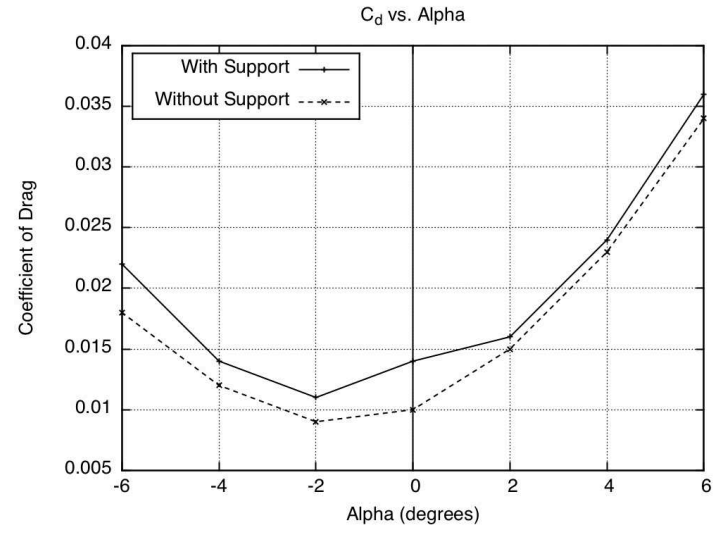

(b) Drag vs Alpha

Figure 5.4: Lift and drag comparison for support structure interference 
The differences in pressure distribution on the surface of the wing is shown as a snapshot in Figure 5.5. For an angle of attack of $6^{\circ}$, the only differences between the two existed within the first $10 \%$ of the span along the edges, as expected. As it related to the FSI simulation, this resulted in a drop in pressure force at the tips and less deflection. The remainder of the results are located in Appendix A. 


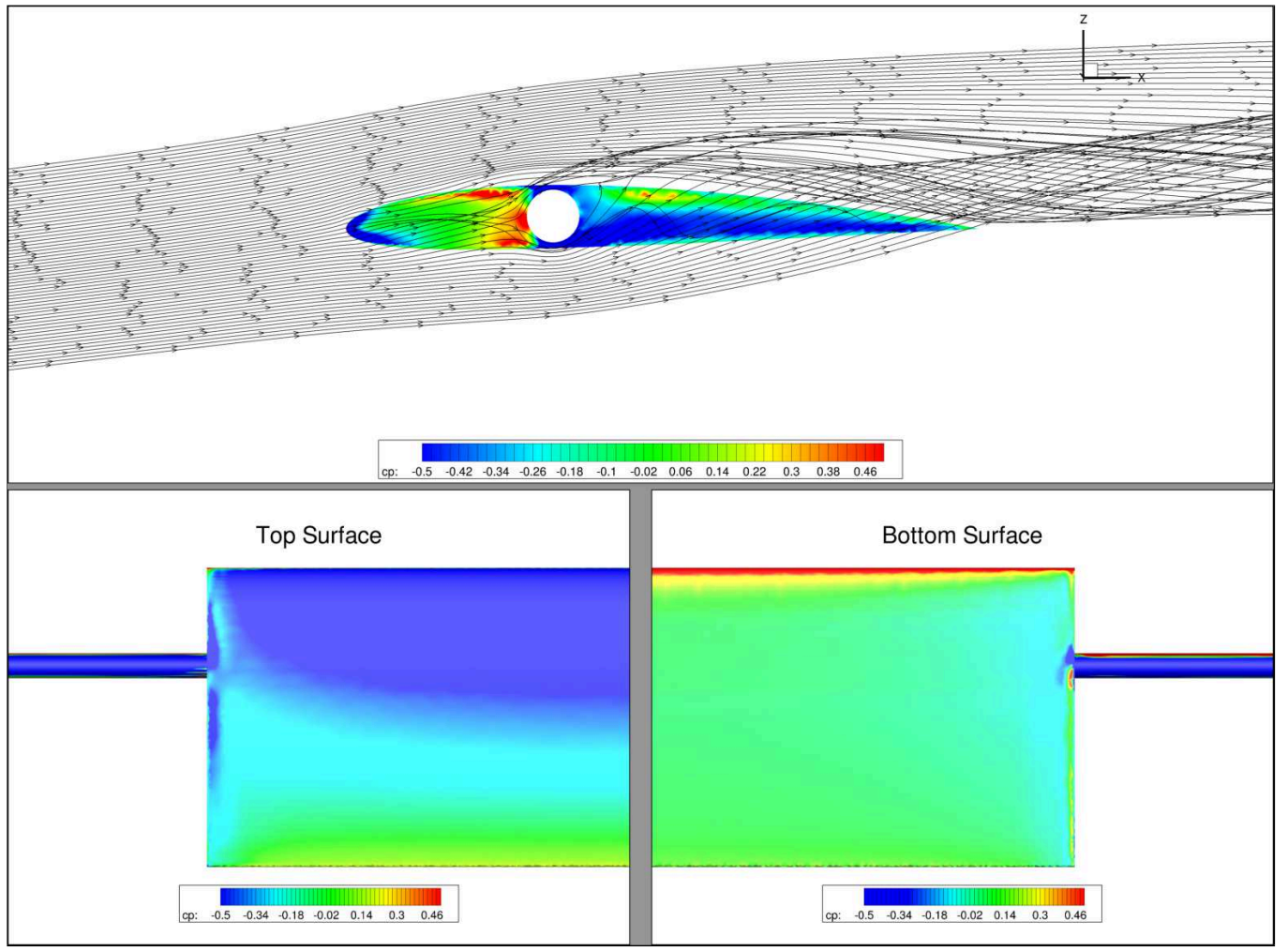

(a) With Support Structure - Alpha: $6^{\circ}$

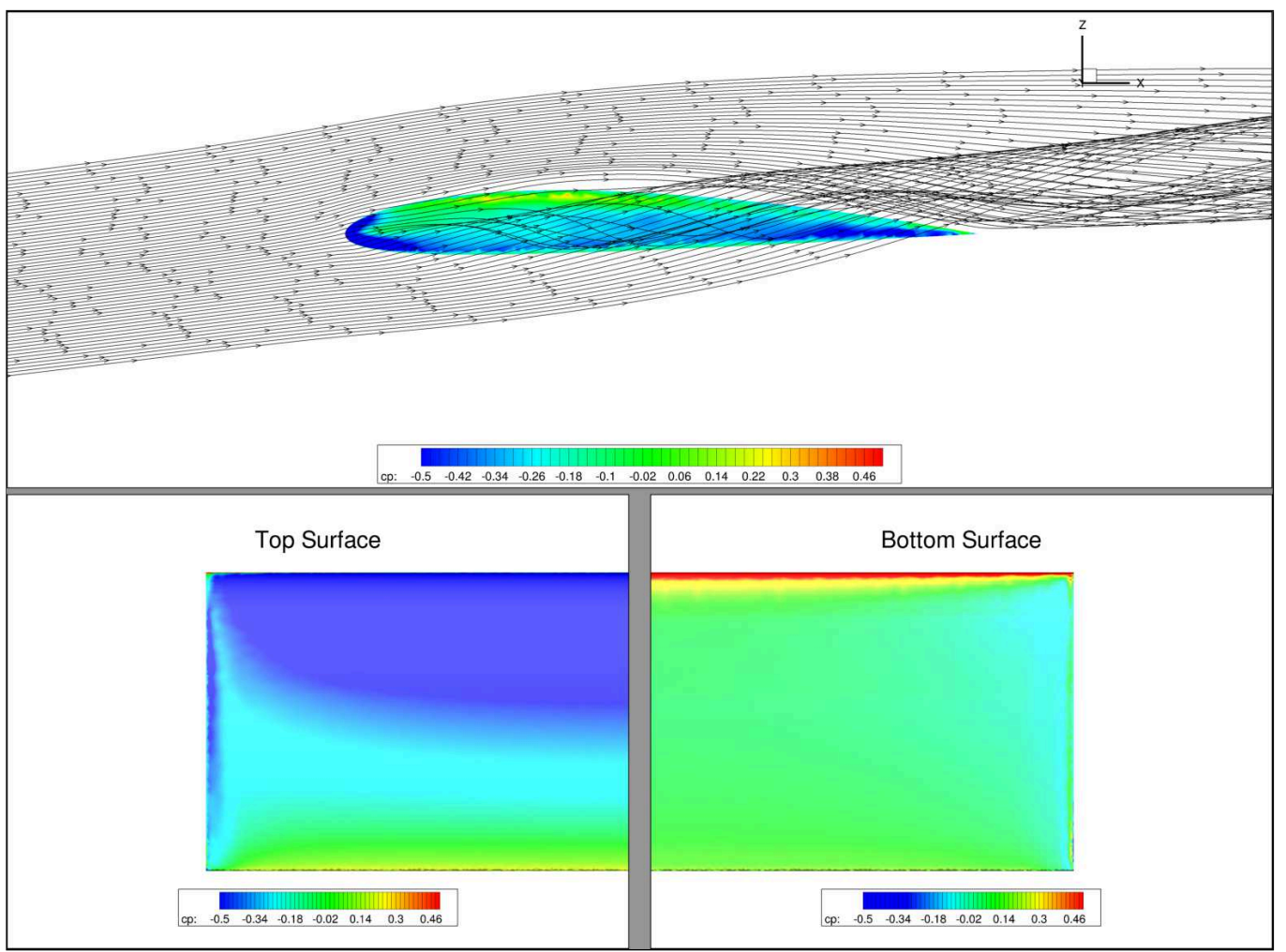

(b) No Support Structure - Alpha: $6^{\circ}$

Figure 5.5: Support Structure Interference 


\subsection{Scanned versus Ideal Geometry}

Since optical scans were taken, and at least one was converted into a useable geometry, a CFD analysis was completed to attempt to quantify the error associated with the differences in geometry between the FSI and experimental results. Each FSI analysis started with an ideal NACA 2410 profile in both the FEA and CFD solutions even though the experimental shape was considerably different than the ideal profile.

One of the optical scans, the configuration targeting a NACA 3410, was converted into a triangulated mesh and imported into Pointwise, a commercially available mesh generation software suite, to create a wing. The geometry produced by the scan was at such a high resolution that even the scotch tape that covered the pressure tap ports on the surface of the prototype was visible in the mesh, so the resolution was reduced in a mesh processing program. There were a number of issues with the mesh. Most importantly, the optical scanning equipment was unable to resolve the sharp trailing edge of the prototype, and as such, there were numerous holes throughout. These holes were patched and a blunt trailing edge was created to follow the scan as closely as possible while still providing a useable CFD mesh. An ideal NACA 3410 was also created to match the chord and span of the scan as shown in Figure 5.6 which illustrates the profiles in comparison; red indicating the ideal mesh and black depicting the scan. The considerable differences in the leading and trailing edge shapes can be seen in Figure 5.7. Both the ideal NACA 3410 and scanned wing were created as structured surfaces meshes that had the same number of nodes and cells. They were created in a way that ensured that the volume meshes generated around the two wings were as identical as possible, in order to reduce any mesh-related solution differences. The structured surface meshes were then converted to triangulated unstructured meshes in preparation for the unstructured volume mesh. During the volume mesh generation, the boundary layer growth had to be tailored to capture the surface imperfections of the scanned mesh. Some of the divots on the scan were small enough 
that they interfered with the first few layers of the mesh. The growth rates of the boundary layer were also matched for the scanned and ideal meshes. The forward-most point on the leading edge was chosen as the origin for both wings, and the angle of attack for each case was defined using the origin and chord line.

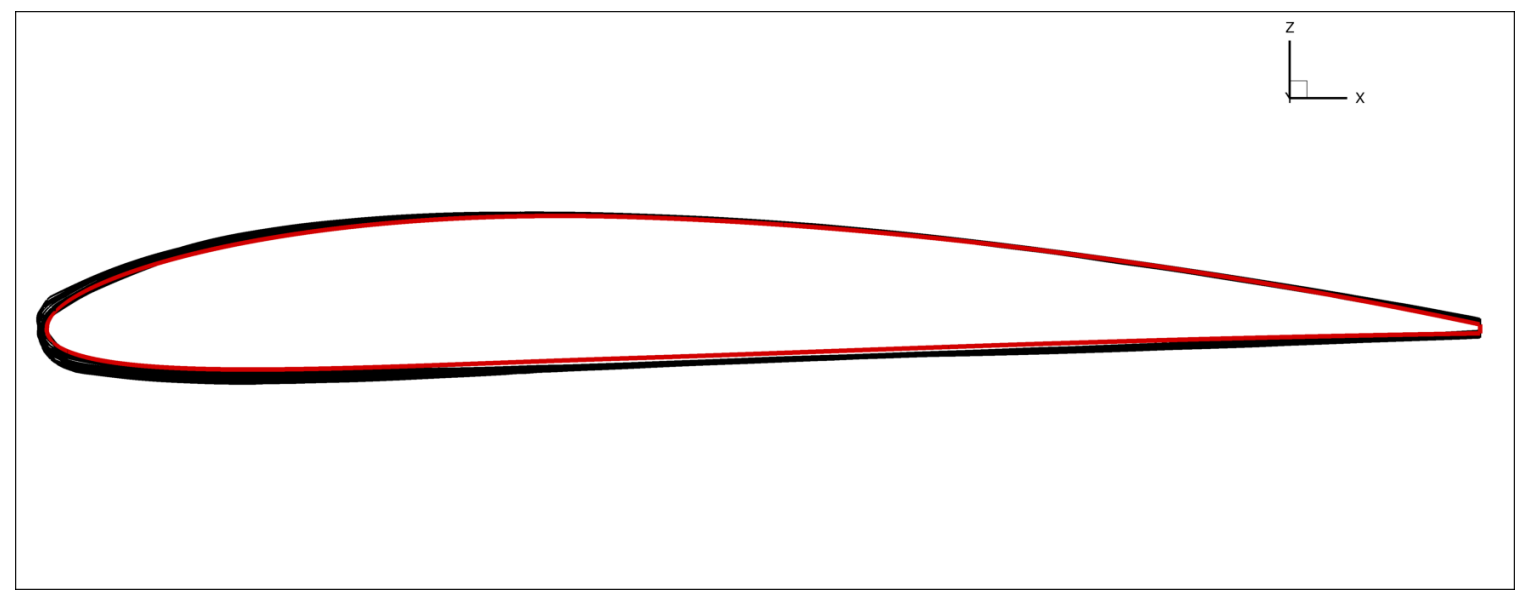

Figure 5.6: NACA 3410 Profile Comparison

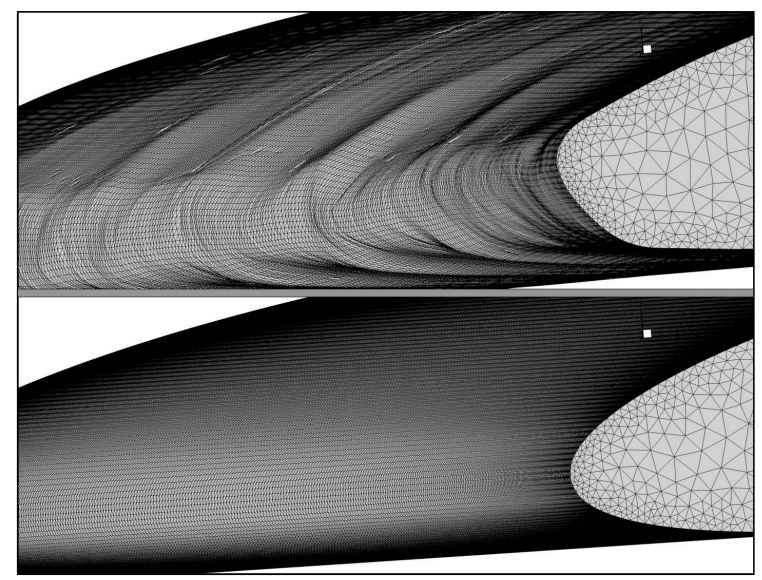

(a) Leading Edge

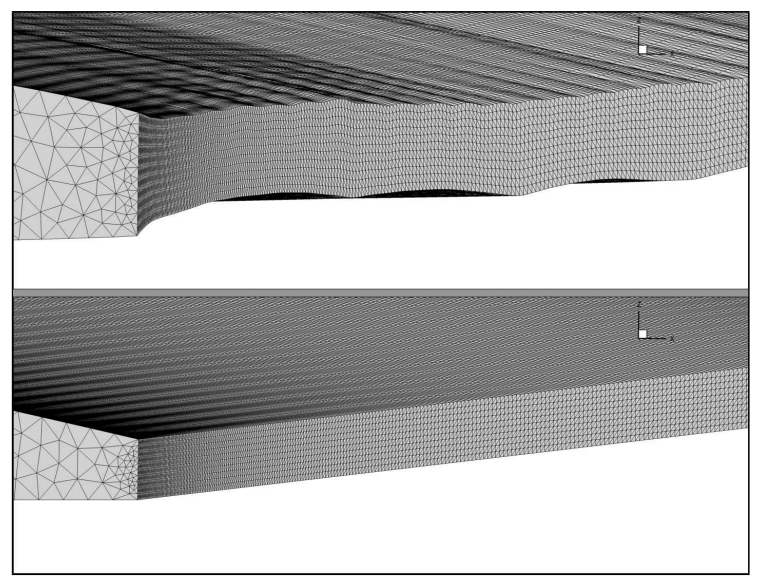

(b) Trailing Edge

Figure 5.7: Differences in the mesh topology between scan and ideal geometries

The scanned mesh had a fair amount of twist along the span, as shown in Figure 3.3. When the scanned mesh was imported, the only way to align it was along the estimated spar location similar to the DIC scans shown in Figure 3.2. 


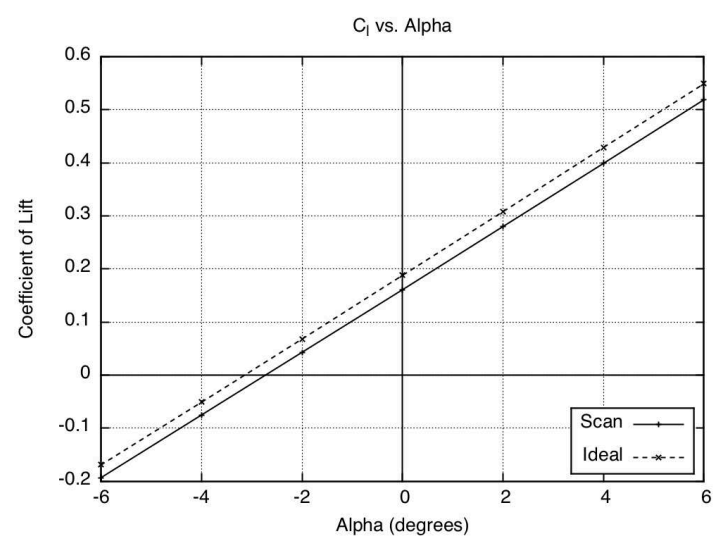

(a) Lift vs. Angle of Attack

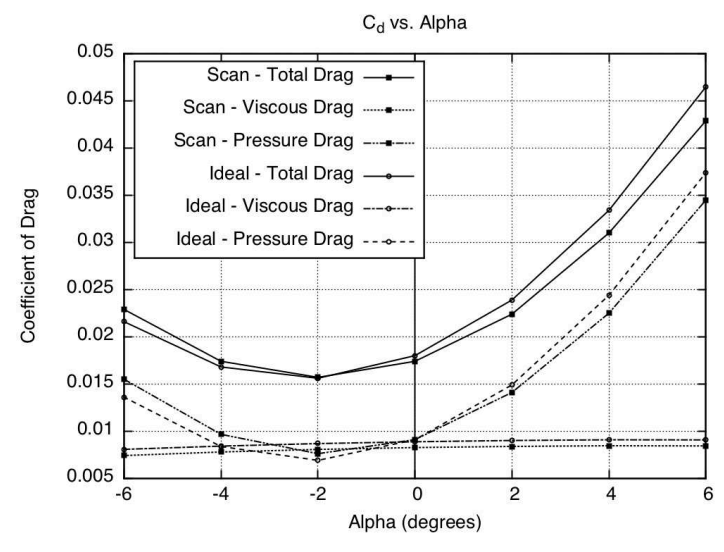

(b) Drag vs. Angle of Attack

Figure 5.8: Lift and Drag Comparisons - Scanned vs Ideal

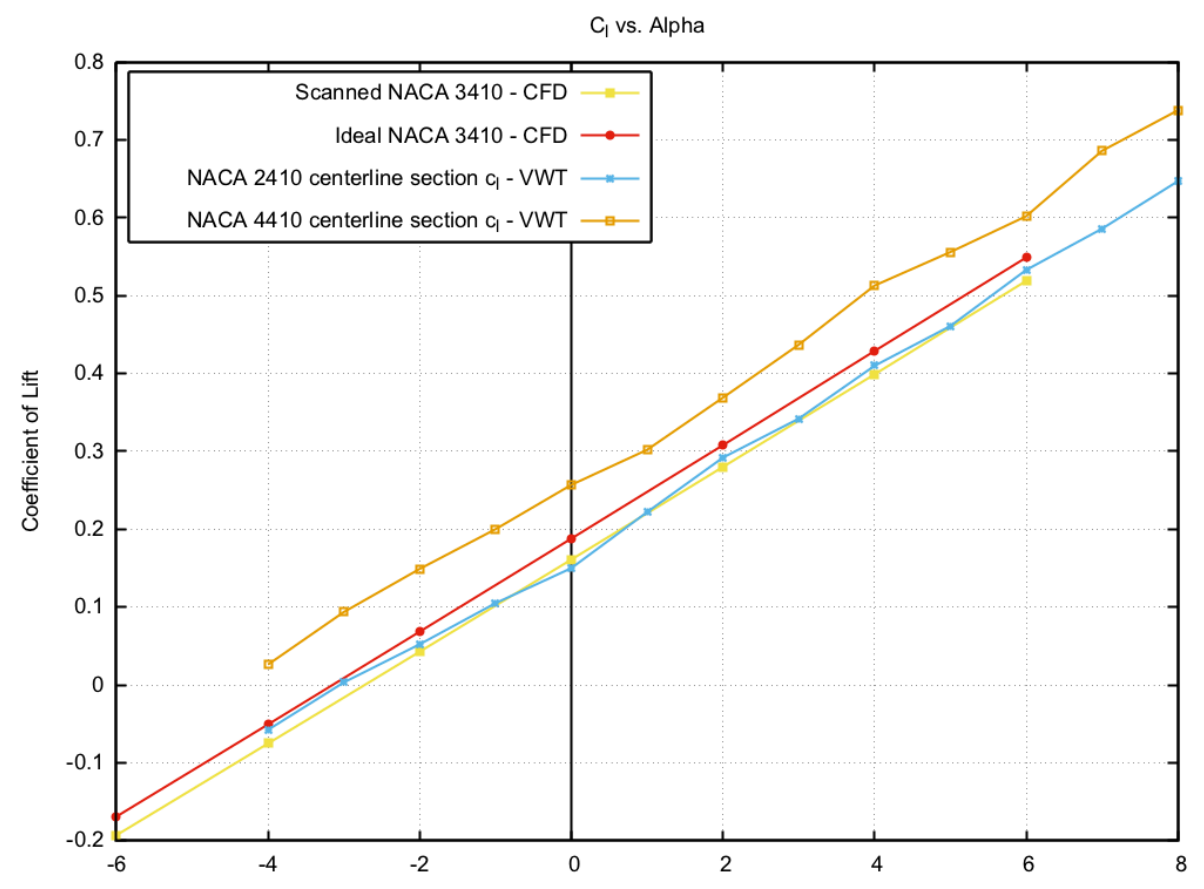

Figure 5.9: Scanned and Ideal Total Lift vs Experimental Sectional Lift

The results in Figure 5.8 show that the local twist changes the effective angle of attack. Figure 5.9 compares the results to the sectional lift coefficients taken from the pressure taps used during the wind tunnel test. The NACA 3410 configuration was never actually tested in the tunnel, so only the NACA 2410 and 4410 results are available and the best estimate would place the 3410 results 
between the two. Even though the centerline sectional lift is higher than the total lift, the trends shown by the scanned and ideal CFD align well. The scanned wing has a lower effective angle of attack compared to the ideal wing, and although it has a lower angle of attack throughout the entire sweep, it has a higher drag result for the negative angles. It is unlikely that the true results of the surface imperfections could be obtained using RANS simulations, but the trends were still quite interesting.

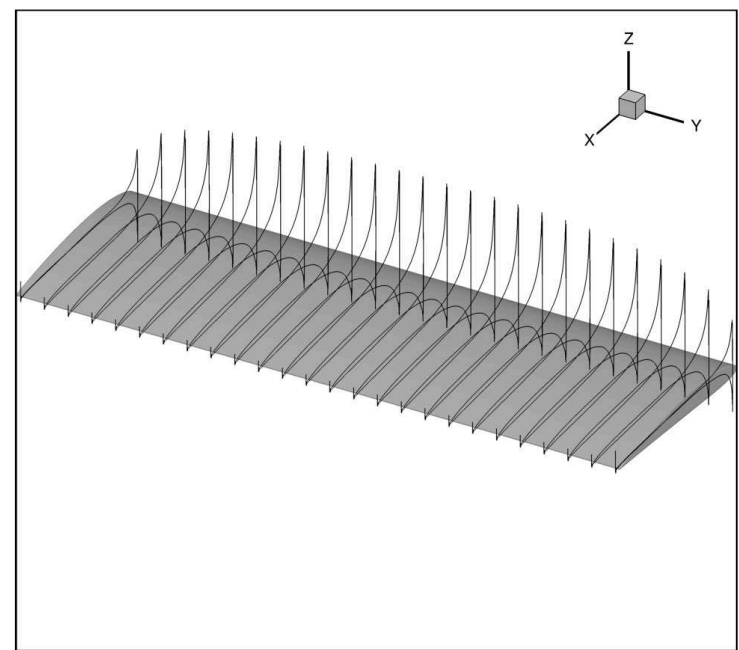

(a) Ideal NACA 3410

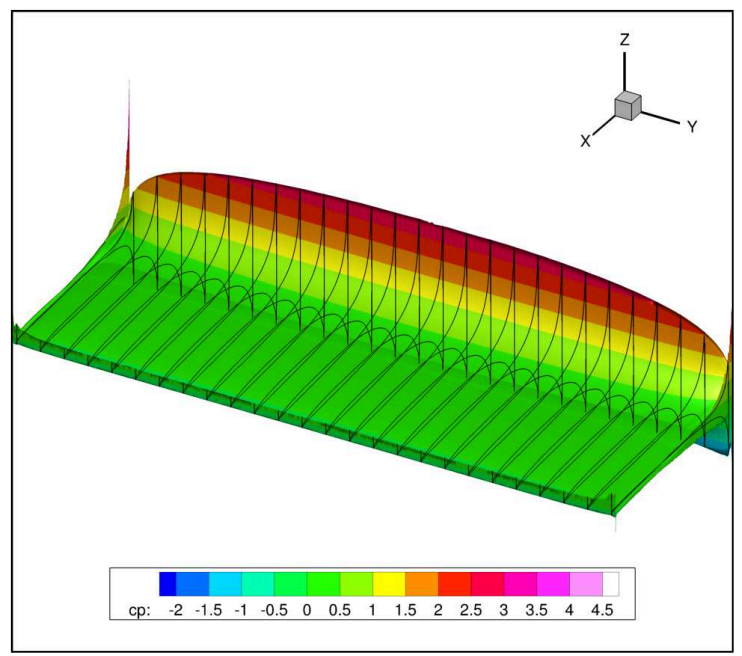

(c) Ideal NACA 3410

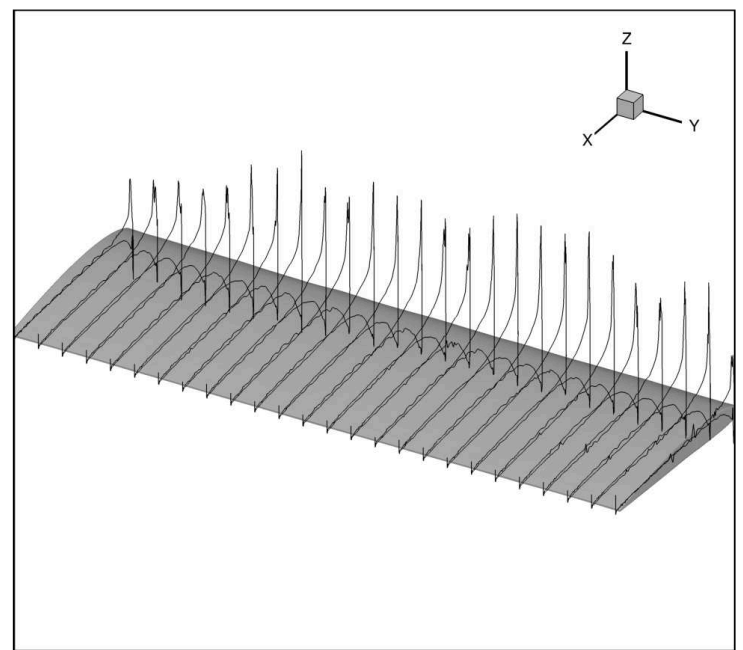

(b) Scanned NACA 3410

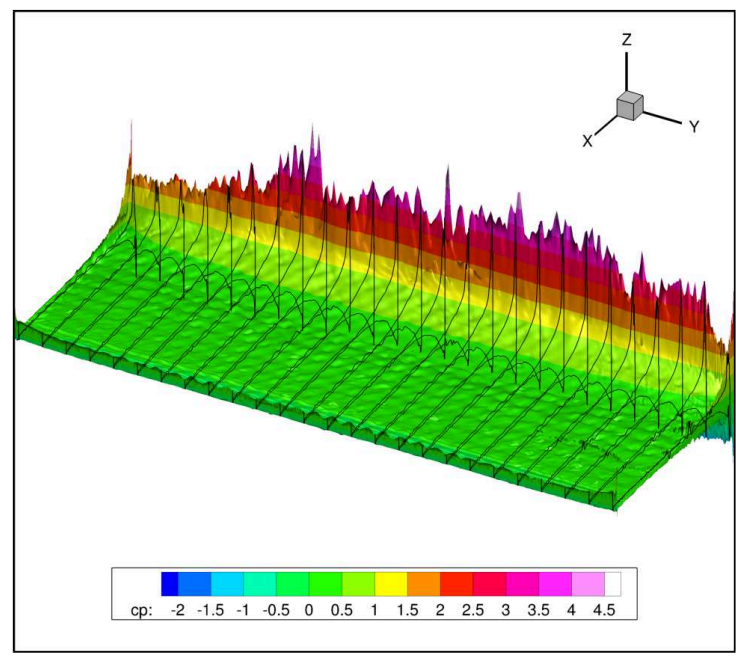

(d) Scanned NACA 3410

Figure 5.10: Effects of surface imperfections on sectional lift 
Figure 5.10 displays the sectional profiles of the pressure coefficient. The scanned mesh clearly has large variations due to the surface imperfections, but the overall integrated effect still has the same lift. While the overall lift at each angle of attack differs, as shown in Figure 5.8, the most likely culprit is the local twist variation along the span.

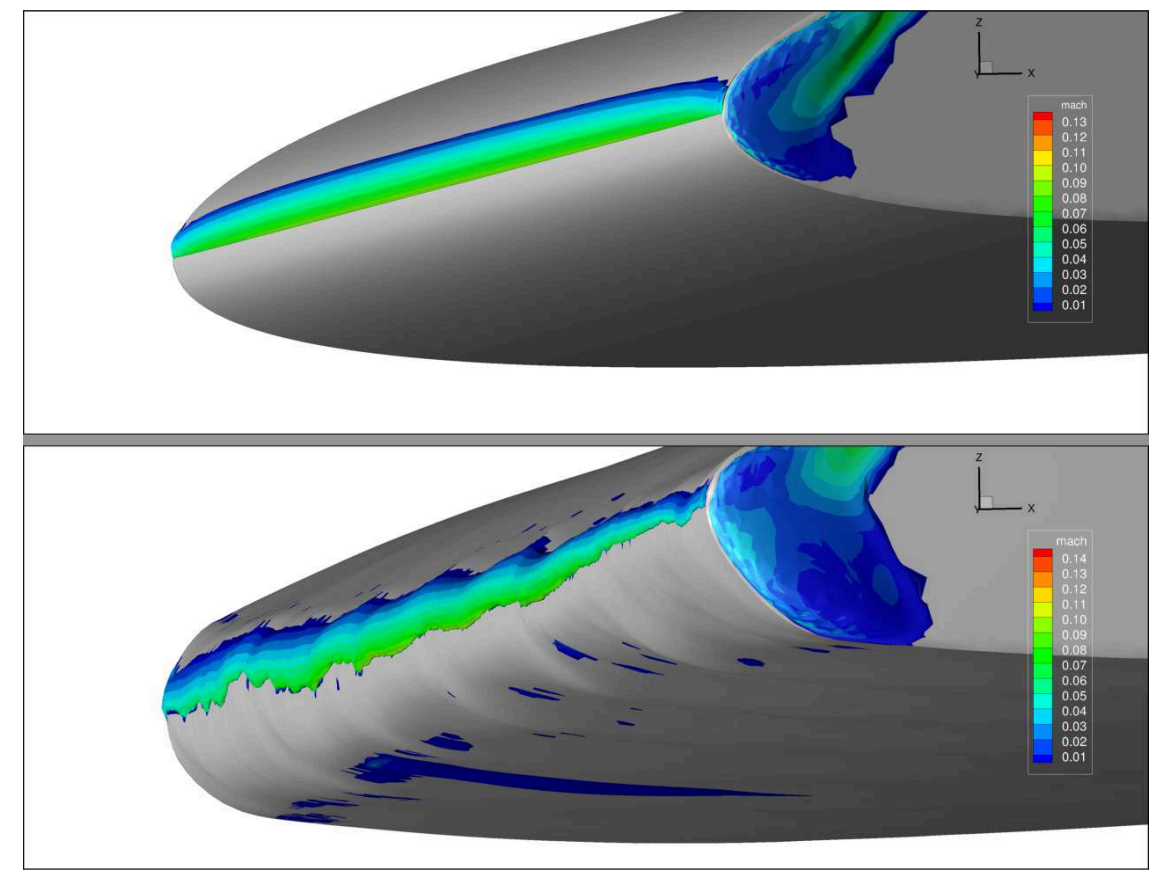

Figure 5.11: Iso-surfaces of Reversed Flow and Stagnation

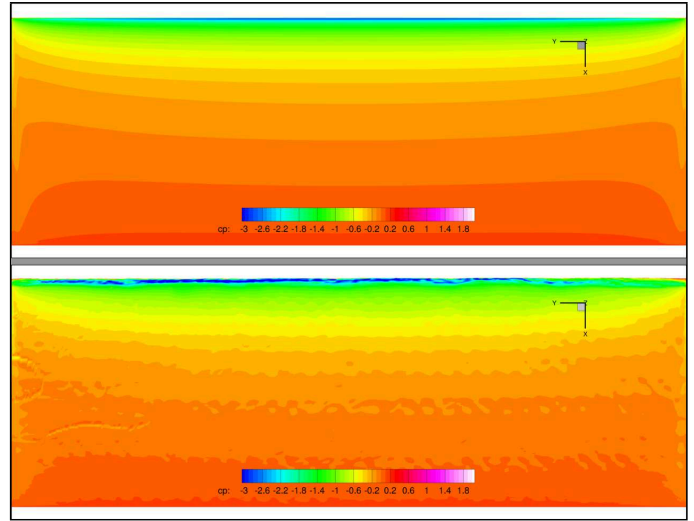

(a) Bottom Surface

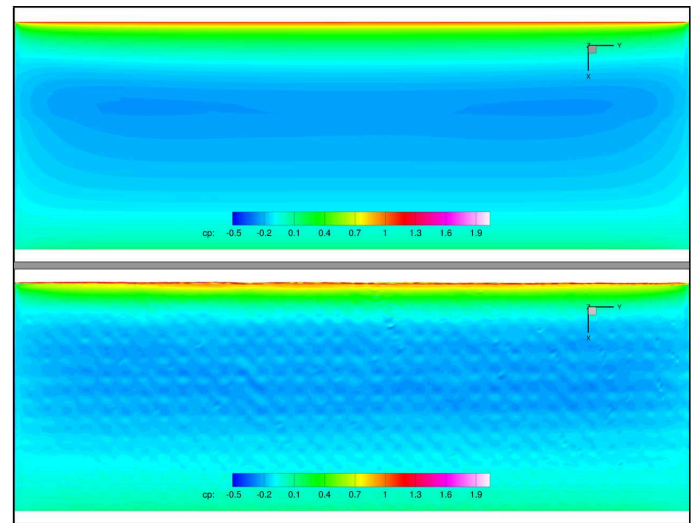

(b) Top Surface

Figure 5.12: Surface Pressure Distribution 
The localized separated flow, which can be seen in Figure 5.11, had significant impact on the drag. As it relates to the FSI analysis, the surface pressure distribution shown in Figure 5.12 compares the differences between the scanned and ideal wings. This pressure distribution directly impacted the way in which the VCCW deformed. The large variability in the coefficient of pressure at the leading edge, located at the top of the figures, signified that the leading edge region was considerably different. This, combined with the differences in material behavior, and even the inclusion of the leading edge, indicated that the results of the FSI analysis would be off - simply by the assumption of ideal geometry. The flow around the leading edge is shown in Figure 5.13 as a slice along the volume mesh at about half span with contours of the Mach number. This, in particular, showed how the large variation in leading edge shapes greatly affected the flow characteristics. In this instance, the bluntness of the leading edge caused the flow to separate and to reduce the pressure on the lower surface of the wing and the resulting deformation.

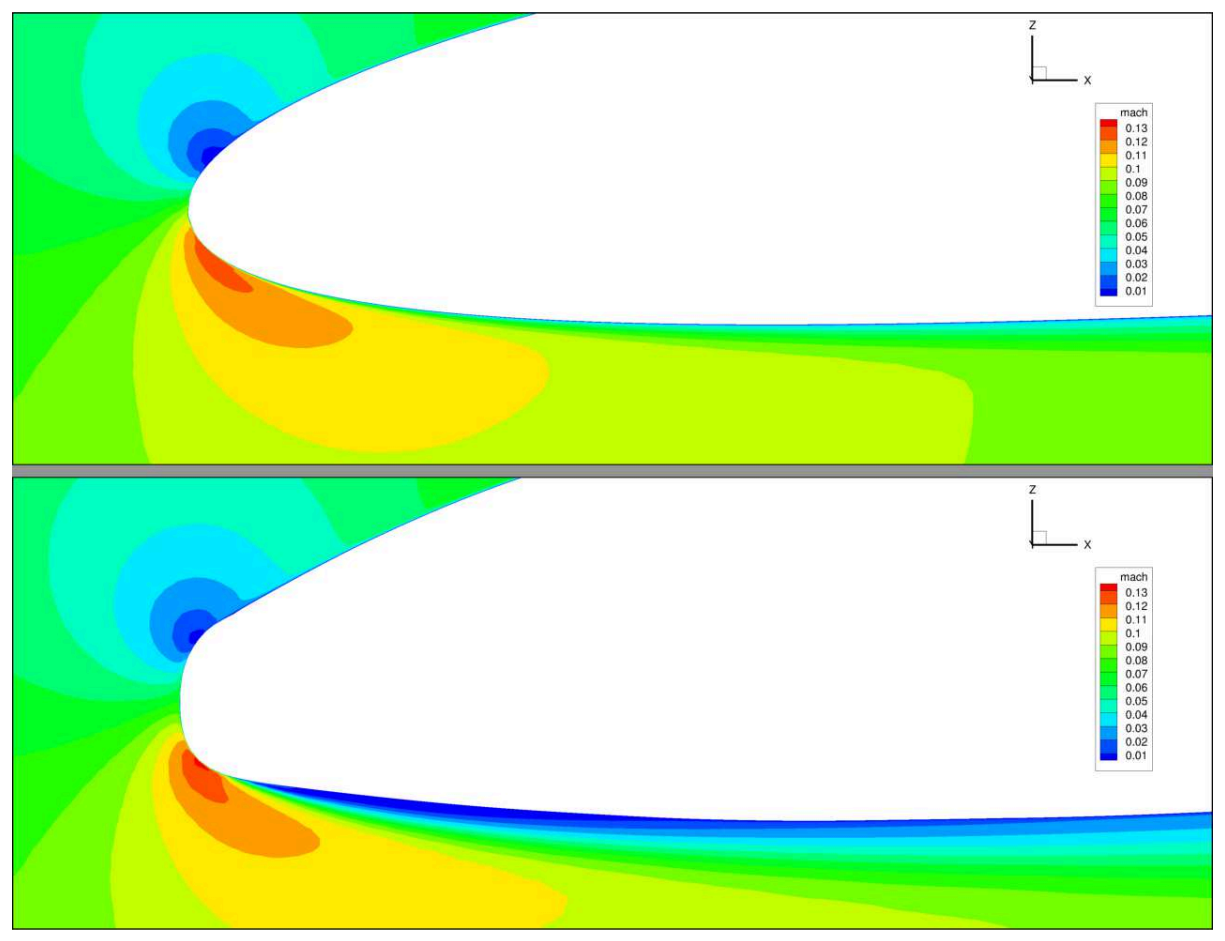

Figure 5.13: Leading edge shape variation between ideal and scanned geometry at the same spanwise location. Colored by Mach number 


\section{CHAPTER VI}

\section{MESH INTERPOLATION}

The mesh interpolation was done with a NASA Langley code called Discrete Data Transfer Between Dissimilar Meshes (DDTBDM), written by Jamesh Samareh. For partitioned FSI methods, the mesh interpolation stage is a critical step and, in this case, became the source of much frustration and error. Because CFD and FEA solvers have different needs, the meshes often look considerably different and have disparate resolutions. In this case, the CFD mesh was tetrahedral-based while the FEA mesh was dominated by quadrilaterals. However, while the resolutions of such meshes are often considerably different, the two meshes used were quite similar in resolution due to the amount of detail needed by the FEA solver to resolve the skin deformations.

Since DDTBDM could not natively accept Abaqus format, the solution was imported as two separate NASTRAN files; a model definition and a displacement file. These were then converted into a native format used by DDTBDM. The CFD mesh was also imported into the code and converted into the same format. Once both solutions were in native format, the code interpolated the displacements of the FEA solution onto the CFD mesh to create an updated and displaced CFD mesh. As stated previously, both the CFD and FEA meshes started out as a NACA 2410. A smooth-

ing function was then applied to the mapped mesh to untangle any cells and smooth out jagged edges created by the interpolation. 
The overall data transfer and work flow in DDTBDM is accomplished in three steps:

1. Find the nearest source element for every target point

2. Calculate the mapping coefficients for every target point and its corresponding (nearest) source element

3. Interpolate/inject from the source element to the corresponding target point

The major limiting factor in this analysis arose when the FEA mesh without leading edge material was interpolated onto the watertight CFD mesh. DDTBDM did a reasonable job for cases with no leading edge that had small cambers, which is why results are only shown for the NACA 2410 and 4410 cases. The higher camber cases were not included because the meshes were unusable, as shown in Figure 6.1. In the figure, the red mesh represents the original FEA mesh without the leading edge material and the yellow represents the area where the mesh interpolation results differed. As shown in the figure, it became quite obvious where the leading edge curvature deviated from the desired shape. Because there were no displacement definitions for the leading edge region, they were not deformed. The smoothing function was not enough to obtain an acceptable result.

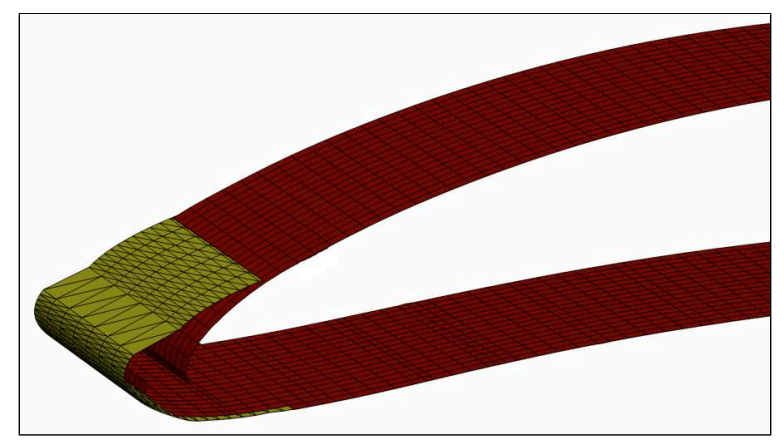

Figure 6.1: Leading Edge Interpolation Error 
Though there were numerous attempts to interpolate the large camber cases, DDTBDM just could not handle the large gap at the leading edge. The use of an intermediate mesh was also tried but was never successful. The thought behind this attempt was to interpolate the FEA solution for a large camber, e.g., the NACA 8410, onto a separately generated NACA 8410 mesh, which would then have the proper leading edge curvature. The intent was to then have a better estimate for the leading edge curvature mapped onto the FEA solution. Once the intermediate mesh had the FEA deformed shape mapped onto it, which would have included the leading edge curvature, it could then be mapped onto the final CFD mesh. Unfortunately, because of the way DDTBDM was designed, it simply maps nodal deformations from one mesh to the other. If no definition exists, it has to interpolate or smooth them. In the case of the VCCW, there was no definition for the nodes at the location of highest curvature. It would have been very useful, and potentially even good enough to solve this problem, if DDTBDM was able to simply project the deformed mesh onto a different mesh. Of all the locations in which accurate data was needed in order to interpolate properly, the leading edge was the most crucial. This likely would not have been an issue at the trailing edge or another location on the wing.

An alternative approach to fixing the leading edge problem, that was considered but deemed beyond the scope of the project with the remaining time available to implement it, was to create a spline along the leading edge to fill the gap with a smooth surface. Though spline creation would have been straightforward, working with the unstructured mesh would not have been. This approach would be greatly simplified if a structured mesh was used for the CFD surface mesh; in which case, one could have simply marched along the leading edge in a structured manner and project the nodes onto the surface created by the spline. However, an unstructured mesh requires connectivity data, and although it is included in the mesh definition, finding the nodes on the leading edge would not have been straightforward. 


\section{CHAPTER VII}

\section{FSI RESULTS}

The partitioned, loosely-coupled FSI analysis described in the previous chapters was applied to a portion of the experimental test matrix (see Table 3.1) as summarized in Table 7.1. The camber configurations and angles of attack chosen for analysis were influenced by the assumptions made for the FSI method. For example, the assumption of steady-state aerodynamics limited the analysis to small angles of attack, and the camber configurations were limited by material modeling of the leading edge, mesh interpolation failures, and lack of FEA convergence. Despite these limitations, the subset that was analyzed offered key insight into how the VCCW behaved under actuation and how FSI analysis can help understand the underlying physics for similar projects.

Table 7.1: FSI Analysis Matrix

\begin{tabular}{|c|c|c|c|c|}
\hline Camber & Alpha $\left(^{\circ}\right)$ & Configuration & CFD Mesh & Results \\
\hline \hline 2410 & $-4,0,6$ & No LE Material & Base, Medium & Included \\
\hline 2410 & $-4,0,6$ & Test Material \#3 & Base & Included \\
\hline 4410 & $-4,0,6$ & No LE Material & Base, Medium & Included \\
\hline 4410 & $-4,0,6$ & Test Material \#3 & Base & Included \\
\hline 6410 & $-4,0,6$ & No LE Material & Base & Mesh interpolation failed \\
\hline 6410 & $-4,0,6$ & Test Material \#3 & Base & FEA did not converge \\
\hline 8410 & $-4,0,6$ & No LE Material & Base & Mesh interpolation failed \\
\hline 8410 & $-4,0,6$ & Test Material \#3 & Base & FEA did not converge \\
\hline
\end{tabular}


The biggest challenge with simulating, testing, and analyzing the VCCW was the lack of knowledge about the specific shape of the model in the tunnel, regarding camber and angle of attack. Steps were taken to address this by providing profile shapes through detailed optical scans and displacement data from the digital image correlation. Analyzing the VCCW within the scope of the FSI simulation required the right data to compare to, in the form of DIC data, and the knowledge of proper solution convergence criteria, which was was only determined after studying this particular FSI problem. The initial estimate of FSI solution convergence was the point at which lift, drag, and structural deformations stopped changing significantly. Quantifying this criteria was achieved by recording the changes in aerodynamic and structural response data in the form of various norms. These norms and their change with respect to each prior FSI iteration is shown in Figure 7.1, where the displacement L2 norms and pressure coefficient distribution L2 norms are shown in pink and red, while the green and blue lines represent the change in the lift and drag coefficients, respectively. Figure 7.1(a) shows the effect of camber change in the residuals and Figure 7.1(b) shows the comparison between $0^{\circ}$ and $-4^{\circ}$ angle of attack. Note the influence of camber on the rate of convergence. The FEA solver must deflect the initial shape to the desired shape before the updated CFD solution can be run. This is reflected in the overall FSI convergence history; the rate of convergence is delayed because of the higher level of deformation caused by the camber change. This behavior is similar to the CFD convergence history detailed in Section V. 


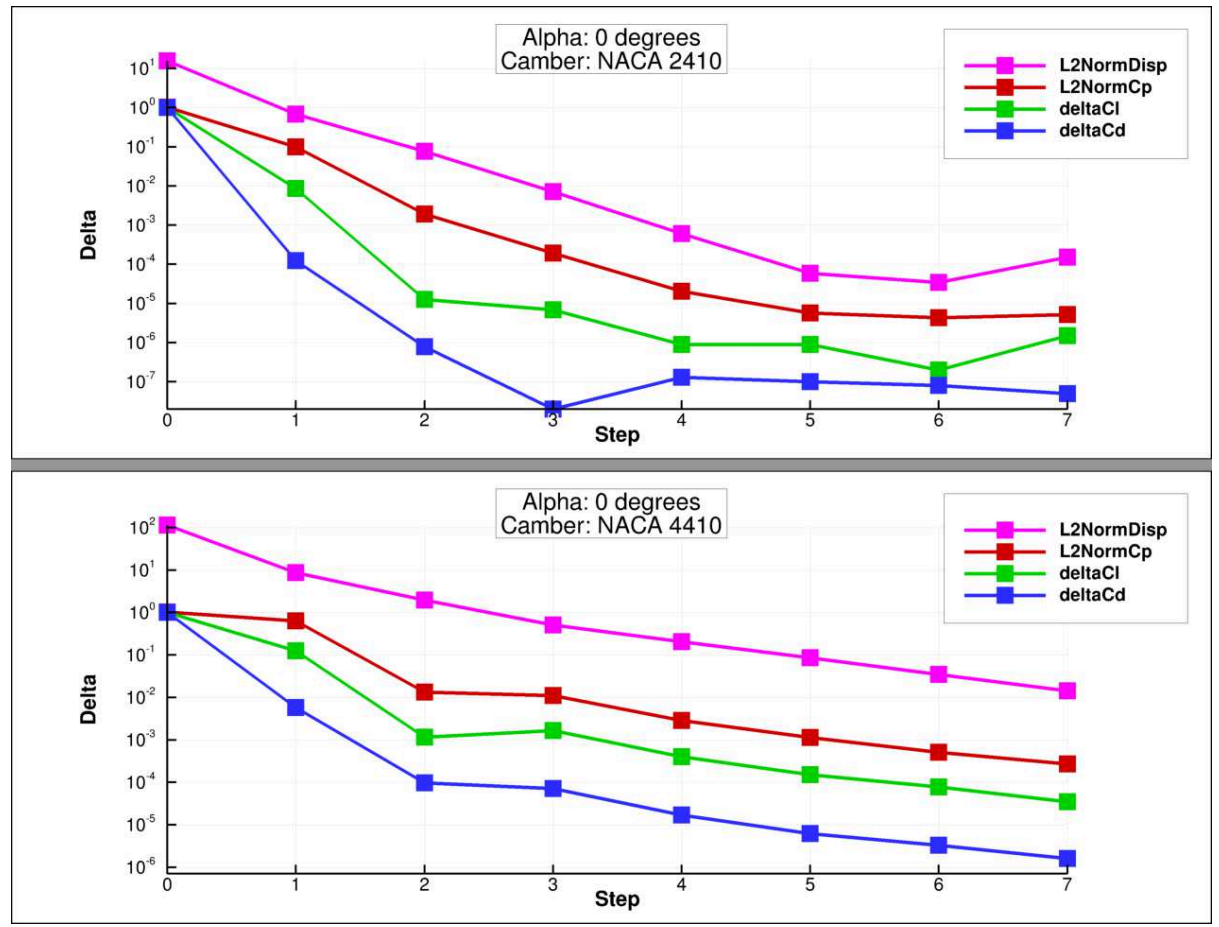

(a) Influence of camber
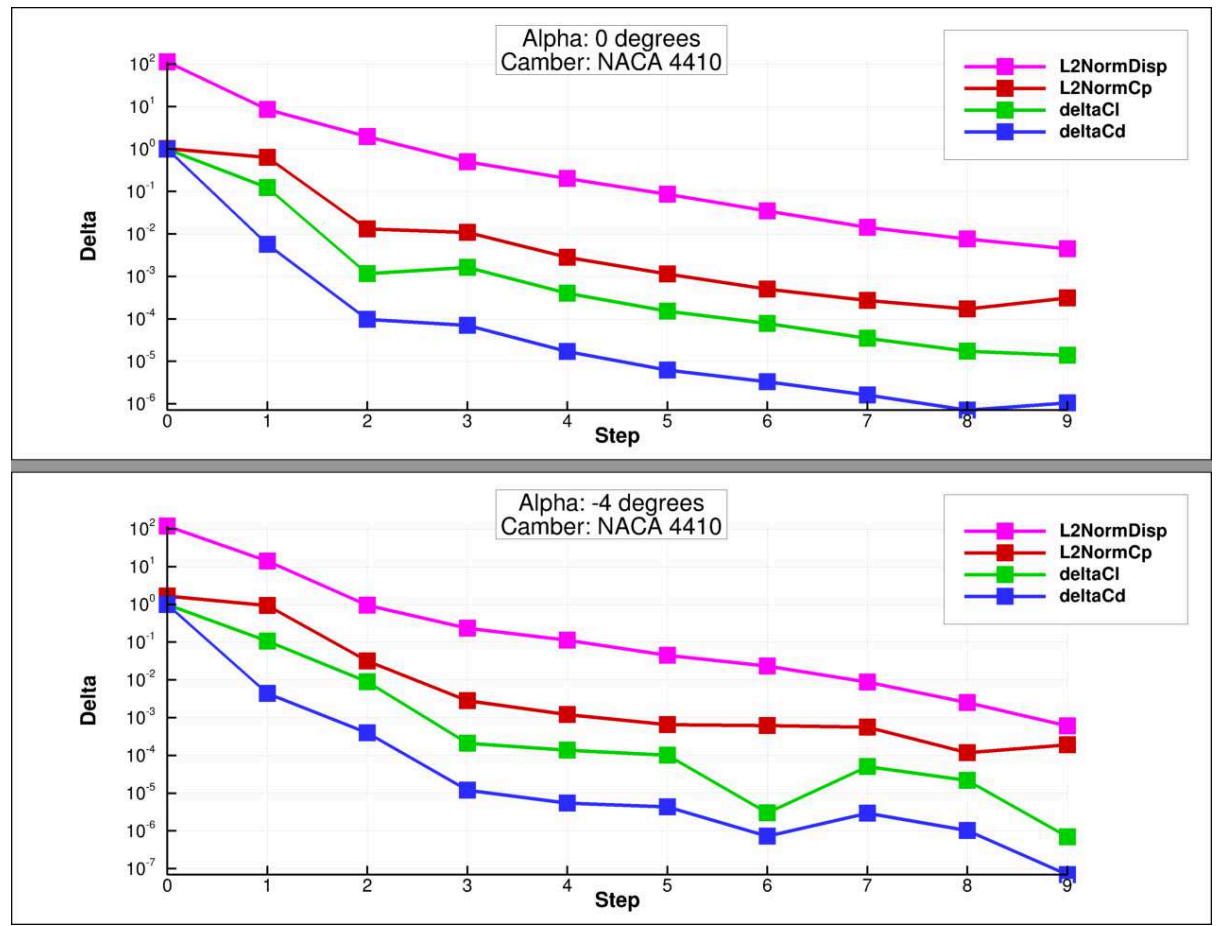

(b) Influence of alpha

Figure 7.1: FSI convergence history for the NACA 2410 and NACA 4410 


\subsection{DIC Comparison}

The general behavior of the wing as it varied camber and deformed under load is shown in Figure 7.2. Here, the upper surface deformation data, taken from the DIC experimental results, provided the best one-to-one comparisons to the experiment. Because the lift and drag data from the force couples built into the support structure was unusable, this was the primary comparison between the experiment and FSI analysis. The contour in the figure indicates the level of deformation normal to the surface due to aerodynamic loading and the subfigures compare the effect of angle of attack. The deformation caused by aerodynamics is shown, rather than the displacement amount needed to deflect to the desired camber. Each subfigure displays the camber progression in increasing levels, starting at a NACA 2410 and advancing to the NACA 8410 .

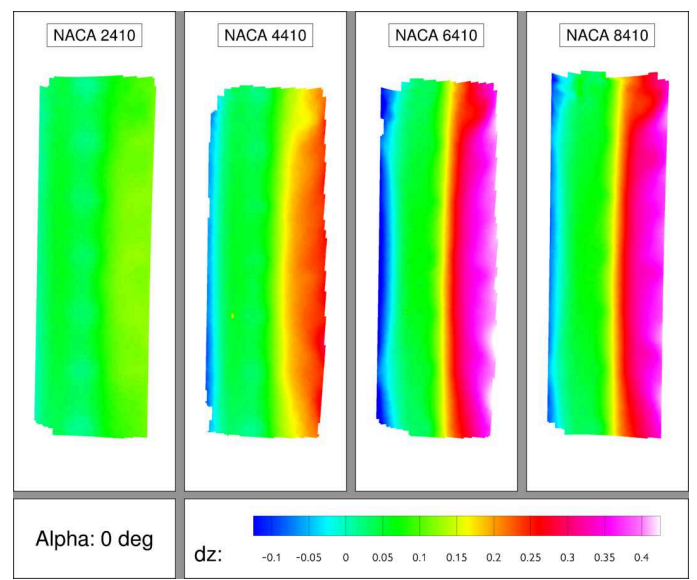

(a) Alpha $=0^{\circ}$

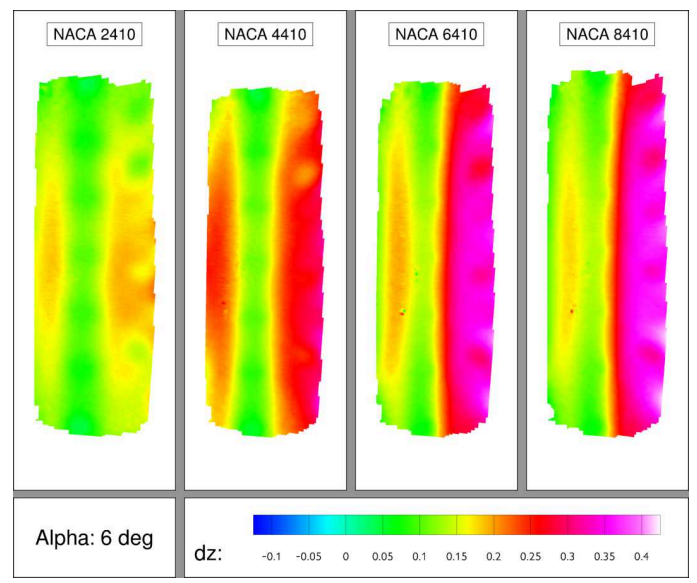

(b) Alpha $=6^{\circ}$

Figure 7.2: Deformation and Camber Progression

In both examples, the spar displacement was minimal and relatively uniform, and only deflected due to the lift distribution along the span, rather than as a result of actuation motion. For $0^{\circ}$ angle of attack, the deformation of the wing was fairly uniform along the span except for wing-tip vortex effects. These seemed to lessen the amount of deformation due to the reduction of lift in those areas. 
At $6^{\circ}$, considerably more lift was generated compared to the $0^{\circ}$ example, and span-wise variation in deformation became more visible. As the camber increased, the amount of deformation increased even though a larger amount of actuation deflection was used to achieve the higher camber. While actuating the wing would seem to stiffen the structure, at higher angles of attack the larger amount of lift caused increased deformation which overcame the deflection. Though not studied directly in this project, there came a point where the input actuation was not strong enough to overcome the aerodynamic loading. In general, the aerodynamic forces tended to reduce the camber of the wing. Ideally, a compliant wing would need the ability to deflect to multiple configurations, while still holding those positions without significant deformation, despite the increase in pressure. Interestingly enough, the NACA 8410 at $6^{\circ}$ seemed to have less deformation than the NACA 6410 . This seemed to be either a result of loss of lift due to separation or transitional flow effects, or that the leading edge droop caused by the camber was more aligned with the flow and experienced less of an adverse local pressure gradient. Here, the wingtip effects were less evident in the deformation response compared to $0^{\circ}$. Similar deformation behavior is produced at $-4^{\circ}$ and is shown in Appendix B.

Due to the assumptions made and the discrepancy between the simulation and experiment, the FSI displacements matched in trend rather than absolute value. Within the analysis, the general behavior of the deformation followed the experimental trends; their similarities are shown in the next series of figures. The graphs in Figure 7.3 show a span-wise slice from the wing at $96 \%$ chord comparing the effect of angle of attack. These illustrate the progression of the trailing edge deflection, at the NACA 4410 camber, as the FSI simulation marched toward a final solution, and are coded by color, from black to blue. The first and largest deflection came when the lift distribution was updated from the initial NACA 2410 camber to the deformed NACA 4410. The difference in 
angle of attack is shown in the lift and the resulting trailing edge deflection. The case at $-4^{\circ}$ is closer to the zero lift angle and thus has less deflection.

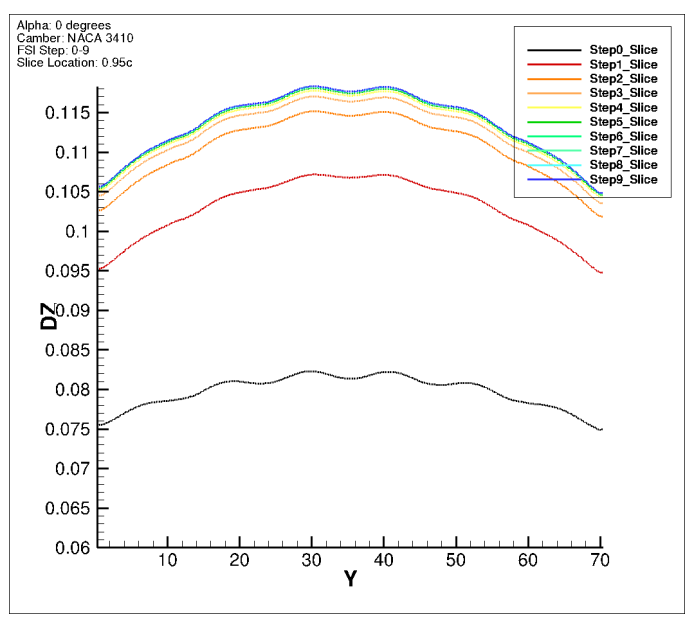

(a) NACA 4410, Alpha $=0$, Span-wise slice at $.95 \mathrm{c}$

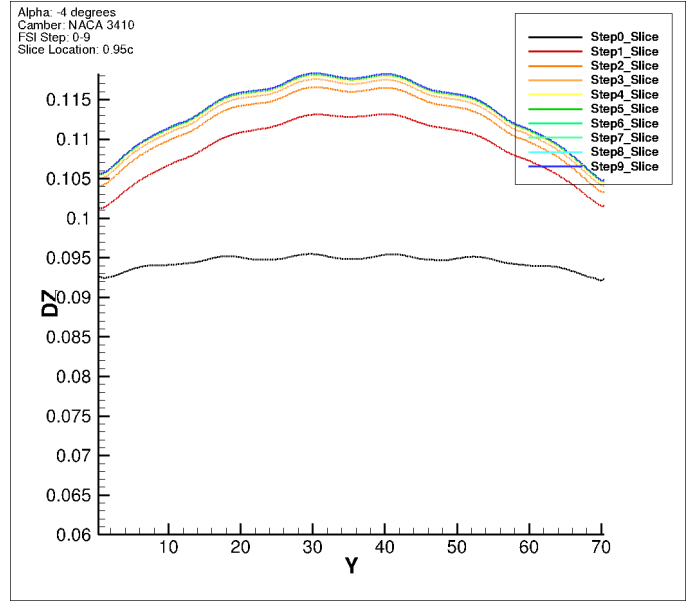

(b) NACA 4410, Alpha $=-4$, Span-wise slice at $.95 \mathrm{c}$

Figure 7.3: FSI Trailing Edge Deflection Progression

Figure 7.4 compares the deformation of the NACA 4410 for the FSI and DIC results at $62.5 \%$ and $80 \%$ percent chord. The twin y-axis is used to demonstrate that the magnitudes do not match, but the behavior is similar. The overall deformation for this case, and most others, under-predicts when compared to the experiment. This could be the result of not including the fixture deflection in the FEA model. Some of the differences in rib location, which show as peaks and valleys in the deformation data, were due to alignment issues with the experimental data. During the experiment, the amount of compliant mechanism actuation varied slightly between each rib, as shown in Figure 3.3, whereas the FSI analysis assumed uniform actuation throughout. Note in Figure 7.4 that the FSI results contain both the upper and lower surface displacements, but the experimental results were only of the top surface. The deformation trends were similar, specifically the behavior at and between the ribs, along the centerline of the span. The deformation was considerably different at the tips due to the wing-tip vortex interaction and support structure interference effects which were not 
included in the analysis. Additional fidelity and accuracy would have been possible if the actuation of the compliant mechanism could have been directly matched to the input used during testing.

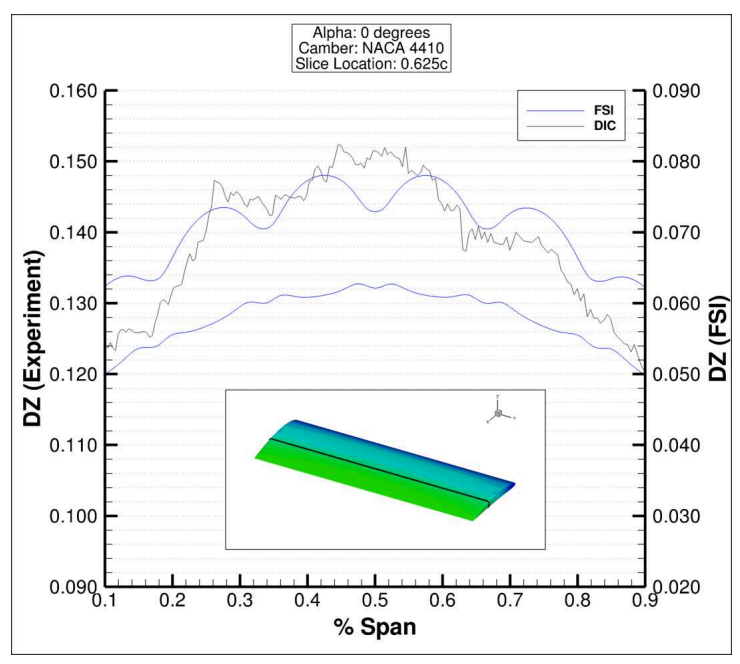

(a) NACA 4410: Alpha $=0$, Span-wise slice at $.625 \mathrm{c}$

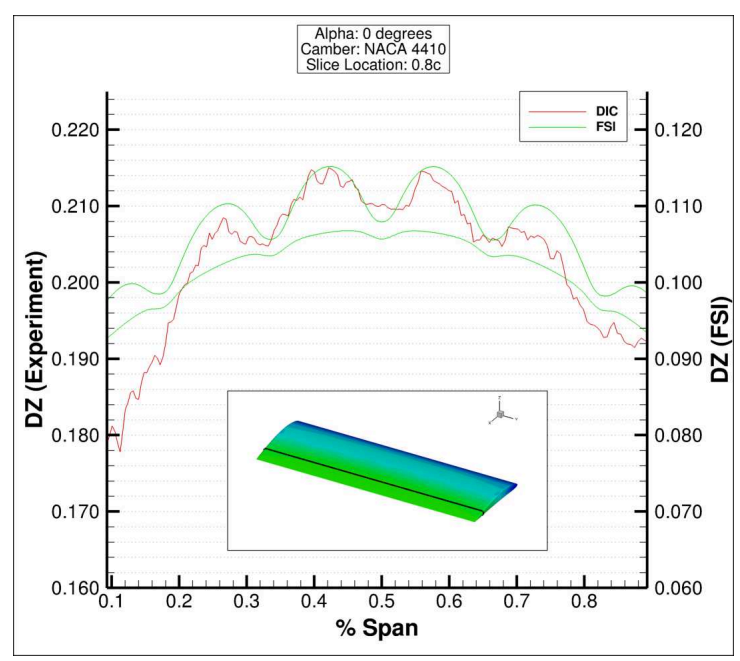

(b) NACA 4410: Alpha $=0$, Span-wise slice at $.8 \mathrm{c}$

Figure 7.4: FSI vs. DIC Results - Displacement Slices

The coupled nature of the physical problem meant that the CFD and FEA configurations on their own, as well as the effect of the coupled model as a whole, impacted the final result.

Figure 7.5(a) shows how the CFD mesh resolution changes the FSI results for the same camber (NACA 4410), angle of attack $\left(-4^{\circ}\right)$, and FEA mesh, with two different CFD mesh resolutions (baseline and medium).

The medium resolution mesh did a better job capturing the finite wing effects as well as the overall lift distribution, as shown by the increased deflection of the trailing edge. As mentioned in the FEA modeling section, the choice of material models was a point of great difficulty and one that still needs to be further investigated, due to the complexity of the materials involved. Multiple test specimens were taken of the leading edge material, but the variation between them yielded a highly non-linear material that was difficult to model in Abaqus. Figure 7.5(b) shows how much the material choice affected the end result. Here, the leading edge material determined the amount of 
displacement, even at the trailing edge, due to the single continuous skin that the prototype was built with. When the leading material was included in the FEA model, the deformation of the leading edge affected the behavior of the trailing edge. When the material was not included, the trailing edge did experience as much displacement, and there was more span-wise variation of the upper surface deflection forward of the spar. The skin along the LE of the mesh also displaced slightly more because there was no material impediment as compared to the case with the leading edge material included.

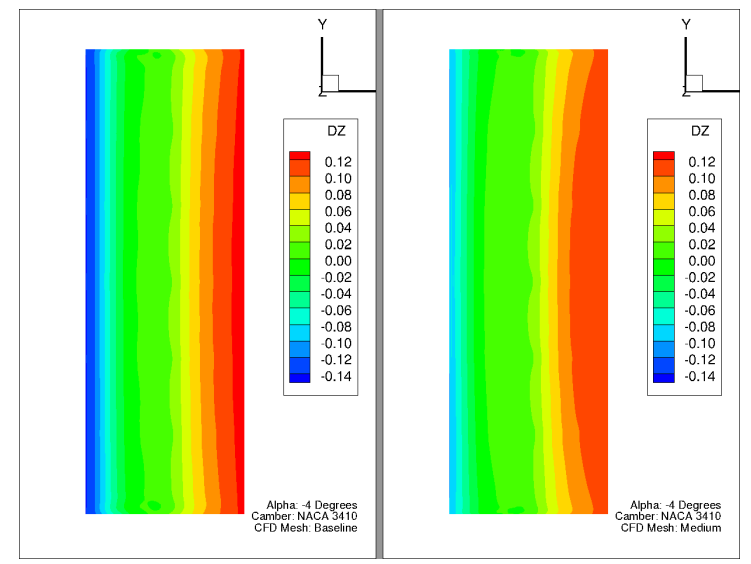

(a) CFD Mesh Effects (Left: Baseline, Right: Medium)

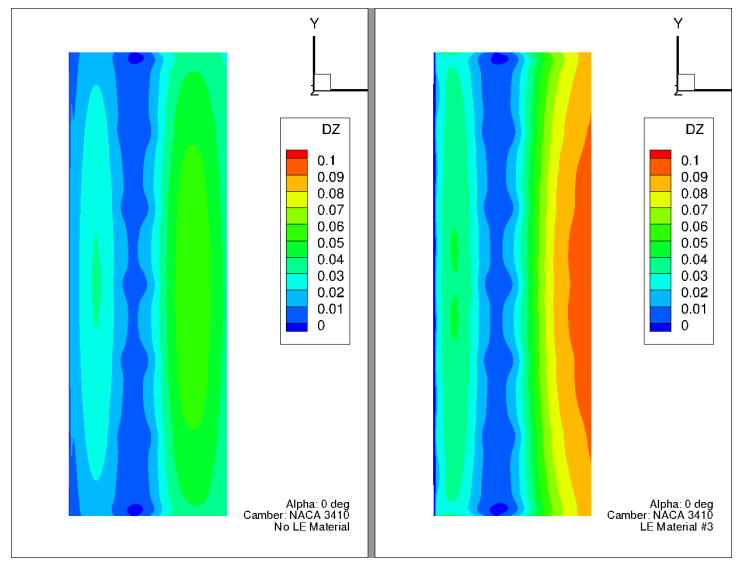

(b) FEA LE Material Effects (L: No LE, R: Est. Material)

Figure 7.5: CFD/FEA configuration effects

The next series of charts, Figures 7.6 through 7.8 directly compare the FSI and DIC results. In this series, the subfigures on the left hand side (a, c, and e), show the cases compared with separate contour levels to show behavior trends rather than matched magnitudes. The subfigures on the right (b, d, and f) use matching contour levels to compare absolute magnitudes of displacement. These subfigures are then split up into three categories; the first two show the baseline and medium CFD mesh with no leading edge material, and the third shows the baseline CFD mesh with an estimated leading edge material. Some of these results were washed out in the contour when compared to the experiment because their magnitudes were much lower than the experiment. Note that the 
displacements shown in the figures are in inches, and that none of the displacement magnitudes exceed one inch in either the FSI or the experiment. Figure 7.6, which shows the comparison of the NACA 2410 cases, includes the material estimate used in Abaqus. The estimated material model performed much closer to the experiment, because it captured the general spar interaction and rib behavior much better than those with no leading edge material, but only for this case. 

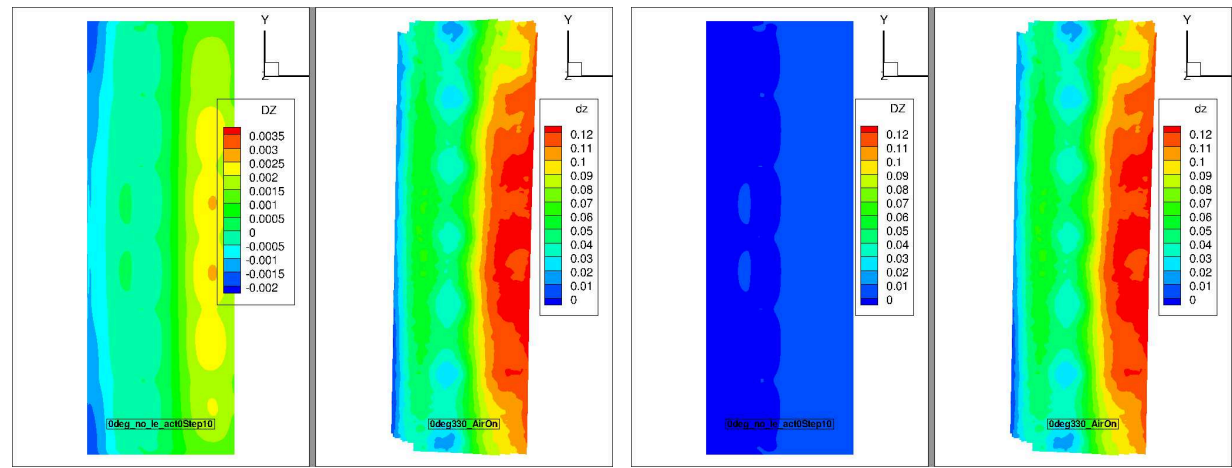

(a) Baseline Resolution: Separate Contour (Left: FSI, Right: DIC)

(b) Baseline Resolution: Matched Contour (Left: FSI, Right: DIC)
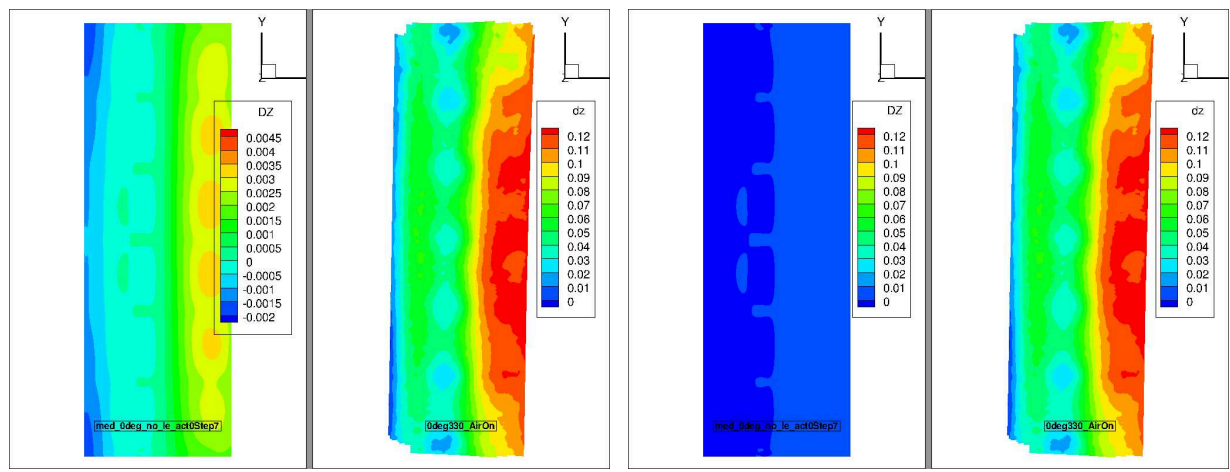

(c) Medium Resolution: Separate Contour (Left: FSI, Right: DIC)

(d) Medium Resolution: Matched Contour (Left: FSI, Right: DIC)

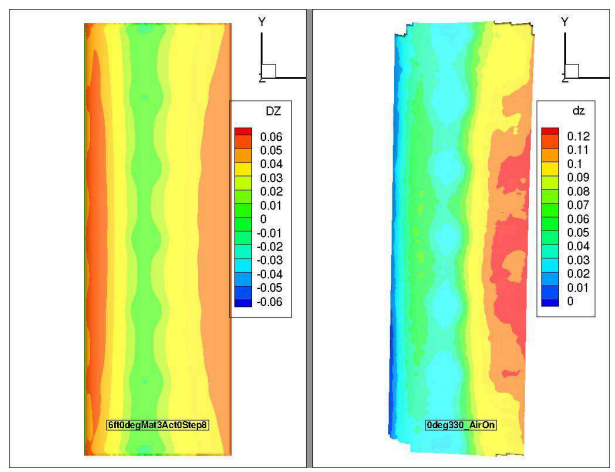

(e) Material 3: Separate Contour (Left: FSI, Right: DIC)

(f) Material 3: Matched Contour (Left: FSI, Right: DIC)

Figure 7.6: NACA 2410 Target Camber, Alpha $=0^{\circ}$ 


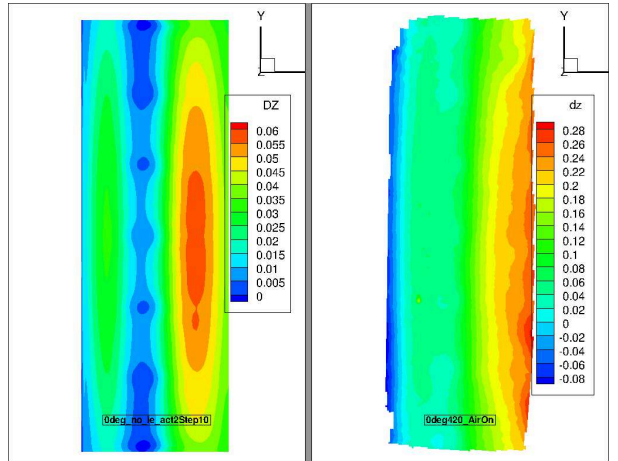

(a) Baseline Resolution: Separate Contour (Left: FSI, Right: DIC)

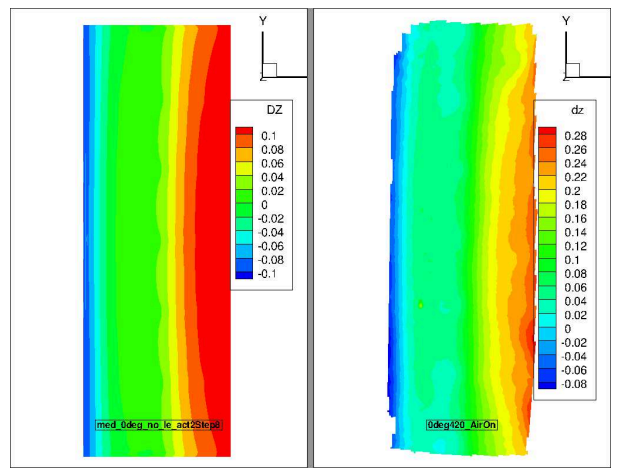

(c) Medium Resolution: Separate Contour (Left: FSI, Right: DIC)

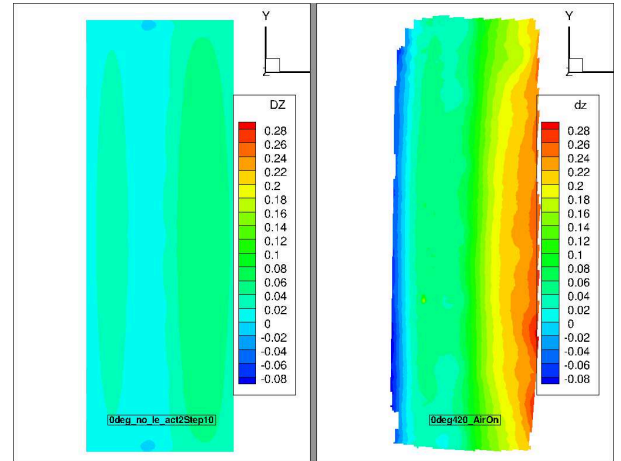

(b) Baseline Resolution: Matched Contour (Left: FSI, Right: DIC)

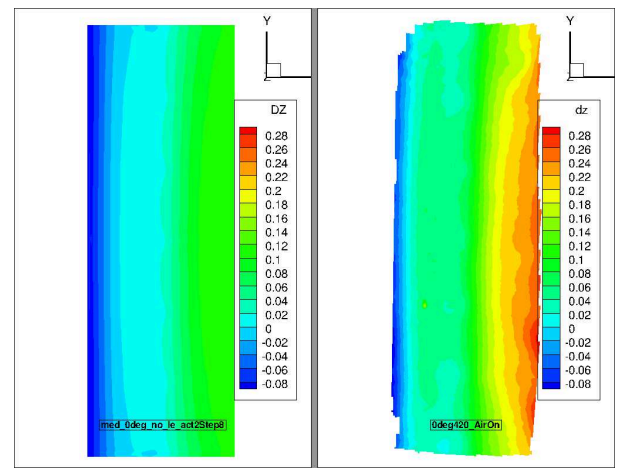

(d) Medium Resolution: Matched Contour (Left: FSI, Right: DIC)

Figure 7.7: NACA 4410 Target Camber, Alpha $=0^{\circ}$

For the NACA 4410 at $0^{\circ}$, the estimate material did not work due to convergence issues in Abaqus. Here, the cases without leading edge material performed much closer to the experiment. However, they did not do as well at predicting the slight bending of the spar in either the baseline or medium CFD mesh. This is likely due to the lack of support beam simulation in both the FEA and CFD meshes. Both cases captured the general behavior of the leading and trailing edges, but there was considerable difference and improvement in the results when the medium resolution CFD mesh was used, as shown in Figure 7.7(c) and 7.7(d). 


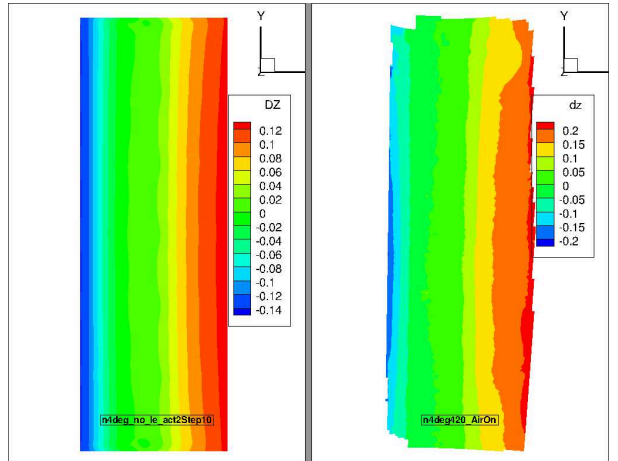

(a) Baseline Resolution: Separate Contour (Left: FSI, Right: DIC)

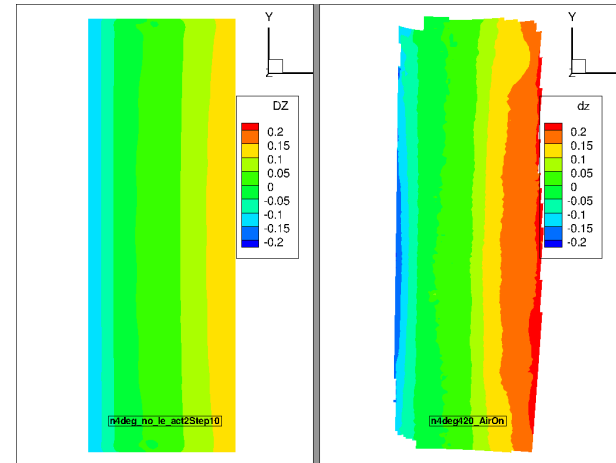

(b) Baseline Resolution: Matched Contour (Left: FSI, Right: DIC)

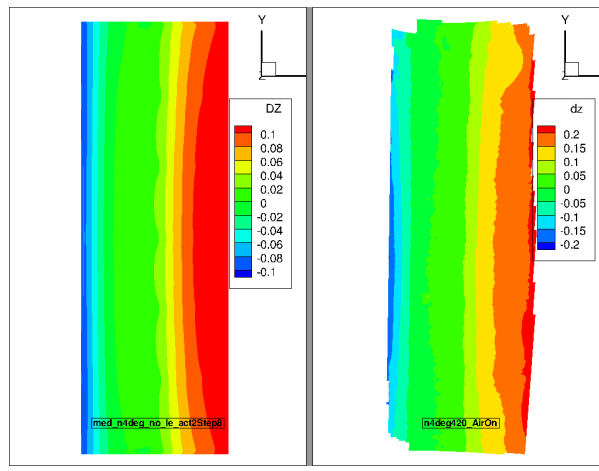

(c) Medium Resolution: Separate Contour (Left: FSI, Right: DIC)

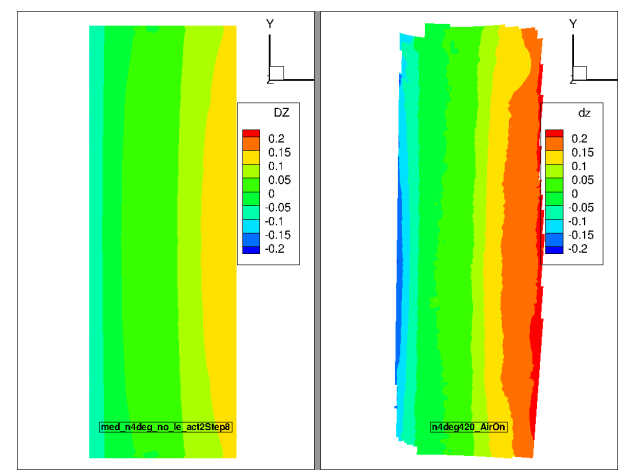

(d) Medium Resolution: Matched Contour (Left: FSI, Right: DIC)

Figure 7.8: NACA 4410 Target Camber, Alpha $=-4^{\circ}$

Figure 7.8 showcases the results from the NACA 4410 target camber at $-4^{\circ}$. The estimate material case is also not included for this result due to convergence issues within Abaqus. Interestingly, this case performed much better compared to other camber and angles of attack. The medium CFD mesh captured more of the lift distribution behavior when compared to the baseline, but neither mesh captured the wing tip effects shown in the DIC surfaces due to assumptions of steady-state flow and the use of RANS turbulence models. The $-4^{\circ}$ angle was closest to the zero-lift angle for the NACA 4410 centerline lift coefficient, as demonstrated by the lift curves shown in Figure 5.9, and may explain why the FSI results are closer. If FUN3D was not capturing certain physics at the lifting angles, the results may have been lessened near the zero-lift alpha. 
Overall, the FSI results trended towards under-predicting the deflections, but still captured similar behavior. The VCCW tended to deflect in the direction of the lift, as a result of the aerodynamic forces, which effectively reduces the amount of camber. The CFD resolution study showed higher lift as the resolution increased, and the CFD mesh effects in Figure 7.5(a) showed an increase in deflections as well, due to the higher lift. Figures 7.6 through 7.8 as well as the remainder of results in Appendix B indicate that the FSI consistently under-predicted the trailing edge deflection in all cases. 


\section{CHAPTER VIII}

\section{CONCLUSION}

This thesis presented results for a loosely-coupled fluid-structure interaction (FSI) of a flexible wing, using FUN3D to compute the aerodynamic flow-field and Abaqus to calculate the structural deformation. NASA Langley provides a general 3D algorithm to interpolate between dissimilar meshes, which was used here to map pressures and displacements between the aerodynamic and structural codes. This method was applied to the AFRL-developed "Variable Camber Compliant Wing" (VCCW), which is an adaptable wing designed to target airfoil shapes between a NACA 2410 and 8410 . The VCCW was tested in the vertical wind tunnel facility at WPAFB. These tests provided experimental data in the form of static pressure tap data, digital image correlation, and oil flow visualization. Optical scans also provided to obtain detailed information about the shape of the wing at each camber configuration. These scans were used to match the FEA profiles with those encountered during the experiment.

The FEA model of the VCCW that was created during the design phase of the project was modified to facilitate communication between multiple codes for the FSI analysis. The VCCW prototype was constructed with non-homogeneous composite materials that are difficult to model computationally. Even though considerable research was done to investigate computational material models of the leading edge of the VCCW, there is still much room for improvement. This was one of two limiting factors in the FSI analysis and subsequently reduced the available camber configurations to 
only the NACA 2410 and 4410. A material model comparison was completed to compare the difference between an estimate material model versus eliminating the leading edge material altogether.

A CFD analysis was done on multiple configurations of the VCCW to investigate the aerodynamic effects of the support structure and non-uniform construction of the prototype. The results concluded that these effects were significant and cannot be neglected in future research. Because the FSI simulation assumed ideal conditions, the solutions of lift and drag did not directly match the experiment, where the actual geometry was far from ideal. Boundary layer tripping and flow separation caused by the skin of the VCCW significantly changed the local pressure distributions on the surface, which ultimately changed the behavior of the deformation.

For partitioned FSI methods, the mesh interpolation stage is a critical step, and in this case, was the source of much frustration and error. The second limiting factor in this analysis arose when the FEA mesh versions without leading edge material were interpolated onto a watertight CFD mesh. Without any nodal displacement definitions at the location of highest curvature and displacement, the interpolation code was unable to resolve sufficient topology that suitably matched the experiment. This made large cambers, like the NACA 6410 and 8410, impossible to simulate. Though there were numerous attempts to interpolate the large camber cases, ultimately it was determined that the code could not handle the large gap at the leading edge.

The combined solutions from the FEA, CFD, and mesh interpolation solvers created FSI results that were similar in trend to the experiment, but consistently under-predicted the deformations of the VCCW. The peak and valley behavior of the skin interacting with the ribs was captured well but effects at the spar and wing tips were not captured in all cases. The configurations without the leading edge material were more successful when the wing was actuated. The only case in which including the material yielded better results was the un-actuated NACA 2410. All of this indicates that the material model is the essential piece of the analysis. Without a proper material model, 
only trends can be predicted. CFD typically performs well when predicting aerodynamics and FEA typically does well predicting structural deformations, so the solvers themselves are not at fault, nor is the coupling method. The VCCW did not encounter aeroelastic flutter during the test, or even significant deformation, since the displacements were, at most, on the order of one inch. The loosecoupling method would more likely be at fault in cases where the fluid and structure interaction were a dominant force, in arterial blood flow for example, but the VCCW was a relatively stiff structure and the flow velocity was only $26 \mathrm{~m} / \mathrm{s}$. If the same analysis used here were applied to a simpler model, with a well defined structure and flow field, the results would be much more conclusive overall.

\subsection{Future Work}

For future work, additional fidelity and accuracy could be achieved by adding the effects that were not included in this project. Aerodynamically, the wind tunnel and support structure has real impact that cannot be ignored. Time dependent effects like vortex shedding created by the wingtips and supporting structure will have an impact on the amount of lift and drag felt, particularly at higher angles of attack. FUN3D allows for the use of adjoint-based mesh adaptation, which would be simple to implement in the future, that would improve the quality of the mesh and accuracy of the solution. From the structural dynamics perspective, both the support structure and the weight of the wing have an impact on the bending of the spar. Including the support structure in the FEA analysis would be straightforward since the model has already been created and merged with the wing model. Researching and obtaining a better material model should be the primary focus of further research applied to an FSI analysis of the VCCW, since this had the largest detrimental effect on the entire project. If a more accurate material model could have been used, the FEA analysis would have been streamlined and all of the camber configurations would be available for analysis. 


\section{BIBLIOGRAPHY}

[1] J. Joo, C. Marks, and L. Zeintarski, “AFRL Variable Camber Compliant Wing - Design," in SciTech 2015, Kissimmee, FL, 2015.

[2] S. Miller, M. Rumpfkeil, and J. Joo, "Fluid-Structure Interaction of a Variable Camber Compliant Wing," in 53rd AIAA Aerospace Sciences Meeting, ser. AIAA SciTech. Kissimmee, FL: American Institute of Aeronautics and Astronautics, Jan. 2015.

[3] R. T. Biedron, J. M. Derlaga, P. A. Gnoffo, D. P. Hammond, W. T. Jones, B. Kleb, E. M. Lee-rausch, E. J. Nielsen, M. A. Park, C. L. Rumsey, J. L. Thomas, and W. A. Wood, FUN3D Manual: 12.5, nasa/tm20 ed. Hampton, VA: NASA Langley Research Center, 2014, no. September.

[4] J. Samareh and K. Bhatia, "A unified approach to modeling multidisciplinary interactions," in 8th Symposium on Multidisciplinary Analysis and Optimization, ser. Multidisciplinary Analysis Optimization Conferences. American Institute of Aeronautics and Astronautics, Sep. 2000. [Online]. Available: http://dx.doi.org/10.2514/6.2000-4704

[5] J. Samareh, "Discrete Data Transfer Technique for Fluid-Structure Interaction," in 18th AIAA Computational Fluid Dynamics Conference, ser. Fluid Dynamics and Co-located Conferences. American Institute of Aeronautics and Astronautics, Jun. 2007. [Online]. Available: http://dx.doi.org/10.2514/6.2007-4309

[6] R. Kamakoti and W. Shyy, "Fluidstructure interaction for aeroelastic applications," Progress in Aerospace Sciences, vol. 40, no. 8, pp. 535-558, Nov. 2004. [Online]. Available: http://linkinghub.elsevier.com/retrieve/pii/S0376042105000084

[7] M. Behr and T. Tezduyar, "Finite element solution strategies for large-scale flow simulations," 1994.

[8] A. Johnson and T. Tezduyar, "Mesh update strategies in parallel finite element computations of flow problems with moving boundaries and interfaces," 1994.

[9] A. Eken, M. Sahin, and A. Eken, "A Monolithic Approach for the Numerical Simulation of Fluid Structure Interaction Problems," in 43rd Fluid Dynamics Conference, ser. Fluid Dynamics and Co-located Conferences. American Institute of Aeronautics and Astronautics, Jun. 2013. [Online]. Available: http://dx.doi.org/10.2514/6.2013-3199 
[10] C. Farhat, P. Geuzaine, and G. Brown, "Application of a three-field nonlinear fluidstructure formulation to the prediction of the aeroelastic parameters of an F-16 fighter," Computers \& Fluids, vol. 32, no. 1, pp. 3-29, Jan. 2003. [Online]. Available: http://linkinghub.elsevier.com/retrieve/pii/S0045793001001049

[11] C. Farhat, K. G. van der Zee, and P. Geuzaine, "Provably second-order time-accurate looselycoupled solution algorithms for transient nonlinear computational aeroelasticity," Computer Methods in Applied Mechanics and Engineering, vol. 195, no. 17-18, pp. 1973-2001, Mar. 2006. [Online]. Available: http://linkinghub.elsevier.com/retrieve/pii/S0045782505001945

[12] J. Degroote, "Partitioned Simulation of Fluid-Structure Interaction," Archives of Computational Methods in Engineering, vol. 20, no. 3, pp. 185-238, Jul. 2013. [Online]. Available: http://link.springer.com/10.1007/s11831-013-9085-5

[13] X. Zhao, Y. Zhu, and S. Zhang, "Transonic wing flutter predictions by a loosely-coupled method," Computers \& Fluids, vol. 58, pp. 45-62, Apr. 2012. [Online]. Available: http://linkinghub.elsevier.com/retrieve/pii/S0045793012000035

[14] R. Zwaan and B. Prananta, "Fluid/structure interaction in numerical aeroelastic simulation," International Journal of Non-Linear Mechanics, vol. 37, no. 4-5, pp. 987-1002, Jun. 2002. [Online]. Available: http://linkinghub.elsevier.com/retrieve/pii/S002074620100110X

[15] H. H. Mian, G. Wang, and Z.-Y. Ye, "Numerical investigation of structural geometric nonlinearity effect in high-aspect-ratio wing using CFD/CSD coupled approach," Journal of Fluids and Structures, vol. 49, pp. 186-201, Aug. 2014. [Online]. Available: http://linkinghub.elsevier.com/retrieve/pii/S0889974614000875

[16] J. Joo, C. Marks, and L. Zeintarski, “AFRL Variable Camber Compliant Wing - Wind Tunnel Testing," in SciTech 2015, Kissimmee, FL, 2015.

[17] P. R. Spalart and S. R. Allmaras, "A One-Equation Turblence Model for Aerodynamic Flows," Recherche Aerospatiale, vol. 1, pp. 5-21, 1994. 


\section{APPENDIX A}

\section{Additional CFD Results}




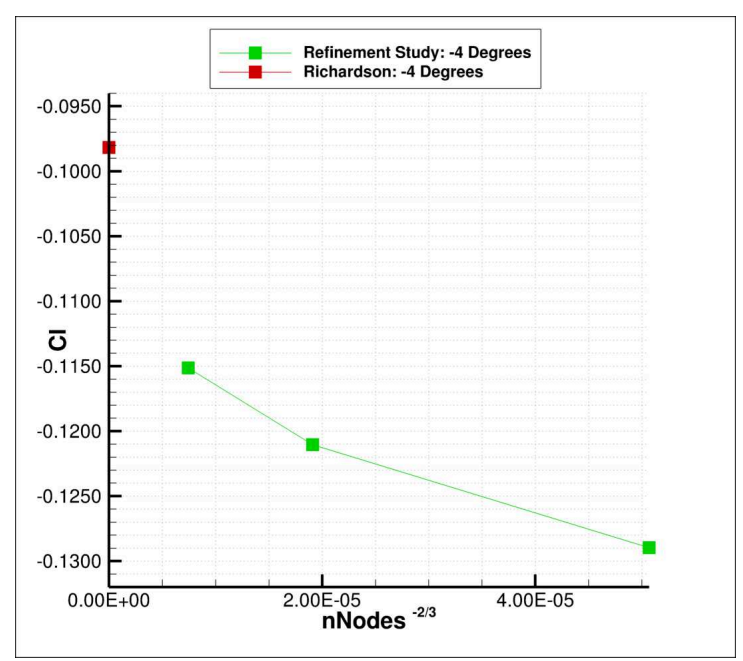

(a) Coeff. of Lift: $-4^{\circ}$

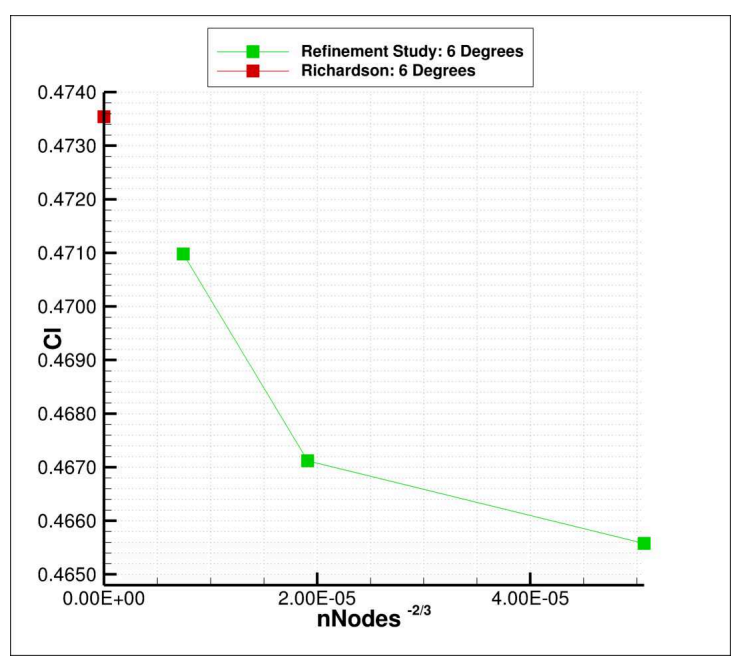

(c) Coeff. of Lift: $6^{\circ}$

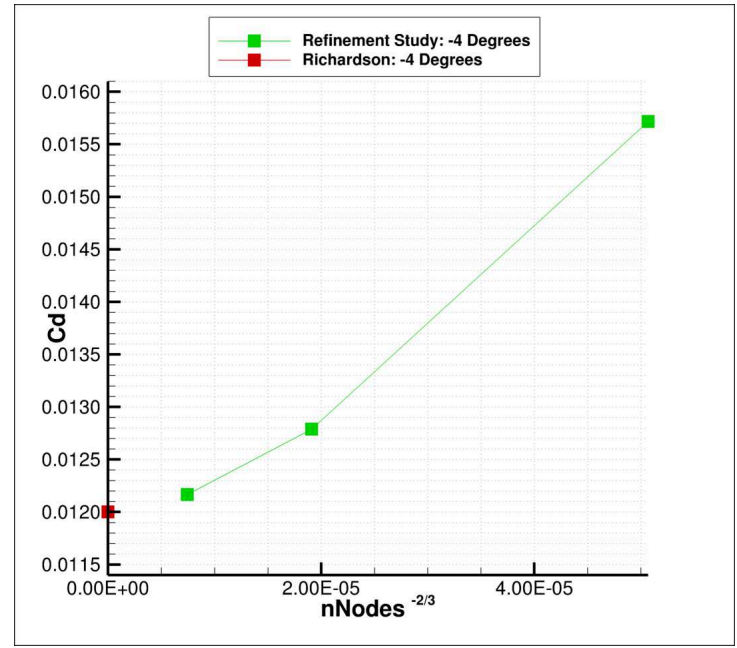

(b) Coeff. of Drag: $-4^{\circ}$

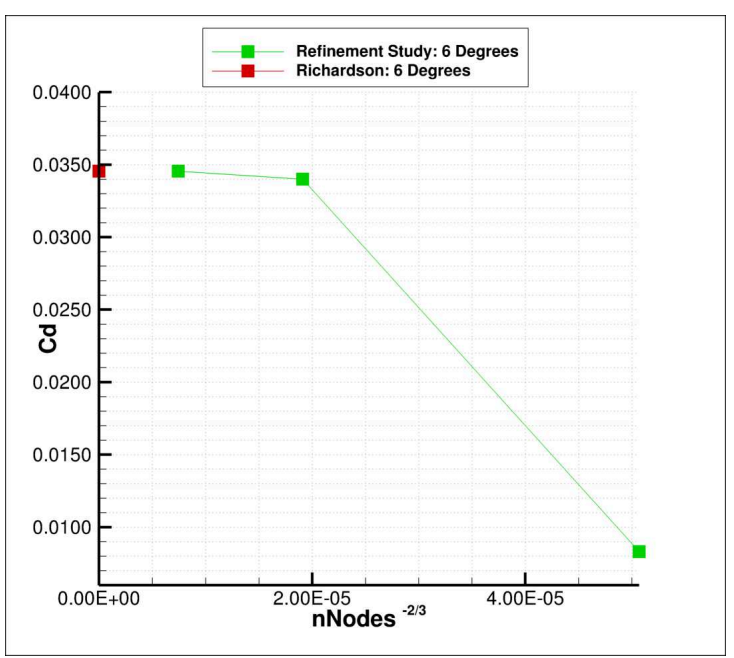

(d) Coeff. of Drag: $6^{\circ}$

Figure A1.1 : CFD Mesh Resolution Study: Initial FSI configuration (NACA 2410) 


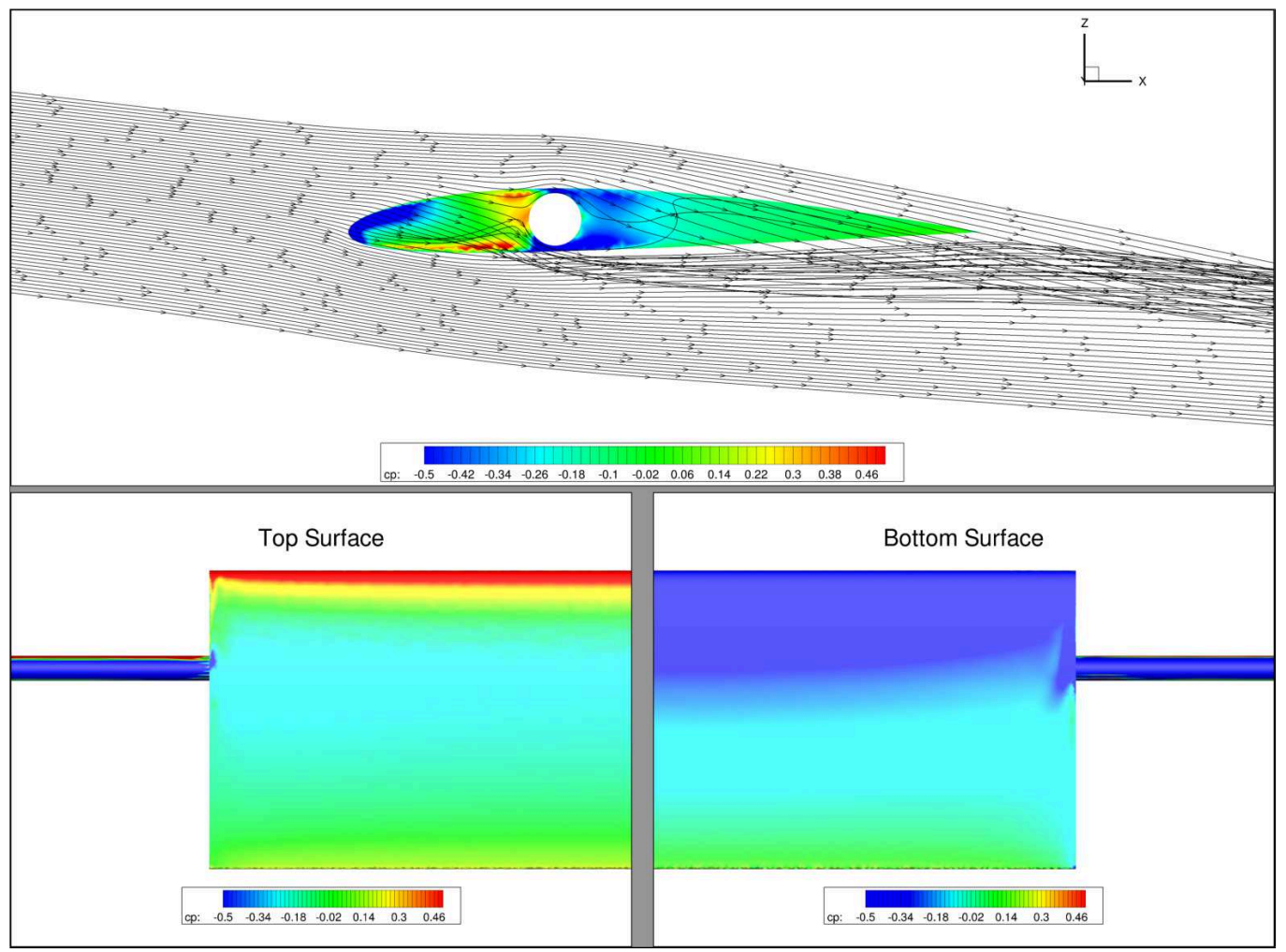

(a) With Support Structure - Alpha: -6 $6^{\circ}$

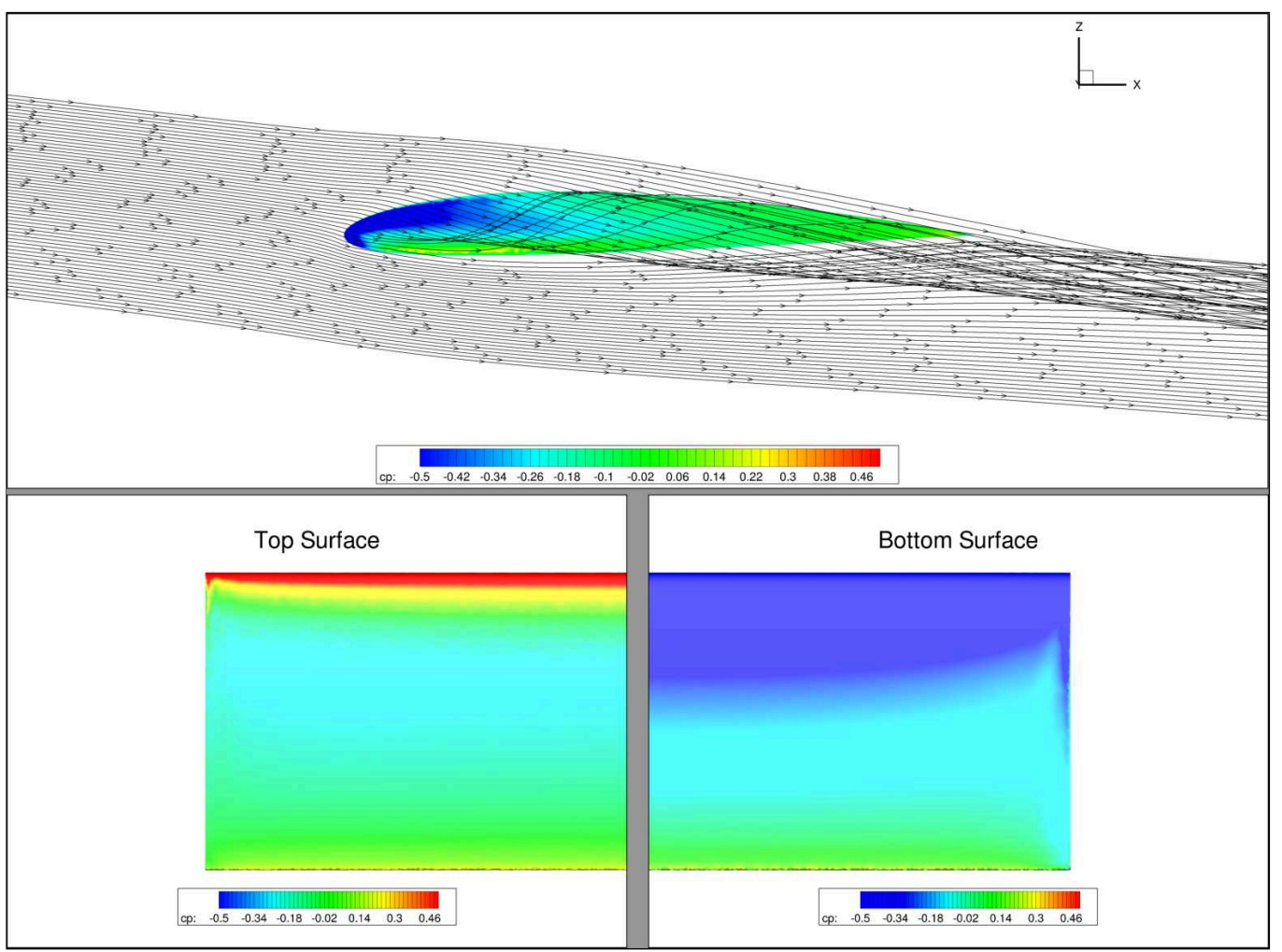

(b) Without Support Structure - Alpha: $-6^{\circ}$

Figure A1.2 : $-6^{\circ}$ Support Structure Interference 


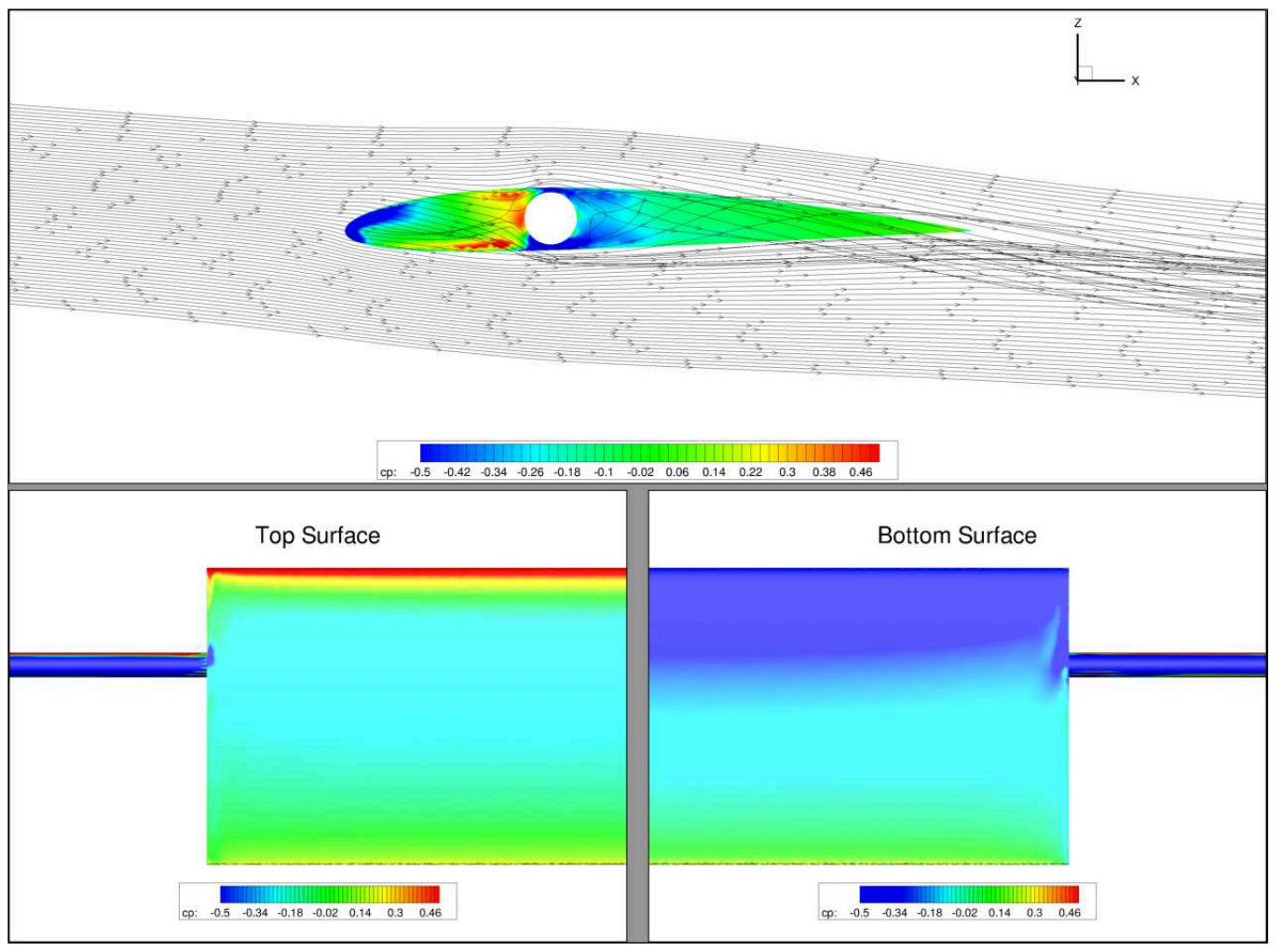

(a) With Support Structure - Alpha: $-4^{\circ}$

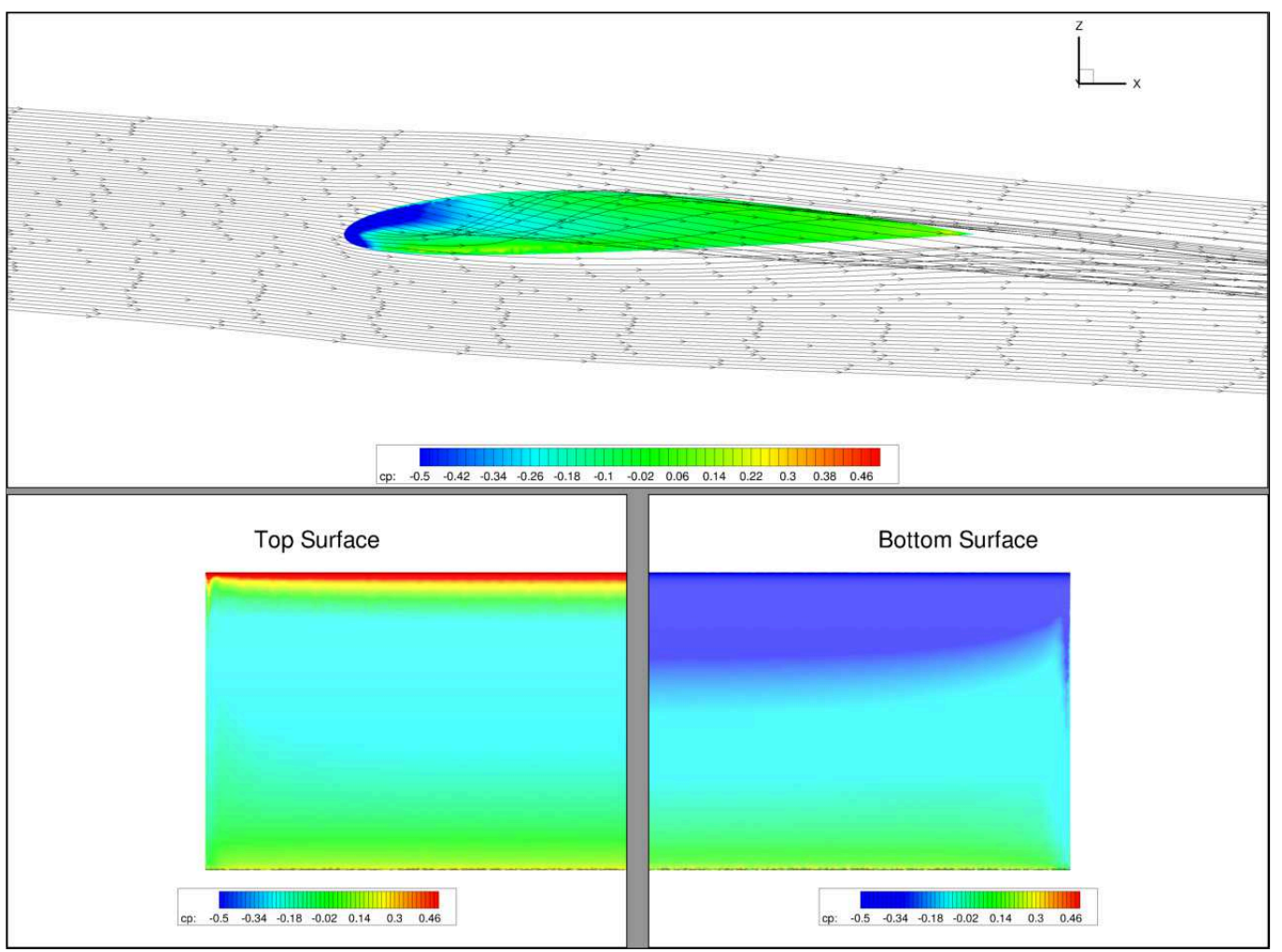

(b) Without Support Structure - Alpha: $-4^{\circ}$

Figure A1.3 : $-4^{\circ}$ Support Structure Interference 


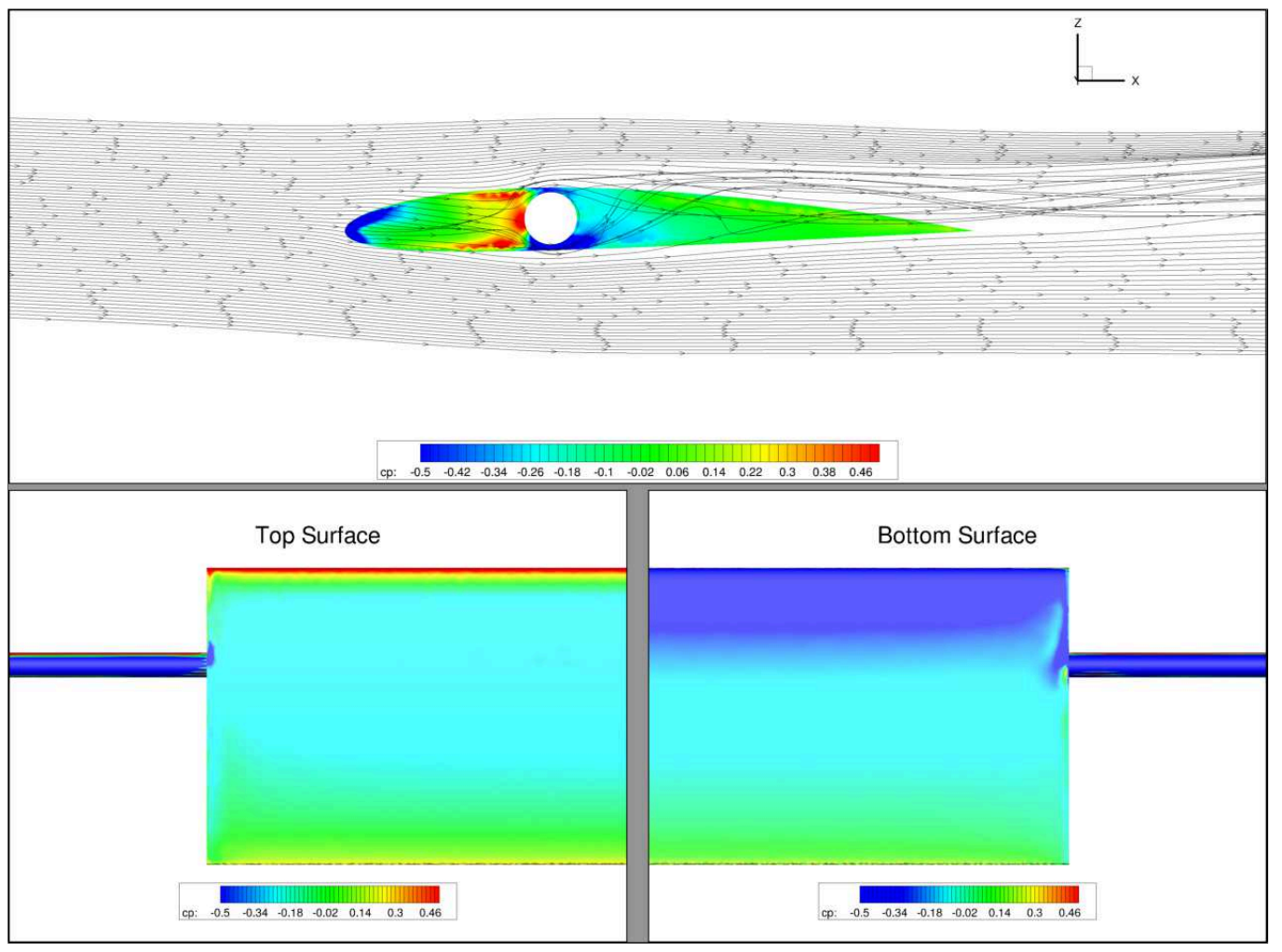

(a) With Support Structure - Alpha: $-2^{\circ}$

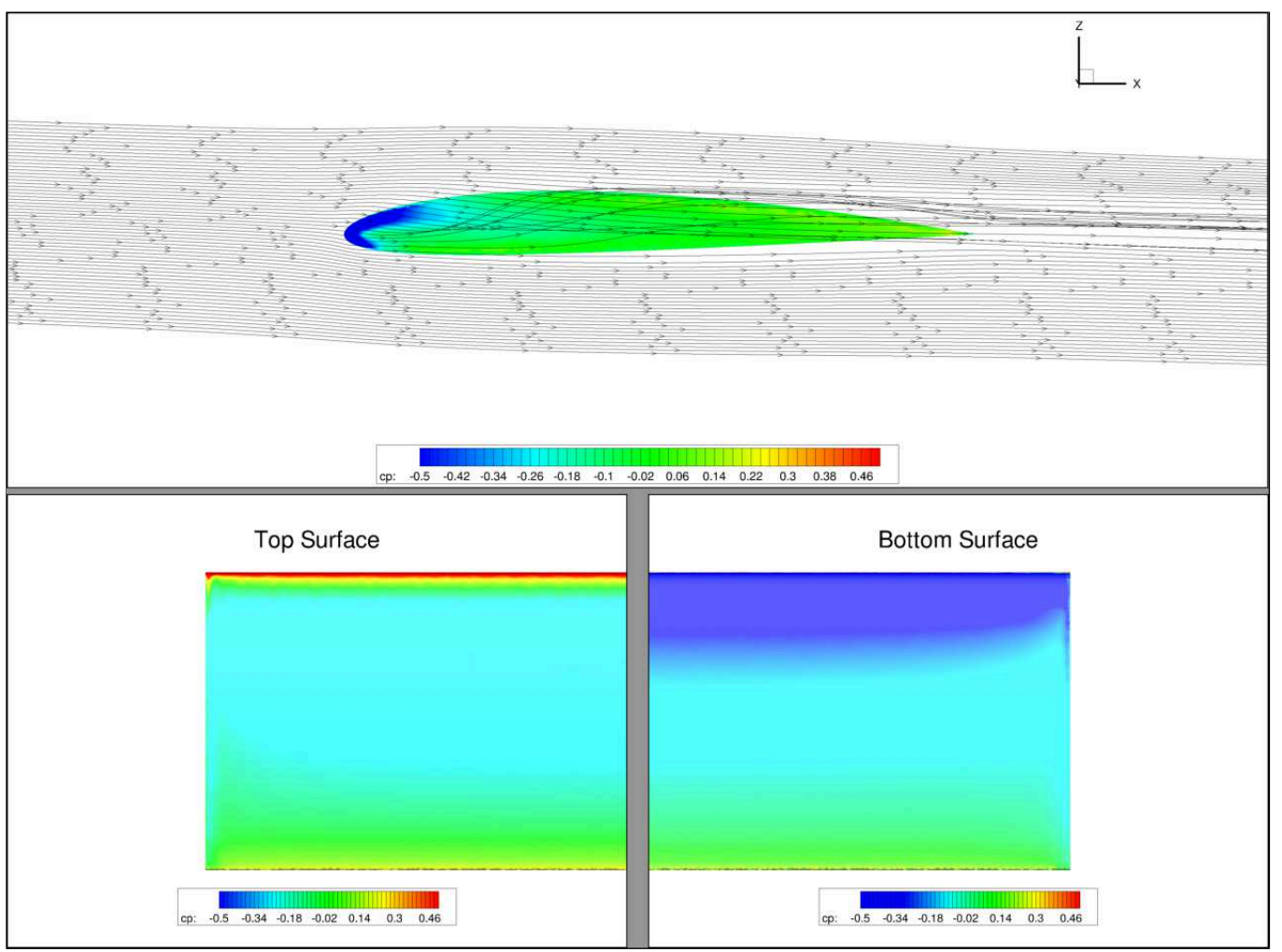

(b) Without Support Structure - Alpha: $-2^{\circ}$

Figure A1.4 : $-2^{\circ}$ Support Structure Interference 


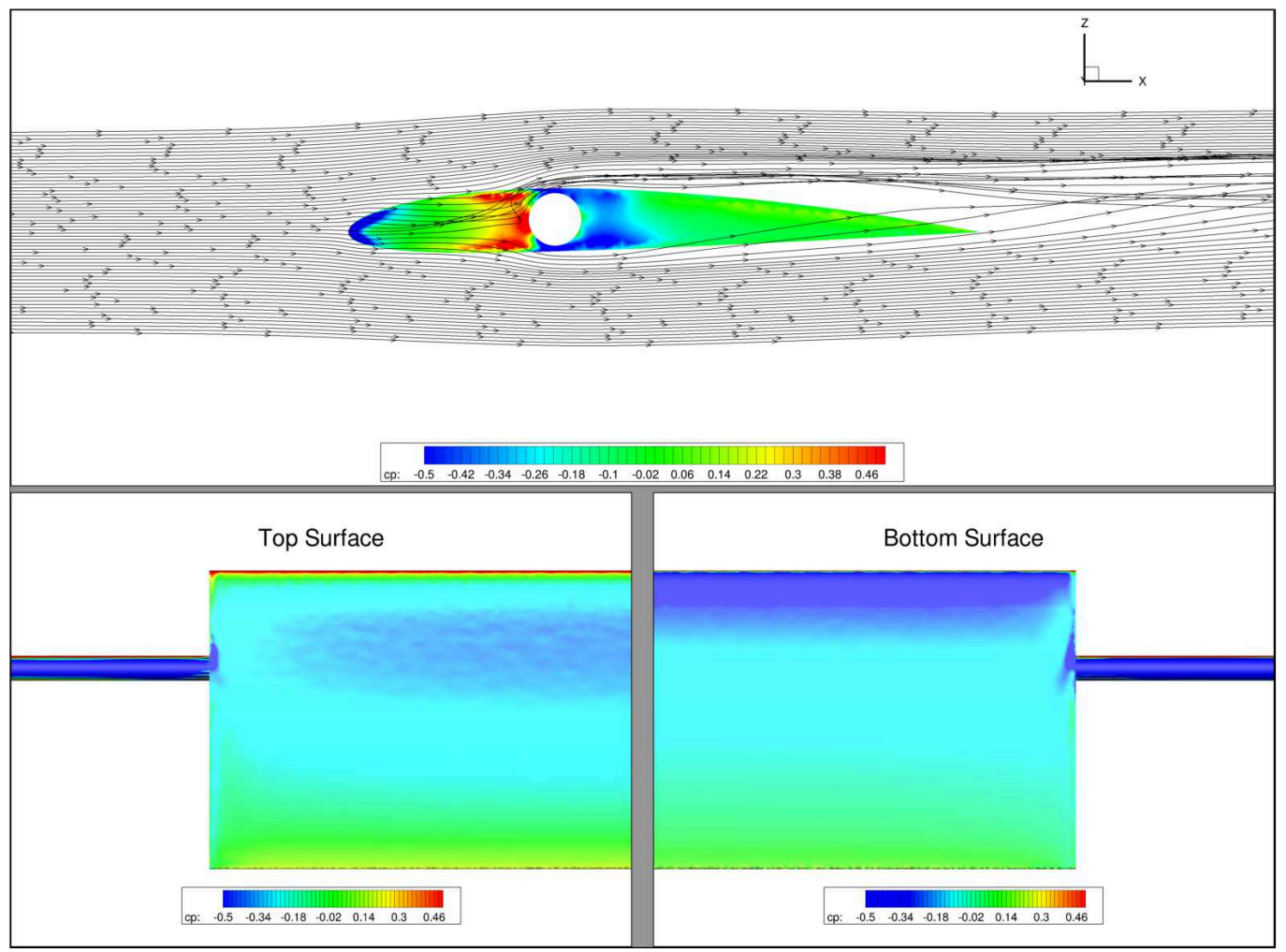

(a) With Support Structure - Alpha: $0^{\circ}$

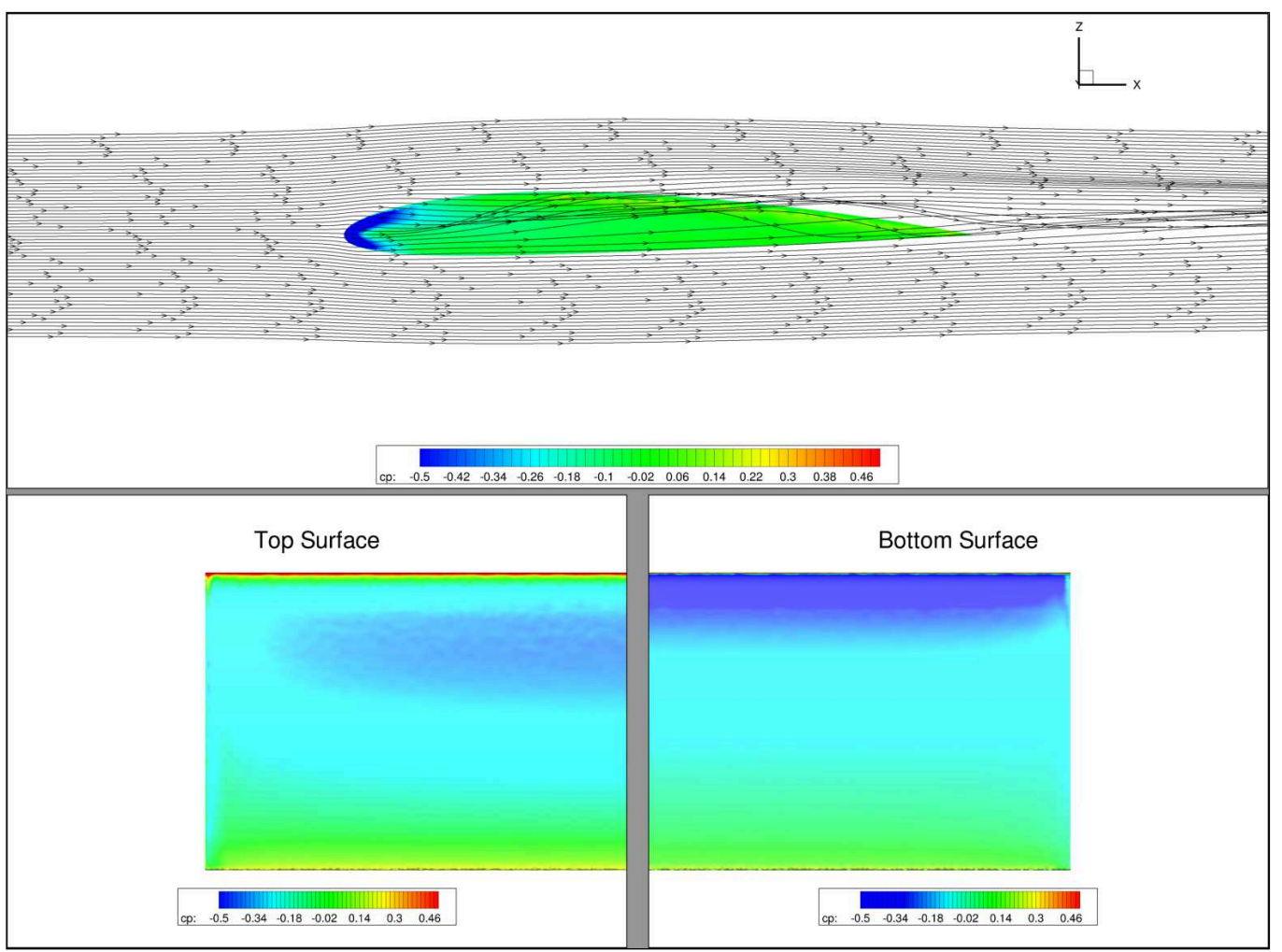

(b) Without Support Structure - Alpha: $0^{\circ}$

Figure A1.5: $0^{\circ}$ Support Structure Interference 


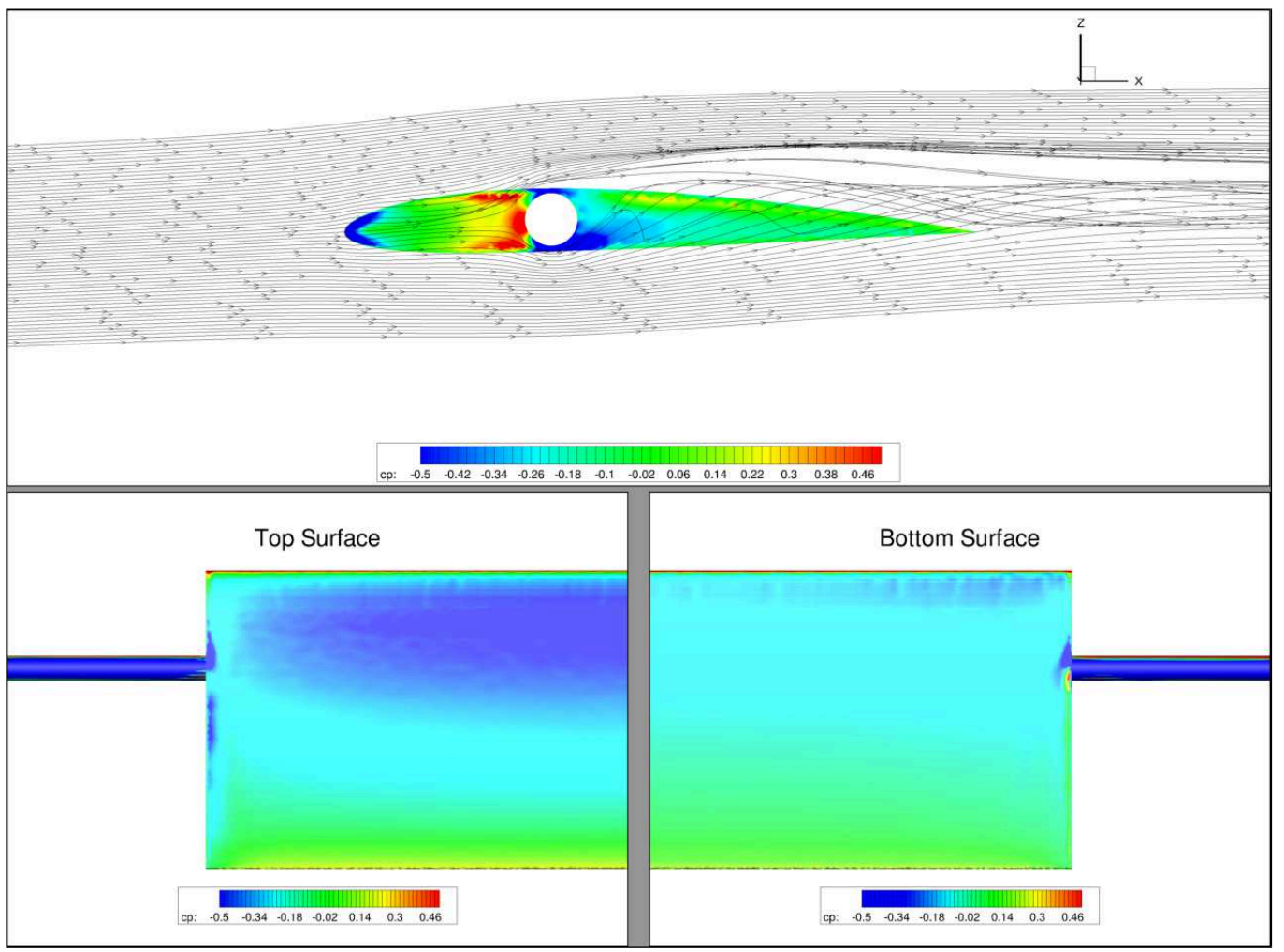

(a) With Support Structure - Alpha: $2^{\circ}$

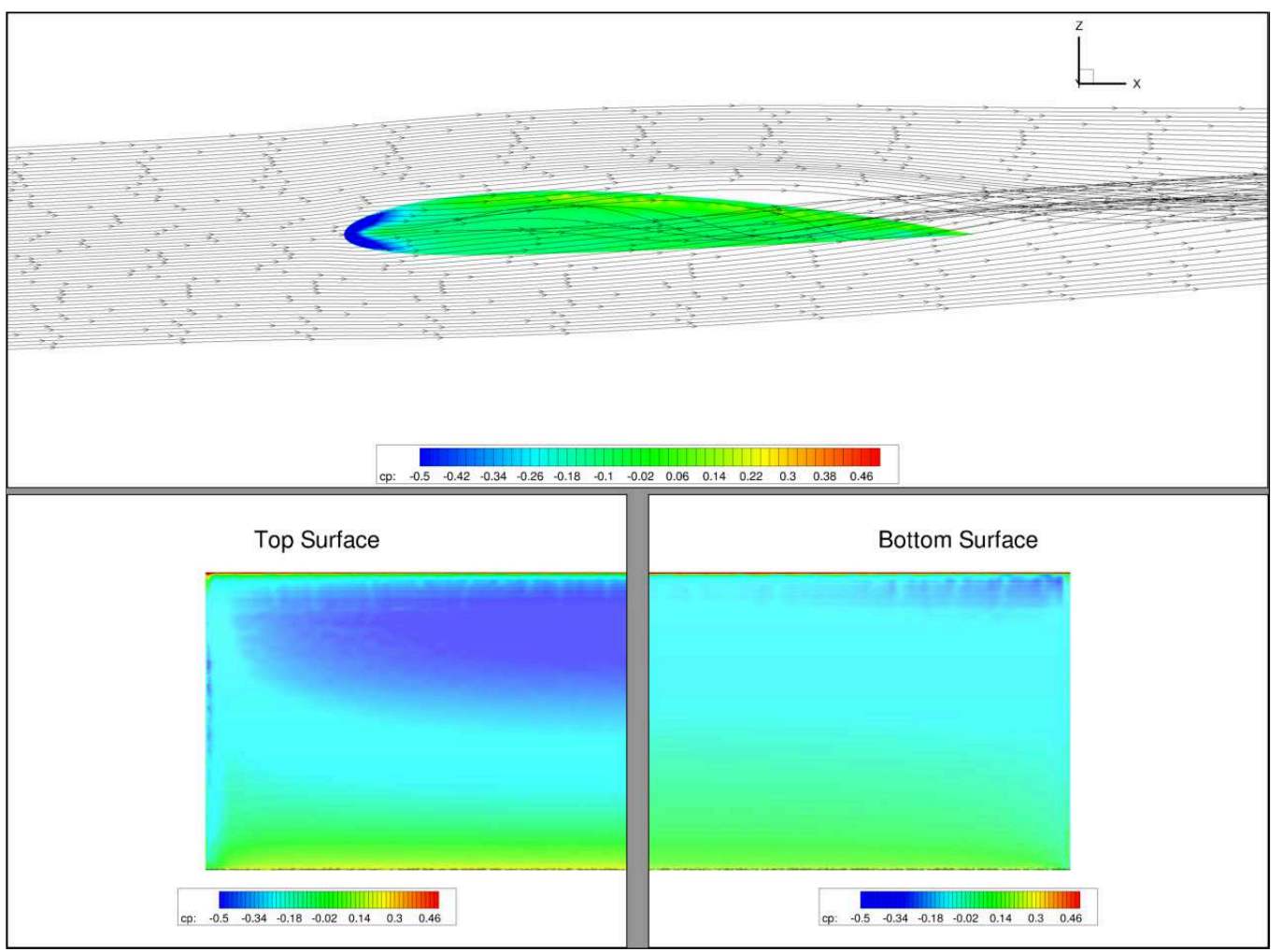

(b) Without Support Structure - Alpha: $2^{\circ}$

Figure A1.6 : $2^{\circ}$ Support Structure Interference 


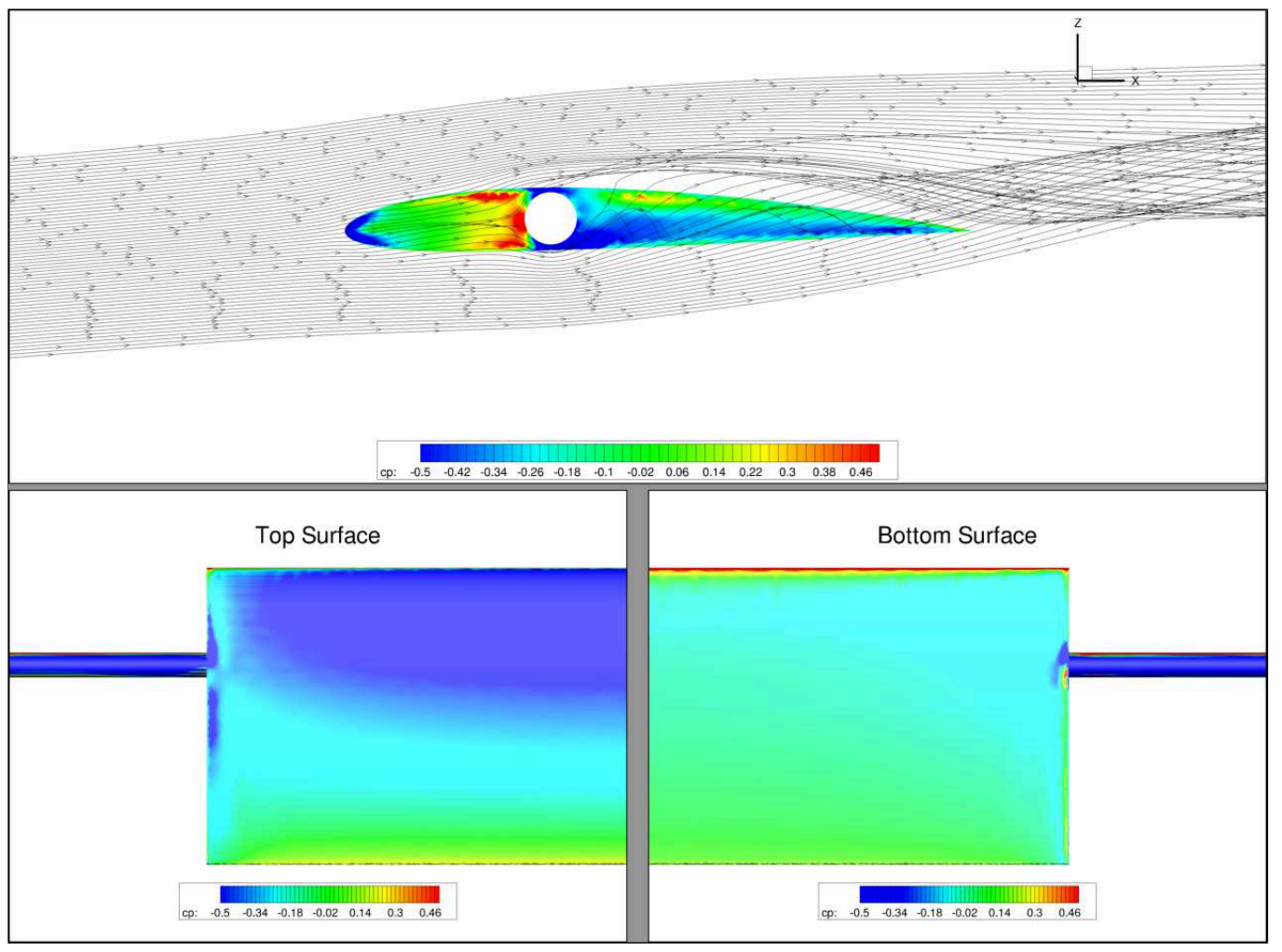

(a) With Support Structure - Alpha: $4^{\circ}$

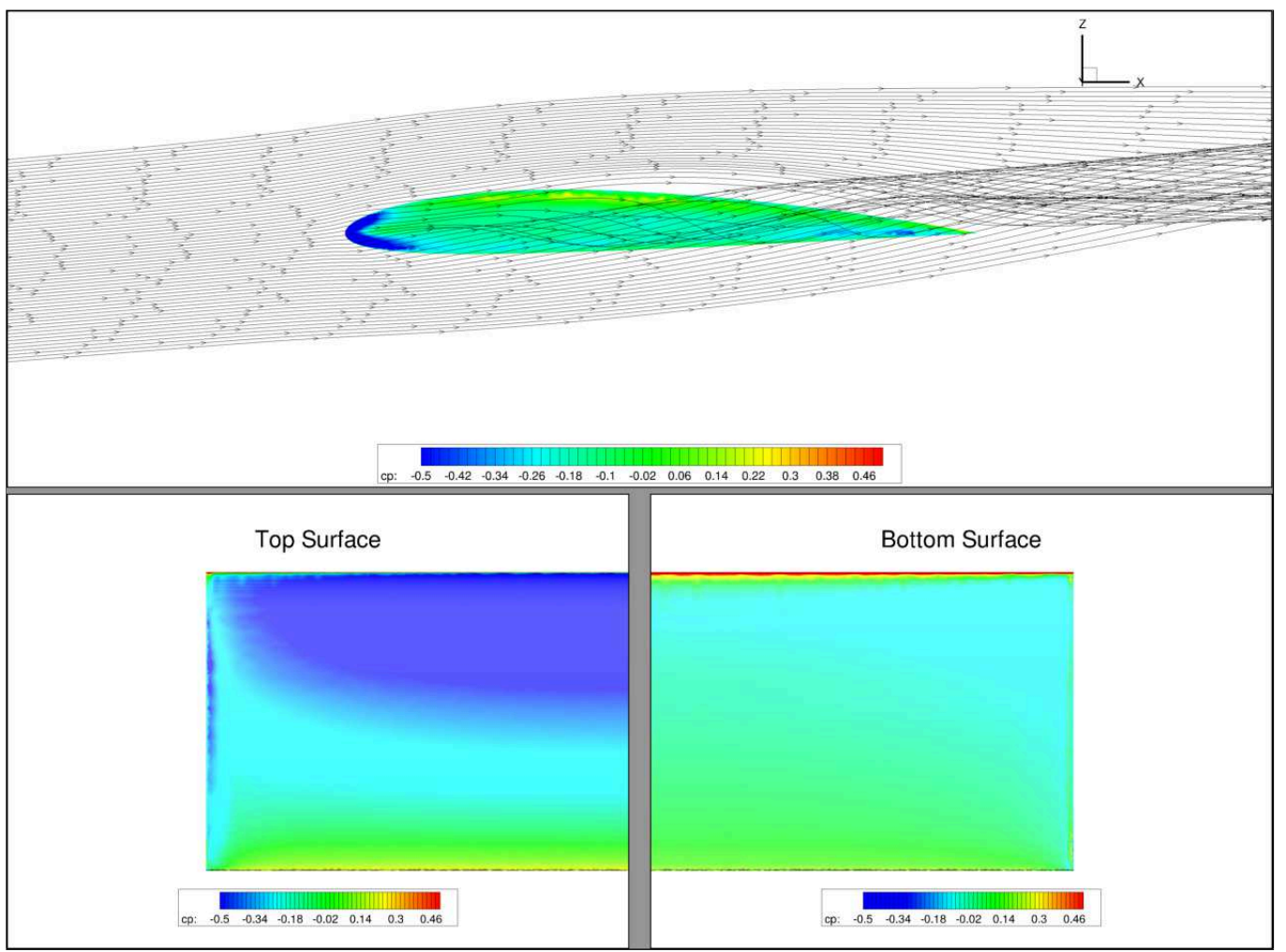

(b) Without Support Structure - Alpha: $4^{\circ}$

Figure A1.7 : $4^{\circ}$ Support Structure Interference 


\section{APPENDIX B}

\section{Additional DIC Results}

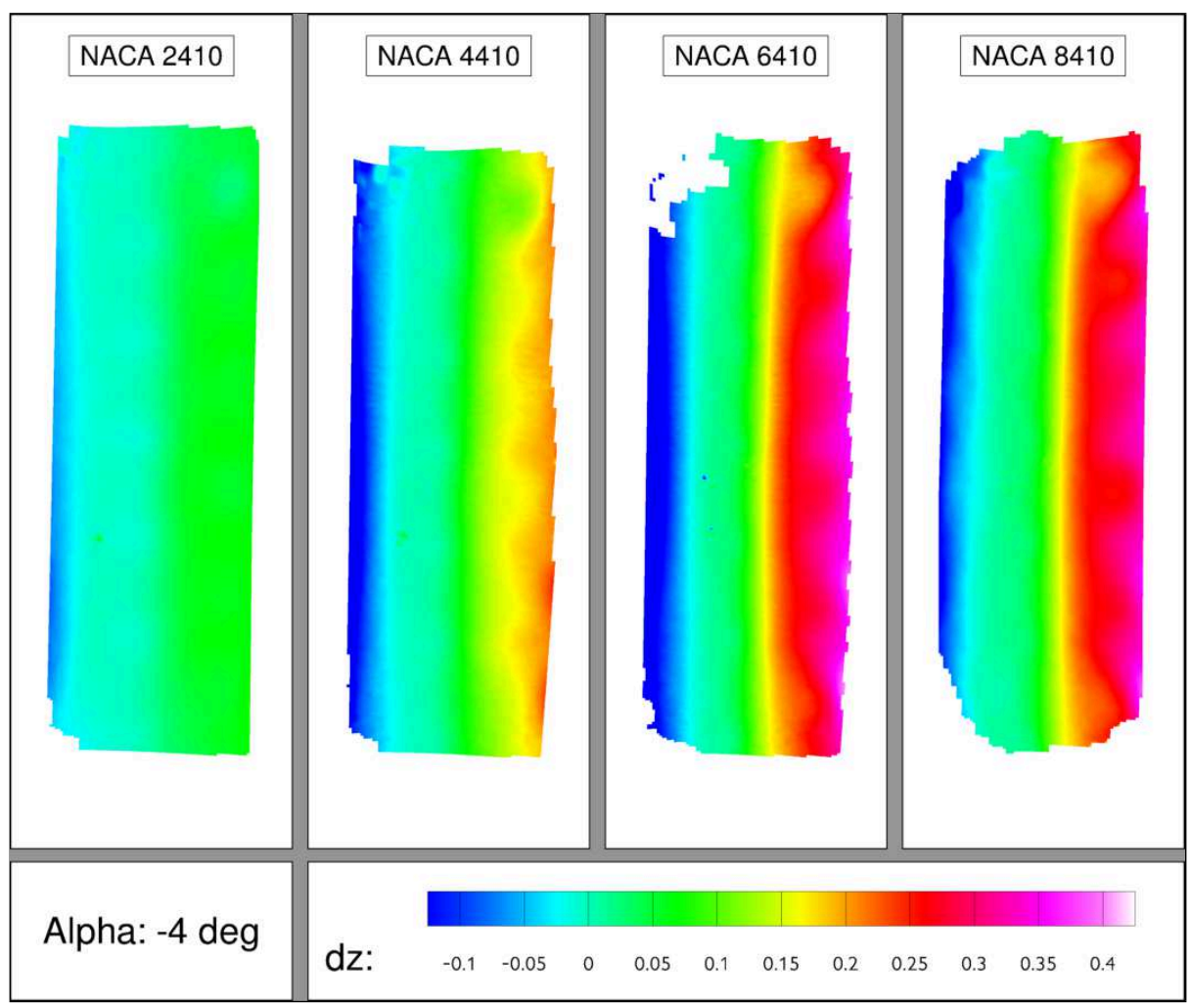

Figure B2.1 : Camber Progression for $-4^{\circ}$ 


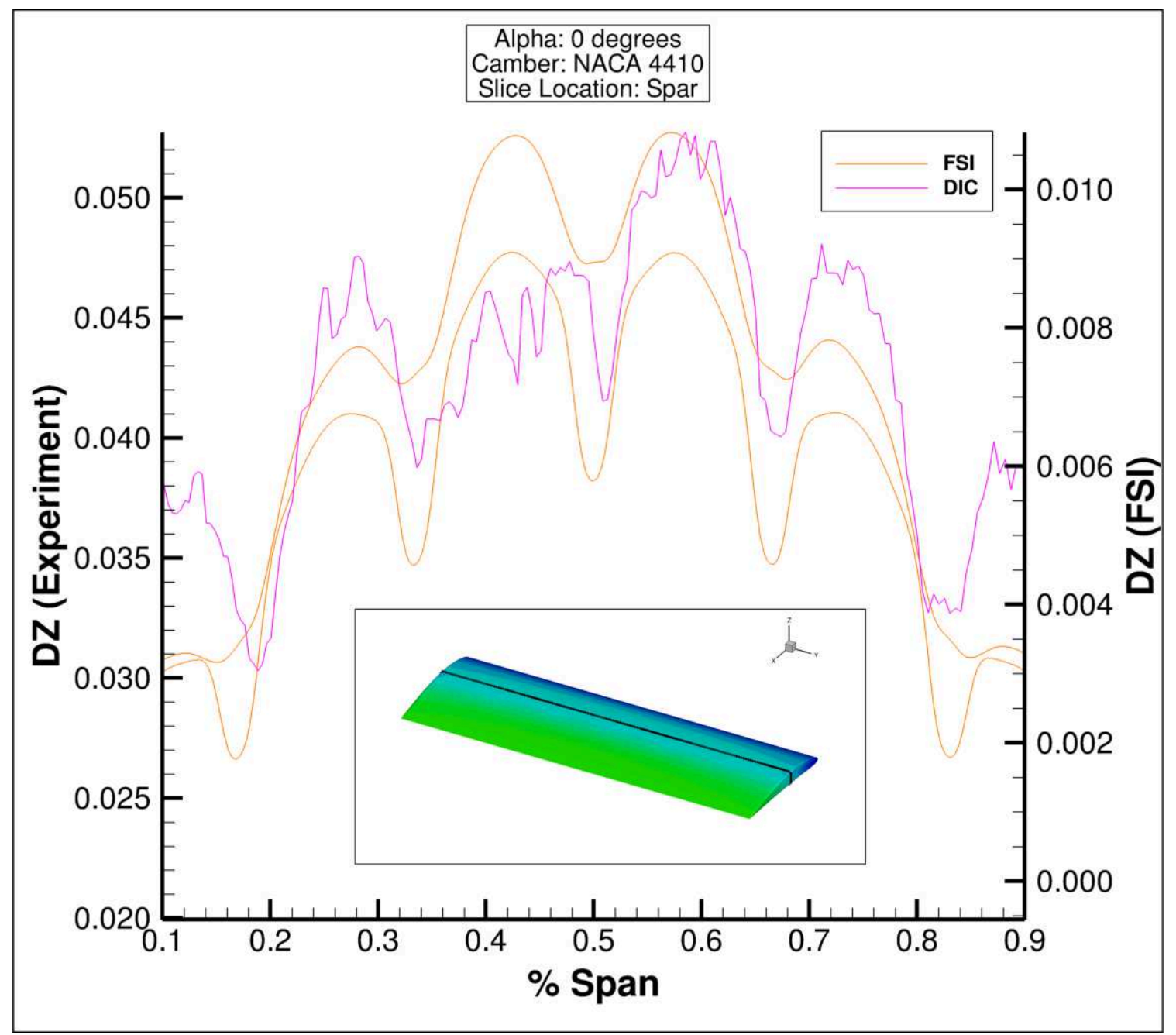

Figure B2.2 : NACA 4410, Alpha $=0$, Span-wise slice at spar 

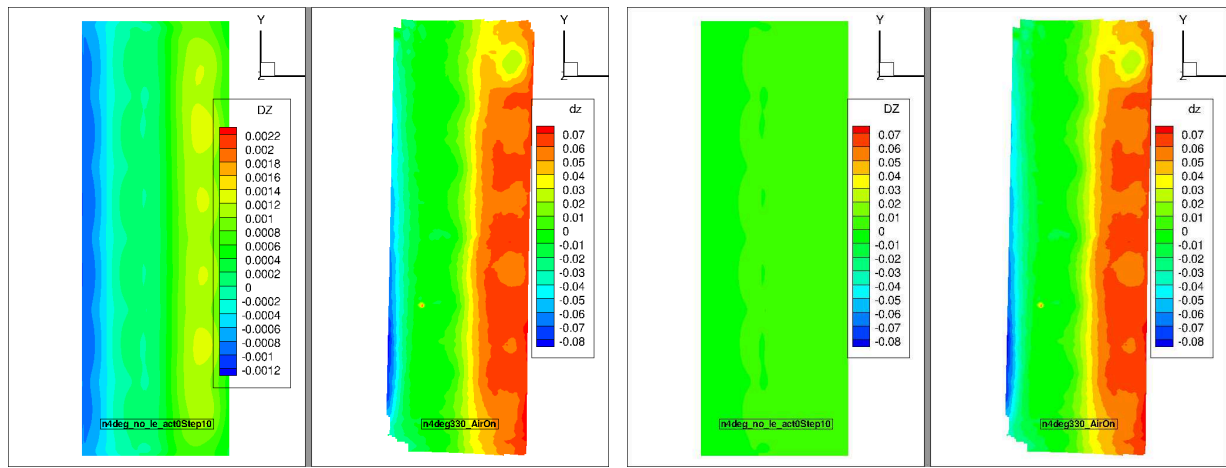

(a) Baseline Resolution: Separate Contour (Left: FSI, Right: DIC)

(b) Baseline Resolution: Matched Contour (Left: FSI, Right: DIC)
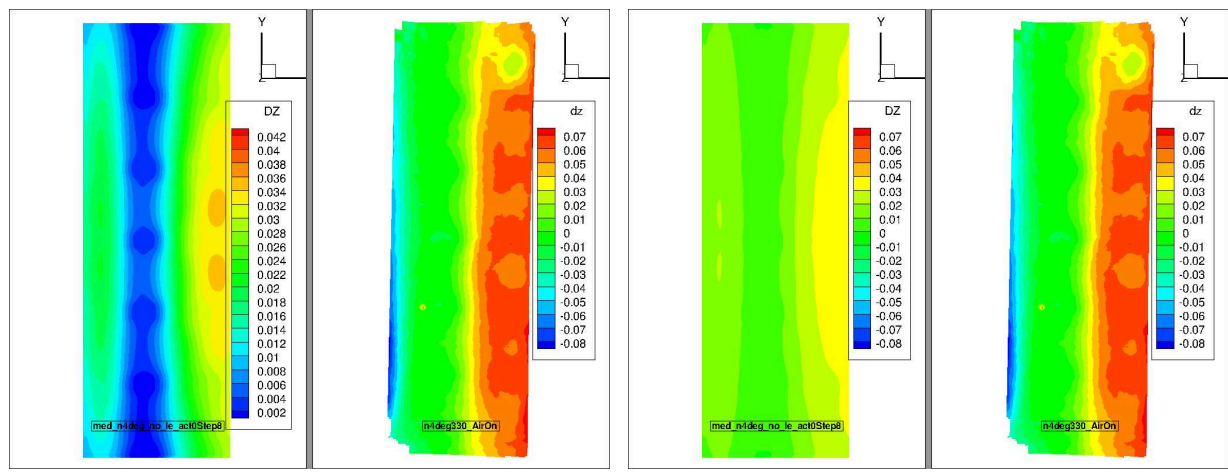

(c) Medium Resolution: Separate Contour (Left: FSI, Right: DIC)

(d) Medium Resolution: Matched Contour (Left: FSI, Right: DIC)

Figure B2.3 : NACA 2410 Target Camber: $-4^{\circ}$ 

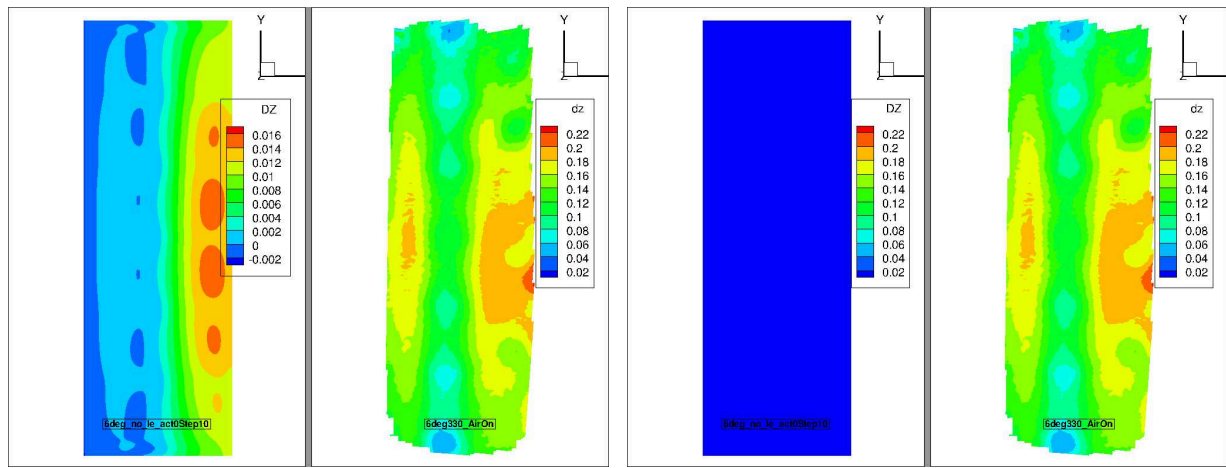

(a) Baseline Resolution: Separate Contour (Left: FSI, Right: DIC)

(b) Baseline Resolution: Matched Contour (Left: FSI, Right: DIC)

Figure B2.4 : NACA 2410 Target Camber: $6^{\circ}$

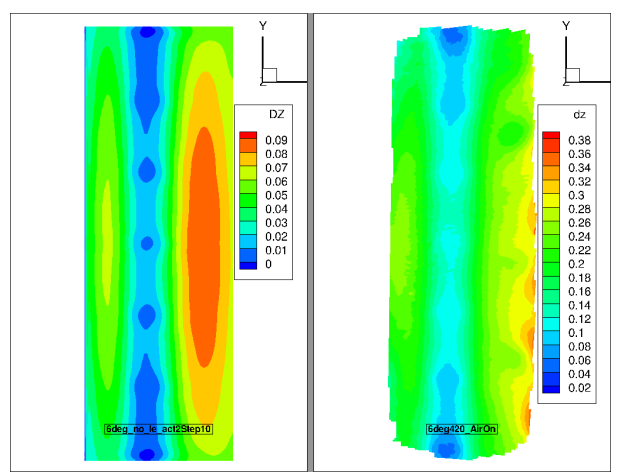

(a) Baseline Resolution: Separate Contour (Left: FSI, Right: DIC)

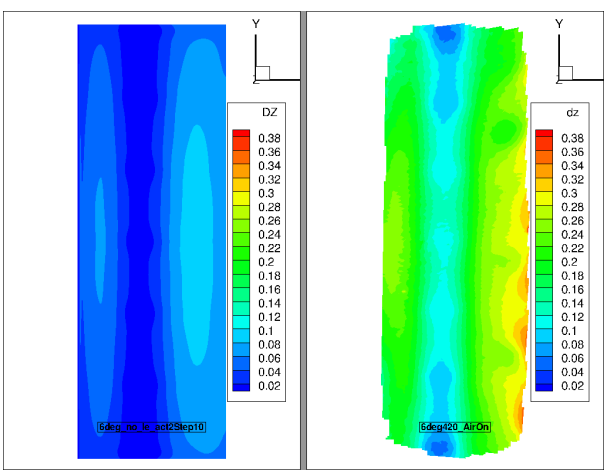

(b) Baseline Resolution: Matched Contour (Left: FSI, Right: DIC)

Figure B2.5 : NACA 4410 Target Camber: $6^{\circ}$ 